Bulletin of the Natural History Museum, 2014, 7: 31-91.

Received 21 May 2014; Accepted 28 Sep 2014.

DOI: $10.5937 / \mathrm{bnhmb} 1407031 \mathrm{~T}$

UDC: 069.51:582.681.26(497.11); 582.681.26.082.5(497.11)

\title{
GENUS VIOLA L. (VIOLACEAE) IN SERBIA \\ - COLLECTIONS OF THE NATURAL HISTORY MUSEUM \\ IN BELGRADE AND THE INSTITUTE OF BOTANY AND \\ BOTANICAL GARDEN “JEVREMOVAC", FACULTY OF BIOLOGY, UNIVERSITY OF BELGRADE -
}

\author{
GORDANA TOMOVIĆ ${ }^{1}$, SNEŽANA VUKOJIČIĆ ${ }^{1}$, KSENIJA MiRJAČIĆ ${ }^{1}$, ANA \\ RADOVIĆ ${ }^{1}$, MARJAN NIKETIĆ ${ }^{2}$ \\ 1'Institute of Botany and Botanical Garden "Jevremovac", Faculty of Biology, \\ University of Belgrade, Belgrade, Takovska 41, 11000 Belgrade, Serbia, \\ e-mail: gtomovic@bio.bg.ac.rs \\ ${ }^{2}$ Natural History Museum, Njegoševa 51, 11000 Belgrade, Serbia, \\ e-mail:mniketic@nhmbeo.rs
}

This paper presents the information on specimens of the genus Viola L. from Serbia deposited in two collections: Herbarium of Natural History Museum in Belgrade (BEO) and the Herbarium of the Institute of Botany and Botanical Garden "Jevremovac", University of Belgrade (BEOU). Overall, 1936 herbarium sheets were examined of which 902 specimens are stored in BEO and 1034 in BEOU. The catalogue of herbarium specimens for 27 plant taxa of the genus Viola from Serbia is presented. Distribution maps for all the investigated species/subspecies are also provided.

Key words: Viola, vascular flora, distribution, Serbia, herbarium collections. 


\section{INTRODUCTION}

Genus Viola L. is the most numerous genus of the family Violaceae with 525-600 species distributed in the most frost-free areas of the world (Clausen 1964, Ballard et al. 1999). In the Flora of Europe, there are 92 Viola species, classified into five sections (Valentine et al. 1968). One of the primary centers of the species diversity in Europe is the Balkan Peninsula, with a large number of endemic taxa (Erben 1985, Stevanović \& Niketić 1990, Mereda et al. 2011).

Diklić $(1972,1977,1986)$ made the last contemporary study of the genus Viola in Serbia and provided basic information about number of species and subspecies present in our country. Of five sections of this genus listed in the Flora Europaea (Valentine et al. 1968), representatives of three sections are present in Serbia. Within the $V$. sect. Viola $(V$. sect. Nominium Ging.), Diklić (1972, 1977) listed 16 native species: $V$. alba Besser, $V$. ambigua Waldst. \& Kit., $V$. canina L., V. elatior Fr., $V$. hirta L., $V$. jordanii Hanry, $V$. mirabilis L., $V$. odorata L., $V$. pumila Chaix, $V$. pyrenaica Ramond, $V$. reichenbachiana Jord. ex Boreau, $V$. riviniana Rchb., $V$. rupestris $\mathrm{F}$. W. Schmidt, $V$. stagnina Kit, $V$. suavis Bieb. and $V$. uliginosa Besser. Only recently Lakušić et al. (2006) reported new adventive species $V$. obliqua Hill for the flora of Serbia. Section Dischidium Ging. is represented by a single species $V$. biflora L. in the flora of Serbia. According to Diklić $(1972,1986)$ and Stevanović \& Niketić (1990), there are 12 species within the $V$. sect. Melanium Ging.: V. aetolica Boiss. \& Heldr., $V$. arvensis Murray, $V$. calcarata subsp. zoysii (Wulfen) Merxm., $V$. dacica Borbás, V. dukadjinica W. Becker \& Košanin, V. elegantula Schott, $V$. grisebachiana Vis., $V$. hymettia, $V$. kitaibeliana Schultes, $V$. orphanidis Boiss., V. speciosa Pant. and V. tricolor L.

However, more than 40 years have passed since the last detailed study of the genus Viola in Serbia was done, and several important papers have appeared in the meantime in botanical literature. The new nomenclature and taxonomic treatments were presented in the floras of several Balkan Peninsula countries (Erben 1985, Raus 1986, Micevski 1995), as well as modern phylogenetic relationships within infrageneric groups in the genus Viola (Ballard et al. 1999, Słomka et al. 2015, etc.).

Therefore, the main aim of this catalogue is to update the list of all species and subspecies of the genus Viola in Serbia, based on inspection and revision of plant specimens from two herbaria: collection of the Natural 
History Museum in Belgrade (BEO) and the Institute of Botany and Botanical Garden „Jevremovac“, Faculty of Biology, University of Belgrade (BEOU). Despite the fact that some basic information about distribution of plant taxa of the genus Viola was provided by Diklić $(1972,1977,1986)$, the total taxa distribution in Serbia has never been treated as a whole before. Therefore, the second objective of this paper is to provide an updated distribution of the species and species of the genus Viola in Serbia, based on herbarium data only. The catalogue of the plant taxa of the genus Viola has been compiled as a contribution to the third volume of the edition „Flora of Serbia“, and may be integrated in the general checklist of Serbian vascular flora as well.

\section{MATERIAL AND METHODS}

Plant material used for the catalogue of plant taxa of the genus Viola from Serbia was deposited in the Herbarium of the Natural History Museum in Belgrade (BEO) and the Herbarium of the Institute of Botany and Botanical Garden "Jevremovac", University of Belgrade (BEOU), (Thiers 2014, http://sweetgum.nybg.org/ih, continuously updated). Within the herbarium BEOU, there are three separate collections: general collection - BEOU, collection of the Department of Plant Ecology and Geography - BEOU-KEGB and the collection of Josif Pančić „Herbarium Pancicianum" - BEOU (Herb Panc).

Identification and revision of the herbarium material of Viola plants were made according to Flora Europaea (Valentine et al. 1968) and the regional Floras relevant for the investigated genus (Grințescu et al. 1955, Diklić 1972, 1977, Delipavlov 1979, Raus 1986, Micevski 1995, Danihelka et al. 2009). The nomenclature follows the Flora Europaea (Valentine et al. 1968), as well as some new data sources like IOPI (http://plantnet. rbgsyd.nsw. gov.au/iopi/iopihome.htm), The Plant List (2013), etc.

The distribution of each Viola species/subspecies in Serbia was presented on the grid map with squares of $10 \mathrm{~km} \times 10 \mathrm{~km}$, based on Military Grid Reference System and the Universal Transverse Mercator (UTM) projection (http://www.luomus.fi/english/botany/afe/map/utm.htm, Lampinen, 2001). On the distribution maps, imprecise records related to wider localities which included two or more UTM $10 \times 10$ squares were marked with sign; doubtful records were presented with a question mark (?). 


\section{RESULTS}

\section{Catalogue of herbarium specimens of the genus Viola from Serbia Viola L.}

\section{V. sect. VIOLA}

Viola alba Besser (Fig. 1)

Banat Bela Crkva: Kusić, UTM EQ36, silikat (leg. Niketić, M., Tomović, G., 15-Apr-2006, det. M. Niketić, BEO); Srem Fruška gora: Andrevlje, UTM CR90, mešovita listopadna šuma (leg. Nikolić, V., Diklić, N., 17-May-1971, det. N. Diklić, 43100 BEO, sub V. suavis, rev. M. Niketić, 25-Sep-2013); Fruška Gora: Vrdnik, UTM DQ09 (leg. Niketić, M., Tomović, G. 30-Mar-2008, det. M. Niketić, BEO); NW Serbia Jablanik: planinarski dom, UTM CP99, Exp. N, livada (leg. Diklić, N., 23-Apr1955, 42953 BEO, sub $V$. odorata, rev. M. Niketić, 25-Sep-2013); (leg. Nikolić, V., Diklić, N. 23-Apr-1955, det. N. Diklić, 42758 BEO); Koceljeva: Jazovnik-Brdarica, Gomilica-kamenolom, UTM DQ03, Exp. S (leg. Niketić, M., Tomović, G. 04-Apr-2004, det. M. Niketić, BEO); Mali Zvornik: Orlovine-Grabovac, UTM CQ51, krečnjak (leg. Niketić, M., Tomović, G. 14-Nov-2010, det. M. Niketić, BEO); Medvednik: južna padina, UTM CP99, Exp. S, bukova šuma (leg. Diklić, N. 26-Jun-1955, det. M. Janković, 42914 BEO, sub V. hirta, rev. M. Niketić, 25-Sep-2013); Mionica: Ribnica klisura, manastir Pastić, UTM DP29, krečnjak (leg. Niketić, M., Tomović, G. 29-Mar-2008, det. M. Niketić, BEO); Suvobor: Rajac,

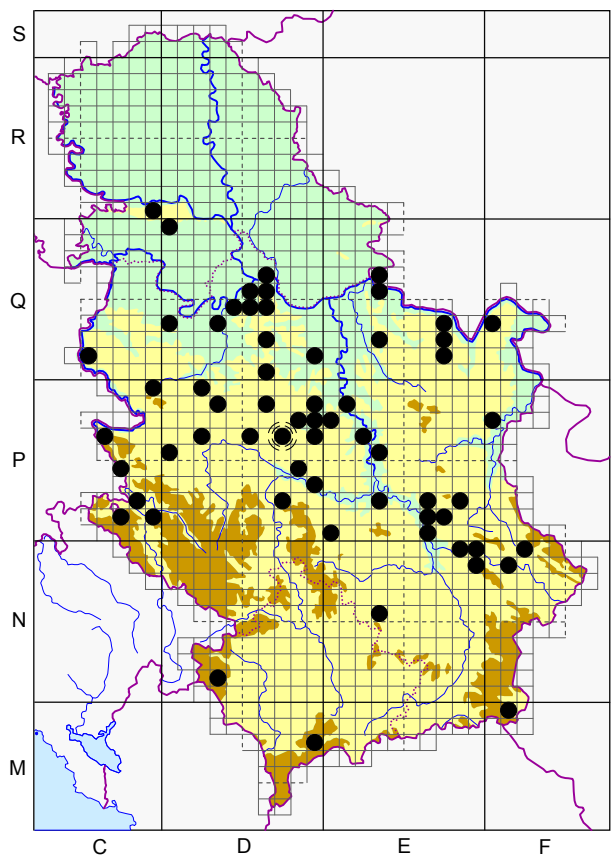

Fig. 1. - Distribution of the species $V$. alba Besser in Serbia.

Slavkovica, UTM DP38 (leg. Stevanović, V. 04-Mar-1974, 45152 BEOU, sub V. sylvestris, rev. M. Niketić, 07-Sep-2013); Šumadija Aranđelovac: Orašac selo, Orašačko brdo, UTM DQ60, silikat (leg. Niketić, M., Tomović, G., 16-Apr-2005, 19416 BEOU-KEGB); Venčac, Brezovac selo, UTM DQ60, serpentinit, kamenjari (leg. Niketić, M., Tomović, G. 21-May-2005, det. M. Niketić, BEO); severne padine, UTM DQ60, Exp. N, 550 m, silikat i mermer (leg. Niketić, M., Tomović, G. 16-Apr-2005, det. M. Niketić, BEO); Beograd: Avala, UTM DQ64, DQ65, borova šuma (leg. Nikolić, v. 14-Apr-1953, det. V. Nikolić, 1559 BEO, sub V. sylvestris, rev. M. Niketić, 25-Sep-2013); Querco-Carpinetum (leg. Lindtner, V. 04-Apr-1954, det. M. Niketić, 1565 BEO, sub Viola sp.); smrčeva šuma (leg. Nikolić, V. 01Apr-1953, det. V. Nikolić, 1545 BEO, sub V. odorata, rev. M. Niketić, 25-Sep-2013); (leg. Lindtner, V. 04-Apr-1954, det. V. Lindtner, 1535 BEO); (leg. Mišić, V. 15-May-1952, det. M. Niketić, 40767 BEOU, sub Viola sp.); (leg. Stevanović, V. 15-Mar-1972, det. M. Niketić, 4536 BEOU-KEGB, sub Viola sp.); Beli Potok, UTM DQ65, borova šuma (leg. Nikolić, V. 01-Apr-1953, det. V. Nikolić, 1544 BEO, sub V. odorata, rev. M. Niketić, 25-Sep-2013); Košutnjak, UTM DQ55, šuma (leg. N. 05-Apr-1948, det. P. Černjavski, 1537 BEO); (leg. Černjavski, P. 13-Mar-1927, det. P. Černjavski, 1539 BEO); (leg. Đelii, B. 20Oct-1936, det. P. Černjavski, 1538 BEO); (leg. Janković, M. Apr-1953, det. M. Niketić, 40886 BEOU, sub Viola sp.); (leg. Janković, M., Mešiček 1952, det. M. Niketić, 40863 BEOU, sub Viola sp.); (leg. Lakušić, D. 10-Apr-1986, 950-86 BEOU-KEGB, sub V. hirta, rev. M. Niketić, 07-Sep-2013); (leg. Niketić, M. 08May-2009, det. M. Niketić, BEO); (leg. Rajevski, L. 16-Mar-1938, 1541, 1542 BEO); okolina (leg. 
Černjavski, P. 21-Mar-1926, 40832 BEOU); Resnik, UTM DQ54 (leg. Grebenščikov, O. 15-Apr-1940, 1523 BEO, sub V. hirta, rev. M. Niketić, 25-Sep-2013); Resnik - Ripanj, UTM DQ54, proređena hrastova šuma (leg. Rajevski, L. 09-Mar-1937, det. P. Černjavski, 1540 BEO); Topčider, UTM DQ55 (leg. Pančić, J., det. J. Pančić, 3214 BEOU (Herb. Panc.)); Višnjica, UTM DQ66, Exp. N, šiblje, 23Mar-1938, 1531 BEO); šibljak (leg. Černjavski, P. Apr-1938, 1533 BEO); Gornji Milanovac: Rudnik, Garavica, UTM DP68, silikat (leg. Niketić, M. 09-Jun-2013, det. M. Niketić, BEO); Vujan, Mali Vujan, UTM DP56, šume, Quercetalia pubescentis (leg. Lakušić, D., Vukojičić, S., Jakovljević, K., Kuzmanović, N. 01-Apr-2009, det. M. Niketić, 28824 BEOU-KEGB, sub Viola sp.); Knić, UTM DP76 (leg. Rudski, I. 14Apr-1940, 1302 BEO); Kragujevac: Beloševac, UTM DP96, Quercetum confertae-cerris (leg. Rudski, I. 31-Mar-1940, 8167 BEO); Querco-Carpinetum (leg. Rudski, I. 31-Mar-1940, det. I. Rudski, 8161 BEO); Drača, Bogosavljevica vrh, UTM DP87 (leg. Rudski, I. 22-Apr-1940, det. M. Niketić, 1297 BEO, sub Viola sp.); Komarica-Samar, UTM EP07, DP97, Exp. NE (leg. Rudski, I. 04-Apr-1940, 8168 BEO); Mečkovac, UTM DP97 (leg. Rudski, I. 02-Apr-1940, 8166, 8159 BEO); (leg. Rudski, I. 02Apr-1940, 8162 BEO, rev. M. Niketić, 25-Sep-2013); Poskurice, UTM DP87 (leg. Rudski, I. 26-Mar1940, det. M. Niketić, 1308, 1309 BEO, sub Viola sp.); Resnik, UTM DP98 (leg. Rudski, I. 06-May1939, 8141 BEO, sub V. sepincola, rev. M. Niketić, 25-Sep-2013); Velika jaruga (leg. Rudski, I. 31Mar-1940, det. M. Niketić, 1306 BEO, sub Viola sp.); Obrenovac: Stubline, UTM DQ33, QuercoCarpinetum (leg. Stevanović, V., Jovanović, S., Lakušić, D., Vukojičić, S. 12-Mar-1997, 4888 BEOU-KEGB, sub V. odorata, rev. M. Niketić, 07-Sep-2013); ušće Kolubare, UTM DQ44 (leg. Stevanović, V., Jovanović, S., Lakušić, D., Vukojičić, S. 12-Mar-1997, 4925 BEOU-KEGB); Veliko polje, UTM DQ33 (leg. Stevanović, V., Jovanović, S., Lakušić, D., Vukojičić, S. 12-Mar-1997, 4875 BEOU-KEGB); Veliko polje - Čekićeva šuma, UTM DQ33, Querco-Carpinetum (leg. Stevanović, V., Jovanović, S., Lakušić, D., Vukojičić, S. 12-Mar-1997, 4904 BEOU-KEGB); (leg. Stevanović, V., Jovanović, S., Lakušić, D., Vukojičić, S. 12-Mar-1997, 4867 BEOU-KEGB, sub V. odorata, rev. M. Niketić, 07-Sep-2013); Sopot: Rogača, UTM DQ62, 100 m, kompozitna podloga, šumarak i potok pored puta (leg. Niketić, M., Tomović, G. 16Apr-2005, det. M. Niketić, BEO); Pomoravlje Braničevo: Tribrode, Petlovac, UTM EQ35, 250 $\mathrm{m}$, kompozitna podloga, stepski fragmenti (leg. Niketić, M., 16-Feb-2014, det. M. Niketić, BEO); Jagodina, UTM EP26, po branicima (leg. Pančić, J. 01-Apr-1847, det. J. Pančić, 3263 BEOU (Herb. Panc.), sub V. odorata, rev. M. Niketić, 07-Sep-2013); Paraćin: Glavica, UTM EP35, 150-250 m, silikat (leg. Niketić, M., Tomović, G. 24-Apr-2005, det. M. Niketić, BEO); Požarevac: Stig, Kula, UTM EQ32, silikat (leg. Niketić, M., Tomović, G. 26-Jan-2008, det. M. Niketić, BEO); Smederevska Palanka (leg. Pečnik 1943, det. P. Černjavski, 8174 BEO, sub V. hirta, rev. M. Niketić, 25-Sep-2013); Glibovac, UTM DQ91, šuma (leg. Lindtner, V. 02-Apr-1950, det. M. Niketić, 1261 BEO, sub Viola sp.); (leg. Tomić, A. 24-Mar-1992, det. A. Tomić, 41072 BEOU, sub V. hirta, rev. M. Niketić, 07-Sep-2013); Velika Morava: Bagrdan klisura, Bagrdan, UTM EP18, silikat, Quercetum frainetto-cerris (leg. Niketić, M., Tomović, G. 17-Mar-2007, det. M. Niketić, BEO); manastir Janković, UTM EP18, silikat, Quercetum frainetto-cerris (leg. Niketić, M., Tomović, G. 17-Mar-2007, det. M. Niketić, BEO); manastir Tomić, UTM EP18, silikat, Quercetum frainetto-cerris (leg. Niketić, M., Tomović, G. 17-Mar-2007, det. M. Niketić, BEO); NE Serbia Đerdapska klisura: Pesača potok, UTM EQ73, bukova šuma (leg. Nikolić, V., Diklić, N., 29-Jun-1967, det. N. Diklić, 42760 BEO); Štrbac, Veliki Štrbac, UTM FQ03, 600 m, mešovita šuma (leg. Diklić, N. 10-Oct-1963, 42913 BEO, sub V. hirta, rev. M. Niketić, 25-Sep-2013); Majdanpek: Debeli lug, Veliki Pek - klisura, UTM EQ71, krečnjak (leg. Niketić, M. 08-Apr-2010, det. M. Niketić, BEO); Majdanpečka Domena, UTM EQ71, Jul-1947, 8175 BEO, rev. M. Niketić, 25-Sep-2013); greben Konstantin, UTM EQ71, 14-Jul-1947, det. N. Diklić, 42916 BEO, rev. M. Niketić, 25-Sep-2013); Jabučin Potok, UTM EQ71 (leg. Sigunov, A. 14-Mar-1948, det. M. Niketić, 1248 BEO, sub Viola sp.); Potkapine, UTM EQ71, bukova šuma (leg. Černjavski, P. 14-Jul-1947, det. N. Diklić, 42755 BEO); Rajkova pećina, UTM EQ72 (leg. Stevanović, V. 25-Apr-1997, 4960 BEOUKEGB, rev. M. Niketić, 21-Sep-2013); Zaječar: Gradskovo, Čokonjarsko brdo, UTM FP07 (leg. Niketić, M., Tomović, G. 01-Jun-2005, det. M. Niketić, BEO); W Serbia Mokra gora (leg. Soška, Th., Jun-1912, det. Th. Soška, 8157 BEO); Ograđenica, UTM CP74, krečnjak (leg. Niketić, M., Tomović, G. 14-Jul-2007, det. M. Niketić, BEO); Tara planina: Derventa reka - kanjon, UTM CP66, krečnjak (leg. Niketić, M. 13-May-1989, det. M. Niketić, BEO, sub V. suavis); Užice: Đetina reka - klisura, Sklopovi, UTM DP05 (leg. Soška, Th. Jun-1926, 45129 BEOU, sub V. pontica, rev. M. Niketić, 07-Sep2013); Zabučje, UTM DP05 (leg. Košanin, N. 09-Jun-1926, 45128 BEOU); Krčagovo, UTM DP05 (leg. Košanin, N. 05-Apr-1889, 40833 BEOU); C Serbia Goč: Gvozdac, Jelen Do, UTM DP26, krečnjak 
(leg. Niketić, M., Tomović, G., 11-Apr-2009, det. M. Niketić, BEO); Gvozdačka (Brezanska) reka, UTM DP72, serpentinit (leg. Niketić, M. 15-Oct-2010, det. M. Niketić, BEO); Kopaonik: Brus, Vlajkovci, UTM EP00 (leg. Popović, I. 06-Sep-1999, 40738 BEOU); Kraljevo: Čukojevac, Vučkovićev zabran, UTM DP84 (leg. Rudski, I. 27-Apr-1940, 1314 BEO); Trstenik: Pasjak, UTM EP32, 300 m (leg. Spasojević J., 14-Mar-1992, det. D. Lakušić, 45080 BEOU, rev. M. Niketić, 07-Sep-2013); Vrnjačka banja: Ljubostinja, UTM DP93 (leg. Pančić, J., 23-Mar-1847, det. J. Pančić, 3227 BEOU (Herb. Panc.), sub V. collina, rev. M. Niketić, 07-Sep-2013); E Serbia Bela Palanka: Čiflik, Ljubatovica, UTM FN18, Exp. S, šljunak (leg. Niketić, M., 18-Apr-2009, det. M. Niketić, BEO); Igrišta, po šumama (leg. Petrović, S. 15-Mar-1879, det. M. Niketić, 41081 BEOU, sub Viola sp., rev. M. Niketić, 07-Sep-2013); Knjaževac: Beli Potok, UTM EP82 (leg. Niketić, M., Tomović, G. 06-Apr-2008, det. M. Niketić, BEO); Niš (leg. Černjavski, P., 43098 BEO, sub V. sepincola, rev. M. Niketić, 25-Sep-2013); Donja Toponica, Popova glava, UTM EP60, silikat (leg. Niketić, M., Tomović, G. 03-Apr-2010, det. M. Niketić, BEO); Jelašnička klisura, Radovanski kamen, UTM EN89, 580 m, krečnjak, Carpinetum orientalis (leg. Niketić, M., Buzurović, U. 07-May-2014, det. M. Niketić, BEO); okolina (leg. Petrović, S. 1879, det. M. Niketić, 45220 BEOU, sub Viola sp.); (leg. Petrović, S., 41053 BEOU, sub V. collina, rev. M. Niketić, 07-Sep-2013); Sićevačka klisura, Oblik, UTM EN99, Carpinetum orientalis (leg. Niketić, M. 03-Mar-2007, det. M. Niketić, BEO); (leg. Niketić, M. 07-Apr-2007, det. M. Niketić, BEO); Niš-Aleksinac: Paligrace, UTM EP61, silikat (leg. Niketić, M., Tomović, G. 01-Apr-2006, det. M. Niketić, BEO); Kremenac, UTM EP61 (leg. Niketić, M., Tomović, G., det. M. Niketić, BEO); Vrelo, UTM EP71, krečnjak (leg. Niketić, M., Tomović, G. 01-Apr-2006, det. M. Niketić, BEO); Vrelska čuka, UTM EP71, krečnjak (leg. Niketić, M., Tomović, G. 01-Apr-2006, det. M. Niketić, BEO); Ozren: Soko Banja, Janior, UTM EP62, pašnjak (leg. Diklić, N. Jun-1960, det. N. Diklić, 42759 BEO); Pirot: Sinjac, UTM FN18, Exp. S, laporac (leg. Niketić, M. 04-Apr-2009, det. M. Niketić, BEO); Temska, UTM FN29, Exp. NW, krečnjak, Querco-Carpinetum (leg. Nikolić, V. 26-Mar-1979, det. M. Janković, 42939 BEO, sub V. odorata, rev. M. Niketić, 25-Sep-2013); Suva planina: Devojački grob, UTM EN98 (leg. Soška, Th. Jun-1912, det. Th. Soška, 8158 BEO); Svrljiške planine: Krupac, istočno od sela, UTM EN99, Exp. N, 400 m, krečnjak, kamenjari i stene (leg. Niketić, M. 01-Mar-2014, det. M. Niketić, BEO); SW Serbia Priboj: Crni vrh, UTM CP82, 750 m, serpentinit (leg. Lindtner, V., 13Apr-1949, det. M. Niketić, 1253 BEO, sub Viola sp.); Oštrik, UTM CP91, 1000-1200 m, krečnjak (leg. Niketić, M., Tomović, G. 14-Aug-2008, det. M. Niketić, BEO); Ožalj, UTM CP71 (leg. Lindtner, V. 14Apr-1949, det. M. Niketić, 1281 BEO, sub Viola sp.); S Serbia Radan: Sokolov vis, Veliki krš, UTM EN35, 1100 m, krečnjak (leg. Niketić, M., Tomović, G., Stevanović, V., Vukojičić, S., 28-Apr-2006, det. M. Niketić, BEO); SE Serbia Dukat planina: Crnook, Jarešnik, UTM FM19, silikat (leg. Niketić, M., 10-Oct-2010, det. M. Niketić, BEO); Kosovo Šar-planina: Ošljak, Gornje selo, UTM DM97 (leg. Rudski, I., 18-Jul-1930, 45071 BEOU, sub V. pontica, rev. M. Niketić, 07-Sep-2013); Metohija Prokletije: Dečanska Bistrica, Dečani, UTM DN31, kestenova šuma (leg. Janković, M., 22-Jul1957, det. M. Niketić, 40889 BEOU, sub Viola sp., rev. M. Niketić, 07-Sep-2013).

Viola ambigua Waldst. \& Kit. (Fig. 2)

Banat Deliblatska peščara (leg. Stevanović, V., 11-Apr-1970, 1294 BEOU-KEGB, sub V. suavis, rev. M. Niketić, 07-Sep-2013); Deliblato, Flamunda, UTM EQ07, pesak, peščara (leg. Soška, Th. 10Jun-1935, 8179 BEO); Zagajičko brdo, UTM EQ17 (leg. Niketić, M., Stevanović, V., Tomović, G., Vukojičić, S. 12-Apr-2009, det. M. Niketić, BEO); Srem Fruška Gora: Stari Slankamen, UTM DQ49 (leg. Stevanović, V., 01-Apr-1980, 1406 BEOU-KEGB, sub V. suavis, rev. M. Niketić, 07-Sep2013); Šumadija Beograd: Makiš, Veliko okno, UTM DQ55 (leg. Soška, Th., 04-Apr-1928, 40881 BEOU, sub $V$. pontica, rev. M. Niketić, 07-Sep-2013); Veliko selo, UTM DQ66 (leg. Vukojičić, S., Tomović, G., Lazarević, P. 03-Apr-2003, det. M. Niketić, 16328 BEOU-KEGB, sub Viola sp.); Višnjica, UTM DQ66, šibljak (leg. Černjavski, P. Apr-1938, det. P. Černjavski, 1505 BEO); (06-Apr-1926, 45155 BEOU); (leg. Bogojević, R., 40757 BEOU, sub V. sylvestris, rev. M. Niketić, 07-Sep-2013); (leg. Lindtner, V. 25-Mar-1934, det. V. Lindtner, 1503 BEO); (leg. Niketić, M. 08-Apr-2006, det. M. Niketić, BEO); (leg. Soška, Th. 03-Mar-1925, 40722, 40723, 40724, 40725 40727, 40728, 40729 BEOU); (Apr-1914, 40730, 40733 BEOU; 40714, 45158 BEOU, sub $V$. hirta $\times$ suavis, rev. M. Niketić, 07-Sep-2013; 40716 BEOU, 


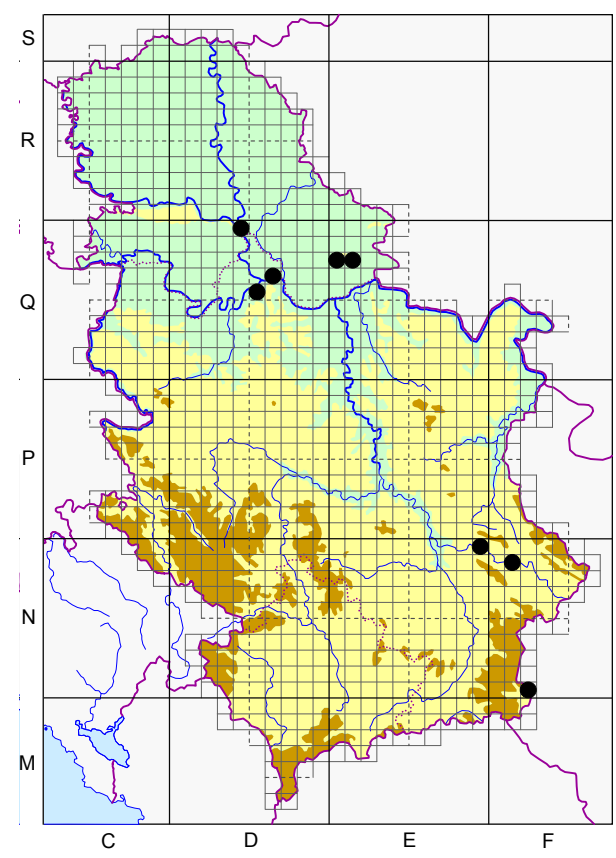

sub V. suavis $\times$ odorata, rev. M. Niketić, 07-Sep2013); (Apr-1928, 45145 BEOU); (09-Mar-1925, 40719, 45130 BEOU); (06-Apr-1926, 40720, 40726 BEOU); Duboki Dol, UTM DQ66 (leg. Soška, Th. 13-Apr-1924, 45113 BEOU; det. M. Niketić, 41058 BEOU); (03-Mar-1925, 40731 BEOU); (01-Apr-1926, 45156 BEOU; 45154 BEOU, sub $V$. hirta, rev. G. Tomović, 07-Sep2013); (06-Apr-1926, 45115 BEOU); (May1927, 45110 BEOU); E Serbia Bela Palanka: Šljivovički vis, UTM FN18, krečnjak (leg. Stevanović, V., Zlatković, B., Tomović, G., Vukojičić, S., Jušković, M., 07-Apr-2004, det. M. Niketić, 22430 BEOU-KEGB, sub Viola sp.); Svrljiške planine: Krupac, istočno od sela iznad kote 289, UTM EN99, Exp. N, 320 $\mathrm{m}$, laporac, kamenjari i stene (leg. Niketić, $M$. 01-Mar-2014, det. M. Niketić, BEO); SE Serbia Rudina planina, UTM FN20, 900-1200 $\mathrm{m}$, krečnjak (leg. Niketić, M., Tomović, G., Zlatković, B., Anačkov, G., 16-Aug-2006, det. M. Niketić, BEO).

Fig. 2. - Distribution of the species

V. ambigua Walds. \& Kit. in Serbia.

Viola canina L. (Fig. 3)

NW Serbia Podrinje: Sokolska planina, Carine, UTM CQ70, krečnjak, livade (leg. Niketić, M., 12-May-2010, det. M. Niketić, BEO); Povlen: Debelo brdo - G. Košlje, UTM CP98 (leg. Stevanović, V., Jovanović, S., Lakušić, D., Pavić, S. 27-May-1994, 1886-940 BEOU-KEGB, sub $V$. sylvestris, rev. M. Niketić, 07-Sep-2013); Šumadija Gornji Milanovac: Zagrađe-Ostrvica, UTM DP59 (leg. Rudski, l., 24-May, det. M. Niketić, 1310 BEO, sub Viola sp.); Ljig: Ba, Čardak, UTM DP39, $500 \mathrm{~m}$, granit (leg. Niketić, M., Jovanović, M. 07-May-2004, det. M. Niketić, BEO); Suvobor: Rajac, Velika provalija, iznad oboda, UTM DP38, 700 m, krečnjak, livade (leg. Niketić, M., Jovanović, M. 07-May-2004, det. M. Niketić, BEO); NE Serbia Bor: Stol, Lučka reka, gornji tok, UTM EP99, livade (leg. Nikolić, V., 23-May-1955, 43104 BEO, sub V. rupestris, rev. M. Niketić, 25-Sep-2013); Veliki krš, Kljancu Mare, UTM EP89 (leg. Pančić, J. 01-Jan-1871, det. M. Niketić, 3185 BEOU (Herb.Panc.), sub Viola sp.); Deli Jovan: Crni vrh, UTM EP99 (leg. Pančić, J. 01-Apr1850, det. J. Pančić, 3223 BEOU (Herb. Panc.)); Majdanpek, UTM EQ71 (leg. Pavlović May-

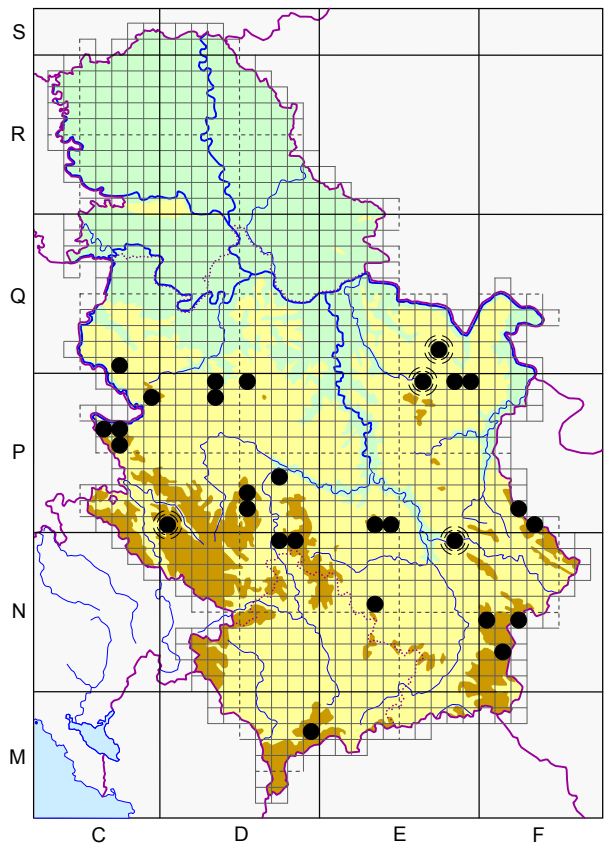

Fig. 3. - Distribution of the species $V$. canina L. in Serbia. 
1878, 40709 BEOU); Stol, UTM EP99, 1100 m, krečnjak, livade (leg. Niketić, M. 08-Jun-1991, det. M. Niketić, BEO); Žagubica, UTM EP69, livada (leg. Blečić, V., 40732 BEOU, sub V. ambigua, rev. M. Niketić, 07-Sep-2013); (leg. Pančić, J. 01-Apr-1876, det. J. Pančić, 3225 BEOU (Herb. Panc.), sub V. canina var. ruppi, rev. M. Niketić, 07-Sep-2013); (leg. Pančić, J., det. J. Pančić, 3279 BEOU (Herb. Panc.), sub V. ruppi, rev. M. Niketić, 07-Sep-2013); W Serbia Čemerno: Ponori, UTM DP52 (leg. Stevanović, V., Niketić, M., Vukojičić, S., Tomović, G., 01-May-2004, det. M. Niketić, 18616 BEOU-KEGB, sub Viola sp.); Nova Varoš, UTM DP00 (leg. Ilić, S. 12-May-1929, 41074 BEOU, sub V. riviniana, rev. M. Niketić, 07-Sep-2013); Tara planina: Karajića bare, UTM CP75, CP76, krečnjak (leg. Stevanović, V., Niketić, M., Tomović, G. 15-Jul-1998, det. M. Niketić, 12775 BEOU-KEGB, sub Viola sp.); Zvezda: Galine, Brusnički potok, UTM CP66, det. M. Niketić, 40791 BEOU, rev. M. Niketić, 07Sep-2013); Podstolac, Štula karaula, UTM CP66 (leg. Pančić, J. 01-May-1880, det. M. Niketić, 3206 BEOU (Herb. Panc.), sub Viola sp.); C Serbia Jastrebac: Veliki Jastrebac, Beli potok, UTM EP30, silikat (leg. Niketić, M., 13-May-2005, det. M. Niketić, BEO); Kopaonik (leg. Pančić, J. 01-May1875, det. J. Pančić, 3224 BEOU (Herb. Panc.), rev. M. Niketić, 07-Sep-2013); Kadijevac, UTM DN79, DN89, 1500 m, livade (leg. Lakušić, D. 07-Jun-1988, det. D. Lakušić, 40799 BEOU, sub V. riviniana, rev. M. Niketić, 07-Sep-2013); Krmeljica, UTM DN79, 1100 m, serpentinit, kitnjakova šuma (Quercetum montanum) (leg. Stevanović, V., Jovanović, S., Lakušić, D. 21-Apr-1990, det. D. Lakušić, 40831 BEOU, sub V. riviniana, rev. M. Niketić, 07-Sep-2013); Kukavica, UTM DN89, 1760 m, serpentinit (leg. Niketić, M., Tomović, G. 27-Jun-2004, 18903 BEOU-KEGB); Kraljevo: Ribnica-Kamenica, Rankovićevo, UTM DP73 (leg. Lindtner, V. 24-Apr-1950, det. M. Niketić, 1259 BEO, sub Viola sp.); Kruševac: Ribarska Banja, okolina, UTM EP40, $600 \mathrm{~m}$, hrastova šuma (leg. Diklić, N. 11-May1959, det. N. Diklić, 42828 BEO); E Serbia Niš, UTM EN89, 1885, 40934 BEOU, rev. M. Niketić, 07-Sep-2013); Ruj planina: Zvonce, UTM FN24, Exp. NW, 1500-1700 m, livade (leg. Nikolić, V., Diklić, N. 18-Jun-1966, det. N. Diklić, 42827 BEO); Stara planina: Babin zub, podnožje, UTM FP30, $1500 \mathrm{~m}$ (leg. Ranđelović, V., Zlatković, B., Vukojičić, S. 08-Jun-1997, 5719 BEOU-KEGB, sub V. sylvestris, rev. M. Niketić, 07-Sep-2013); Hajdučki kamen - Orlove stene, UTM FP21, $1550 \mathrm{~m}$, silikat, obod smrčeve šume (leg. Niketić, M., Jovanović, M. 19-Jul-1998, det. M. Niketić, BEO); Sveti Ni-

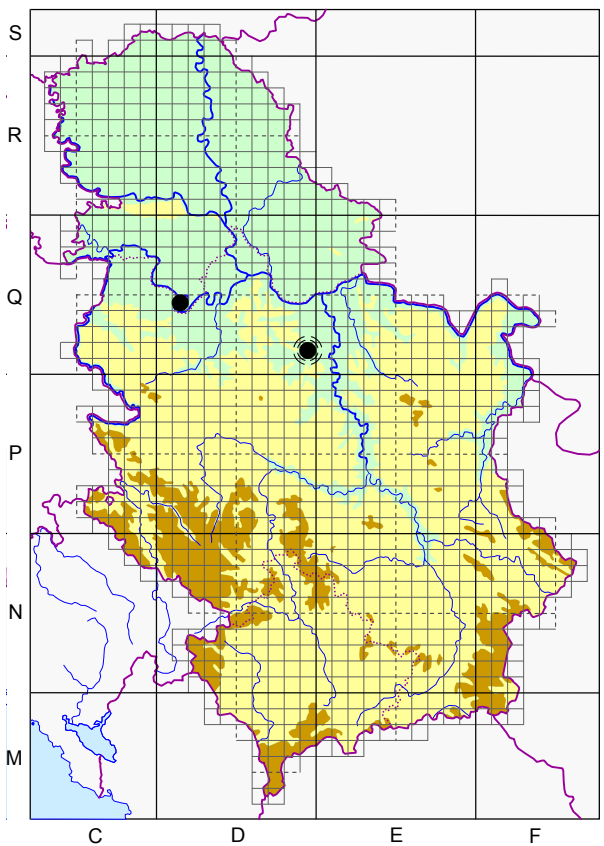

Fig. 4. - Distribution of the species $V$. elatior Fr. in Serbia. kola, Orlova stena (Hajdučki kamen), UTM FP21 (leg. Stevanović, V., Niketić, M., Tomović, G. 18-Jul-1998, det. M. Niketić, 11603 BEOUKEGB, sub Viola sp.); Vojvodin venac, UTM FP30, Exp. W, 1650 m, crveni peščar i konglomerati, stena (leg. Mihailović, T. 15-Jul1993, det. M. Niketić, 40735 BEOU, sub Viola sp., rev. M. Niketić, 07-Sep-2013); Žarkova čuka, UTM FP30, crveni peščar i konglomerati, kraj bukove šume (leg. Mihailović, T. 26-Jul1993, det. T. Mihailović, 40734 BEOU, sub $V$. sylvestris, rev. M. Niketić, 07-Sep-2013); SW Serbia Golija: Crepuljnik, Daićko jezero, UTM DP51 (leg. Kalafatić, V., 24-Jun-1994, det. M. Niketić, 4456 BEOU-KEGB, sub Viola sp.); S Serbia Radan: Sokolov vis, Mali krš, UTM EN35, silikat, stene (leg. Niketić, M., Tomović, G., 01-May-2008, 26866, 26887 BEOUKEGB); SE Serbia Vlasina: Crna Trava, UTM FN04, 45034 BEOU, sub $V$. einseleana, rev. M. Niketić, 07-Sep-2013); (41056, 41057 BEOU, sub $V$. pratensis, rev. M. Niketić, 07Sep-2013); Veliki most, UTM FN12, livada (leg. Černjavski, P., Pavlović, Z., Mišić, V. 23-May1947, det. M. Niketić, 40777 BEOU, sub Viola sp.); stena (leg. Černjavski, P., Pavlović, Z., Mišić, V. 23-May-1947, det. M. Niketić, 40776 BEOU, sub Viola sp.); Kosovo Šar-planina: Ko- 
dža Balkan, Ostrovica, UTM DM97, 1910 m, serpentinit, stene i kamenjari (leg. Lazarević, M., Lazarević, P., 17-Aug-2011, 33152 BEOU-KEGB).

Viola elatior Fr. (Fig. 4)

NW Serbia Vladimirci: Provo, Orlača, UTM DQ14, vlažne šume lužnjaka i zabareni tereni (leg. Diklić, N., 12-May-1988, det. M. Janković, 42852 BEO); Pomoravlje Smederevska Palanka, UTM DQ91, 1959, det. N. Diklić, 42853 BEO); (leg. Pečnik 1943, det. P. Černjavski, 8252 BEO).

Viola hirta L. (Fig. 5)

Bačka Subotičko-Horgoška peščara: Ludaško jezero, Šupljak, zaseok Kosa, UTM DS00, $100 \mathrm{~m}$, aluvijalni nanosi, mezofilne livade (leg. Niketić, M., Tomović, G., 12-Apr2014, det. M. Niketić, BEO); Banat Bela Crkva: Kusić, UTM EQ36, silikat (leg. Niketić, M., Tomović, G., 15-Apr-2006, det. M. Niketić, BEO); Srem Beočin: Veliki čot, UTM CR90, hrastova šuma (leg. Janković, M., 28-Jun1954, det. M. Niketić, 40870 BEOU, sub Viola sp.); Fruška Gora, UTM DR00 (leg. Stevanović, V. 14-Apr-1980, 1436 BEOU-KEGB, sub $V$. suavis, rev. M. Niketić, 07-Sep-2013); NW Serbia Cer planina, UTM CQ74, CQ83, bukova šuma, Apr-1988, det. M. Niketić, 41069 BEOU); Jablanik, UTM CP99, silikat (leg. Pančić, J. 01-May-1880, det. M. Niketić, 3201 BEOU (Herb. Panc.), sub Viola sp.); Krupanj: Kuline, UTM CQ70 (leg. Jurišić, Ž. J. 05-Jun1912, 8173 BEO); Mionica: Ribnica - klisura, manastir Pastić, UTM DP29, krečnjak (leg. Niketić, M., Tomović, G. 29-Mar-2008, det. M. Niketić, BEO); Valjevo, UTM DQ10, 40839 BEOU, rev. M. Niketić, 07-Sep-2013); Šumadija Beograd: Avala, UTM DQ64, DQ65, mešovita šuma (leg. Lindtner, V., 30May-1933, 1522 BEO); Košutnjak, UTM DQ55

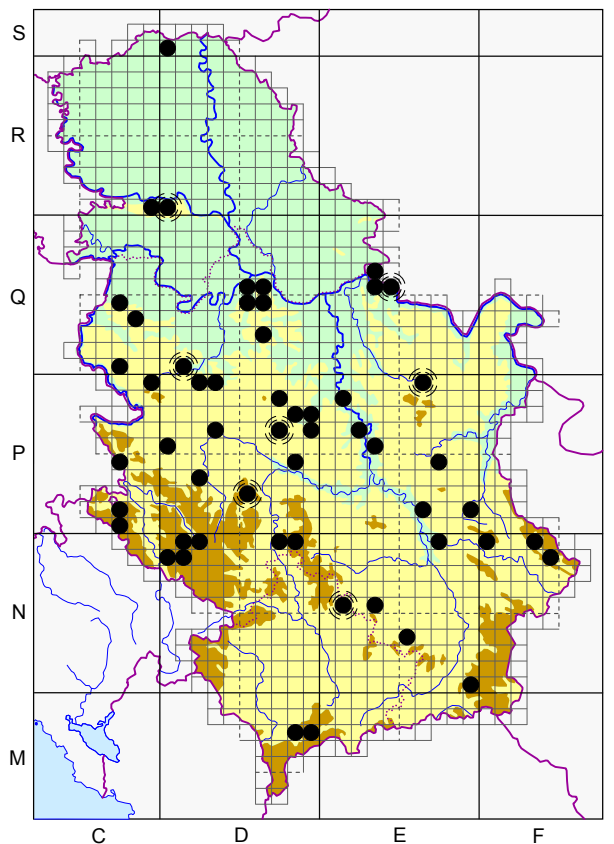

Fig. 5. - Distribution of the species $V$. hirta L. in Serbia.

(leg. Janković, M., Mešiček 1952, det. M. Niketić, 40862 BEOU, sub Viola sp.); (leg. Lindtner, V. May1936, 1511 BEO, sub V. pumila, rev. M. Niketić, 25-Sep-2013); (leg. Pančić, J., det. J. Pančić, 3226 BEOU (Herb. Panc.), sub V. collina, rev. M. Niketić, 07-Sep-2013); Sremčica, Sremački Rt, UTM DQ54, hrastova šuma (leg. Nikolić, V., Pavlović, Z. 23-Apr-1954, det. M. Niketić, 1553, 1556 BEO, sub Viola sp.); Topčider, UTM DQ55 (leg. Pančić, J. 01-Apr-1878, det. J. Pančić, 3237 BEOU (Herb. Panc.)); (leg. Pančić, J., det. J. Pančić, 3233 BEOU (Herb. Panc.)); Gornji Milanovac: Rudnik, Stagari, (Kotraža, Karaula), UTM DP78, $550 \mathrm{~m}$, krečnjak, šikare (leg. Niketić, M., Jovanović, M. 06May-2004, det. M. Niketić, BEO); Knić, UTM DP76, živice (leg. Rudski, I. 14-Apr-1940, 1300 BEO, sub V. pontica, rev. M. Niketić, 25-Sep-2013); (leg. Rudski, I. 14-Apr-1940, det. M. Niketić, 1299 BEO, sub Viola sp.); Kragujevac: Beloševac, UTM DP96, Quercetum confertae-cerris (leg. Rudski, I. 31-Mar1940, 8147 BEO, sub V. sepincola, rev. M. Niketić, 25-Sep-2013); Mečkovac, UTM DP97 (leg. Rudski, I. 02-Apr-1940, 8162 BEO, sub V. alba, rev. M. Niketić, 25-Sep-2013); Petrovac, UTM DP97 (leg. Rudski, I. 07-May-1937, 8229 BEO, sub V. sylvestris, rev. M. Niketić, 25-Sep-2013); Poskurička šuma, UTM DP87, šuma (leg. Rudski, I. 12-Apr-1959, 1277 BEO, rev. M. Niketić, 25-Sep-2013); Šumarice, UTM DP87, 190 m (leg. Marinković, D. 10-Jun-1997, det. V. Stevanović, 40736 BEOU); (leg. Rudski, I. 09-May-1940, 8180 BEO, sub V. ambigua, rev. M. Niketić, 25-Sep-2013); Vitanovac, UTM DP84 (leg. Rudski, I. 07-Apr-1938, det. I. Rudski, 8178 BEO); Ljig: Ba, Čardak, UTM DP39, 500 m, granit (leg. Niketić, M., Jovanović, M. 07-May-2004, det. M. Niketić, BEO); Sopot: Rogača selo, UTM 
DQ62, šumarak i potočić (leg. Niketić, M., Tomović, G. 16-Apr-2005, 19441 BEOU-KEGB); Pomoravlje Jagodina: Đurđevo brdo, UTM EP26 (leg. Pančić, J., 11-Apr-1847, det. J. Pančić, 3235 BEOU (Herb. Panc.)); Paraćin: Glavica, UTM EP35, 150-250 m, silikat (leg. Niketić, M., Tomović, G. 24-Apr2005, det. M. Niketić, BEO); Velika Morava: Bagrdan klisura, Miloševo-Bagrdan, UTM EP18, silikat (leg. Niketić, M. 09-Apr-2010, det. M. Niketić, BEO); Veliko Gradište, UTM EQ45 (leg. Pančić, J., det. J. Pančić, 3234 BEOU (Herb. Panc.)); Zatonjska peščara, UTM EQ35, pesak, peščara (leg. Stevanović, V., Lakušić, D., Vukojičić, S., Tomović, G. 11-Apr-2005, 19351 BEOU-KEGB); NE Serbia Žagubica, UTM EP69 (leg. Pančić, J. 01-Apr-1869, det. J. Pančić, 3236 BEOU (Herb. Panc.)); W Serbia Čemerno, UTM DP52 (leg. Pančić, J., 01-Aug-1860, det. M. Niketić, 3178 BEOU (Herb. Panc.), sub Viola sp.); Ivanjica: Manastir - klisura, UTM DP23, krečnjak (leg. Lakušić, D. 30-Apr1988, 328 BEOU-KEGB); Ovčarsko-Kablarska klisura, UTM DP36 (leg. Soška, Th. Jun-1927, 45135 BEOU, sub V. alba, rev. M. Niketić, 25-Sep-2013); Tara planina: Beli Rzav - klisura, Kršanje, UTM CP74, 500 m, krečnjak, šikare (leg. Niketić, M. 28-May-1989, det. M. Niketić, BEO); Užice: Đetina reka - klisura, Zabučje, UTM DP05 (leg. Košanin, N. 05-Apr-1889, 41014 BEOU, sub $V$. odorata, rev. M. Niketić, 07-Sep-2013); C Serbia Kopaonik: Kaljevac, Šanac, UTM DN79, hrastova šuma (leg. Rajevski, L., 23-Apr-1952, det. L. Rajevski, 40857, 40858 BEOU); Novoselske bačije, Golo Brdo, UTM DN89, $1300 \mathrm{~m}$, granit, kamenjari, sipari i stene (leg. Stevanović, V., Jovanović, S., Lakušić, D. 21-Apr-1990, det. D. Lakušić, 40837 BEOU); E Serbia Niš: Gabrovac, Del - Milljkov del, UTM EN79, 420 m, kompozitna podloga, šikare (leg. Niketić, M., 27Oct-2013, det. M. Niketić, BEO); Markovo kale, UTM EN79, $400 \mathrm{~m}$, kompozitna podloga, šikare (leg. Niketić, M. 12-Apr-2004, det. M. Niketić, BEO); Niš-Aleksinac: Paligrace, UTM EP61, silikat (leg. Niketić, M., Tomović, G. 01-Apr-2006, det. M. Niketić, BEO, sub V. alba); Stara planina: Sirminički vrh, UTM FN39, krečnjak (leg. Niketić, M. 23-Jul-2010, det. M. Niketić, BEO); Visočica klisura, UTM FN48 (leg. Marinković, D. 20-Jul-1991, 2657-91 BEOU-KEGB); Svrljiške planine: Rinjska planina, UTM FN09, 800-1100 m, krečnjak (leg. Niketić, M., Tomović, G. 03-May-2002, det. M. Niketić, 15507 BEOU-KEGB, sub Viola sp.); Rtanj: Kostadinovica, UTM EP74, 700-800 m, krečnjak, šibljak, kamenjari (leg. Niketić, M., Tomović, G., 10-Jul-2009, det. M. Niketić, BEO); Mirovo, Boljevac, UTM EP74 (leg. Soška, Th. Jul-1927, 45127 BEOU); Tresibaba: Šestar, UTM EP91, 780 m, krečnjak, šikare (leg. Niketić, M., Tomović, G. 02-May-2005, det. M. Niketić, BEO); SW Serbia Giljeva: Jelenak, UTM DN18, Exp. NW, 1400-1500 m, krečnjak (leg. Stevanović, V., Niketić, M., Vukojičić, S., Tomović, G., 28-Apr-2006, 20706 BEOU-KEGB); Exp. SW, 1400-1650 m, krečnjak (leg. Niketić, M., Tomović, G., Stevanović, V., Vukojičić, S. 29-Apr-2006, det. M. Niketić, BEO); Krajinovići, Čelo brdo, UTM DN08, Exp. NW, 1000 m, serpentinit (leg. Niketić, M., Tomović, G., Stevanović, V., Vukojičić, S. 28-Apr-2006, det. M. Niketić, BEO); Prijevorac, UTM DN18, Exp. SW, $1250 \mathrm{~m}$, serpentinit (leg. Niketić, M., Tomović, G., Stevanović, V., Vukojičić, S. 24-Apr-2006, det. M. Niketić, BEO); Trijebine, Prijevorac brdo, UTM DN18, $1250 \mathrm{~m}$, serpentinit (leg. Stevanović, V., Niketić, M., Vukojičić, S., Tomović, G. 28-Apr-2006, 20736 BEOU-KEGB); Trijebinska reka - klisura, UTM DN18, $1100 \mathrm{~m}$, krečnjak (leg. Stevanović, V., Niketić, M., Vukojičić, S., Tomović, G. 28-Apr-2006, 20761 BEOU-KEGB); Pešter: Sjenica, Kanjevska reka - klisura, 3 km severno od Sjenice, UTM DN19, Exp. S, 1000 m, krečnjak (leg. Niketić, M., Tomović, G., Stevanović, V., Vukojičić, S. 27-Apr-2006, det. M. Niketić, BEO); (leg. Stevanović, V., Niketić, M., Vukojičić, S., Tomović, G. 27-Apr-2006, 20695 BEOU-KEGB); Stup selo, Vrelo reka - klisura, UTM DN29, 1200 m, krečnjak (leg. Niketić, M., Stevanović, V., Tomović, G., Vukojičić, S. 27-Apr-2006, det. M. Niketić, BEO); Exp. S, 1200 m, krečnjak (leg. Stevanović, V., Niketić, M., Vukojičić, S., Tomović, G. 27-Apr-2006, 20684 BEOU-KEGB); Priboj: Ožalj, UTM CP71, 450 m (leg. Lindtner, V. 14-Apr-1949, det. M. Niketić, 1283 BEO, sub Viola sp.); Prijepolje: Jabuka, UTM CP70, $1100 \mathrm{~m}$, krečnjak (leg. Niketić, M., Tomović, G., Stevanović, V., Vukojičić, S. 29-Apr-2006, det. M. Niketić, BEO); 1100 m, krečnjak, kamenjari (leg. Stevanović, V., Niketić, M., Vukojičić, S., Tomović, G. 29-Apr-2006, 20863 BEOU-KEGB); S Serbia Medveđa: Tupalski vis, UTM EN53, silikat (leg. Niketić, M., 08-Oct2010, det. M. Niketić, BEO); Radan: Sokolov vis, Veliki krš, UTM EN35, silikat (leg. Niketić, M., Tomović, G. 01-May-2008, det. M. Niketić, BEO); vrh, UTM EN35, silikat (leg. Niketić, M., Tomović, G. 01-May-2008, det. M. Niketić, BEO); SE Serbia Besna kobila: Kriva Feja, UTM EN90 (leg. Niketić, M., 03-Aug-2006, det. M. Niketić, BEO); Kosovo Podujevo: brdo, UTM EN15 (leg. Košanin, N., 04-Apr-1913, 40755 BEOU); Šar-planina: Kodža Balkan, Ostrovica-Golem Bor, UTM DM87, DM97, munikova šuma (leg. Janković, M. 12-Jul-1961, 40848 BEOU). 
Viola jordanii Hanry (Fig. 6)

Banat Deliblatska peščara: Grebenac, UTM EQ17 (leg. Soška, Th., 24-Jun-1944, det. Th. Soška, 8253 BEO, sub $V$. elatior, rev. M. Niketić, 25-Sep-2013); Šumadija Beograd: Avala, UTM DQ64, DQ65 (leg. Momčilović, R., 17-May-1984, 42965 BEO, sub $V$. persicifolia, rev. M. Niketić, 25-Sep-2013); (leg. Pančić, J., det. J. Pančić, 14601 BEOU (Herb. Panc.), sub V. elatior, rev. M. Niketić, 21-Sep-2013); (leg. Soška, Th. May-1932, 45136 BEOU, sub $V$. elatior, rev. G. Tomović, 07-Sep-2013); (leg. Soška, Th. 20-Jun-1929, 45121 BEOU, sub $V$. montana, rev. M. Niketić, 07-Sep-2013); Košutnjak, UTM DQ55, 16-Oct-2008, det. M. Niketić, BEO); (leg. Petrović, S. Apr-1878, 40711 BEOU, sub $V$. elatior, rev. M. Niketić, 07-Sep2013); Kragujevac: Resnički potok, UTM DP98 (leg. Rudski, I. 15-May-1938, 8250 BEO, sub V. elatior, rev. M. Niketić, 25-Sep-2013); Šumarice, UTM DP87, 14-May-1939, 8251 BEO, rev. M. Niketić, 25-Sep-2013); (leg. Rudski, I. 24-Aug-1939, 8249 BEO, sub V. elatior, rev. M. Niketić, 25-Sep-2013); Pomoravlje Jagodina, po branicima (leg. Pančić, J., 01-May-1847, det. J. Pančić, 14602 BEOU (Herb. Panc.), sub V. elatior, rev. M. Niketić, 21-Sep-2013); Đurđevo brdo, UTM EP26

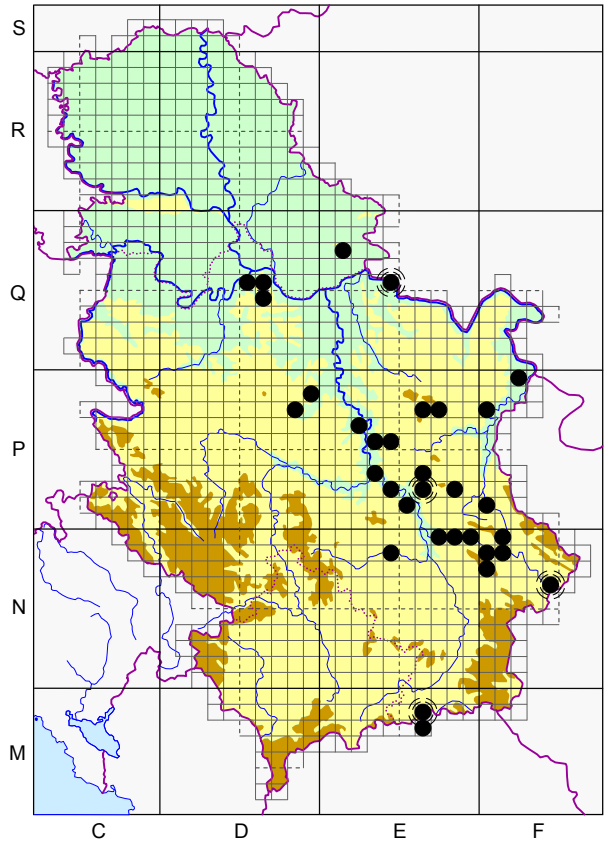

Fig. 6. - Distribution of the species

$V$. jordanii Hanry in Serbia.

(leg. Pančić, J. 11-Jun-1847, det. J. Pančić, 3229 BEOU (Herb. Panc.), sub V. elatior, rev. M. Niketić, 21Sep-2013); Paraćin: Baba planina, UTM EP45, 400-650 m, krečnjak (leg. Niketić, M., Tomović, G. 24Apr-2005, 19550 BEOU-KEGB); (leg. Niketić, M., Tomović, G. 24-Apr-2005, det. M. Niketić, BEO); Glavica, UTM EP35, 150-250 m, silikat (leg. Niketić, M., Tomović, G. 24-Apr-2005, 19454 BEOUKEGB); (leg. Niketić, M., Tomović, G. 24-Apr-2005, det. M. Niketić, BEO); Veliko Gradište, UTM EQ45, Exp. NW, krečnjak, Querco-Carpinetum (leg. Nikolić, V. 15-Apr-1959, det. M. Janković, 43121 BEO, sub V. sylvestris, rev. M. Niketić, 25-Sep-2013); NE Serbia Malinik: Lazareva reka kanjon, UTM EP67, EP77, krečnjak (leg. Lakušić, D., Tomović, G., 18-May-2005, 19728 BEOU-KEGB); Negotin: Bukovče, UTM FP29 (leg. Soška, Th. 22-Apr-1930, det. M. Niketić, 45124 BEOU, sub Viola sp.); Zaječar: Gradskovo, Čokonjarsko brdo, UTM FP07 (leg. Niketić, M., Tomović, G. 01-Jun-2005, det. M. Niketić, BEO); C Serbia Ćićevac: Stalaćka klisura, Đunis, UTM EP42, silikat (leg. Niketić, M., 26-May-1996, det. M. Niketić, BEO); Stalać-Stevanac, UTM EP33, silikat (leg. Niketić, M. 30-Apr-1996, det. M. Niketić, BEO); Prokuplje: Guba, UTM EN48, stepa (leg. Niketić, M., Tomović, G. 01-May-2002, det. M. Niketić, 15434 BEOU-KEGB, sub Viola sp.); Gubetin, UTM EN48, silikat (leg. Niketić, M., Tomović, G. 30-Apr-2008, det. M. Niketić, BEO); stepa (leg. Niketić, M., Tomović, G. 30-Apr2008, 26858 BEOU-KEGB); preko puta groblja, UTM EN48, 320 m, silikat, šikare (leg. Niketić, M., Tomović, G. 08-May-2004, det. M. Niketić, BEO); E Serbia Aleksinac, UTM EP62 (leg. Pecić, 1929, 45139 BEOU, sub V. elatior, rev. G. Tomović, 07-Sep-2013); Žitkovac, Nozrina-Smrdan, UTM EP51, neobrađeni vinogradi (leg. Bogdanović, M. 29-Apr-1973, det. M. Janković, 42822 BEO, sub V. canina, rev. M. Niketić, 25-Sep-2013); Bela Palanka: Čiflik, Ljubatovica, UTM FN18, Exp. S, šljunak (leg. Niketić, M. 18-Apr-2009, det. M. Niketić, BEO); Dol - Donja Glama, UTM FN19, stepa (leg. Niketić, M., Tomović, G. 01-May-2003, 16534 BEOU-KEGB); Dol selo, UTM FN08, stepa (leg. Niketić, M., Tomović, G. 01-May-2003, det. M. Niketić, BEO); Knjaževac: Beli Potok, UTM EP63, krečnjak, stene (leg. Niketić, M., Tomović, G. 06-Apr-2008, 26981 BEOU-KEGB); UTM EP82 (leg. Niketić, M., Tomović, G. 06-Apr-2008, det. M. Niketić, BEO); Straževica, UTM EP63 (leg. Grebenščikov, O. 15May-1935, det. O. Grebenščikov, 8254 BEO, sub V. elatior, rev. M. Niketić, 25-Sep-2013); Niš: 
Delijski vis, UTM EN79, 350 m, kompozitna podloga, šikare (leg. Niketić, M. 22-Nov-2006, det. M. Niketić, BEO); Jelašnička klisura, UTM EN89, stene (leg. Nikolić, V., Diklić, N. 24-Apr-1965, det. N. Diklić, 42830 BEO, sub $V$. canina, rev. M. Niketić, 25-Sep-2013); Kunovica, Ploča, prema Kunovičkom vrhu, UTM EN89, 500 m, krečnjak, hrastove šume i proplanci (leg. Niketić, M., Tomović, G. 08-May-2004, det. M. Niketić, BEO); Niška banja, Koritnik, UTM EN89 (leg. Lindtner, V. 09-Jun1952, det. V. Lindtner, 8246 BEO, sub V. elatior, rev. M. Niketić, 25-Sep-2013); okolina (leg. Petrović, S. 1879, 45094 BEOU, sub V. elatior, rev. M. Niketić, 07-Sep-2013); Sićevačka klisura, manastir Sveta Petka, UTM EN99 (leg. Soška, Th. Oct-1924, det. M. Niketić, 45147 BEOU, sub Viola sp.); manastir Sveta Petka, Ostrovica, UTM EN99, mešovita hrastova šuma i utrine (leg. Nikolić, V., Diklić, N. 25-Apr-1965, det. N. Diklić, 42826 BEO, sub V. canina, rev. M. Niketić, 25-Sep-2013); manastir Sveta Petka-Radov do, UTM EN99, krečnjak, šuma (leg. Lindtner, V. 02-May-1954, det. N. Diklić, 42832, 42829 BEO, sub V. canina, rev. M. Niketić, 25-Sep-2013); Suvi do - Gabrovac, Bršljan, UTM EN79, 400 m, silikat, šikare (leg. Niketić, M. 29-Oct-2013, det. M. Niketić, BEO); Suva planina: Smrdan, UTM FN07, FN08, vinograd, 40712 BEOU, rev. M. Niketić, 07-Sep-2013); Tresibaba: najviši vrh, UTM FP01, krečnjak (leg. Niketić, M., Tomović, G. 02-May-2005, 19587 BEOU-KEGB); S Serbia Preševo, UTM EM68, krečnjak, kamenjari u pojasu grabića (leg. Nikolić, V., Diklić, N., 16May-1975, det. M. Janković, 42823 BEO, sub V. canina, rev. M. Niketić, 25-Sep-2013); Rujan planina: Orljak, UTM EM67, 742 m, krečnjak, kamenjari (leg. Niketić, M., Tomović, G. 03-May-2009, 29557 BEOU-KEGB, sub V. montana, rev. M. Niketić, 21-Sep-2013); Veliki Orljak, UTM EM67, krečnjak (leg. Niketić, M., Tomović, G. 03-May-2009, det. M. Niketić, BEO); SE Serbia Dimitrovgrad, UTM FN46 (leg. Simonović, S., Jun-1923, 45146 BEOU, sub V. elatior, rev. G. Tomović, 07Sep-2013).

Viola mirabilis L. (Fig. 7)

NE Serbia Đerdapska klisura: Štrbac, UTM FQ03, FQ04 (leg. Pančić, J., 01-May1872, det. J. Pančić, 3257 BEOU (Herb. Panc.)); Kučevo: Kučajna, UTM EQ52 (leg. Pančić, J. 01-Apr-1869, det. J. Pančić, 3256 BEOU (Herb. Panc.)); W Serbia Mokra gora (leg. Pančić, J., 01-Jan-1866, det. J.Pančić, 3255 BEOU (Herb. Panc.)); Krsmanski potok, UTM CP84 (leg. Soška, Th. Jun-1914, det. W. Becker, 45141 BEOU, sub $V$. odorata, rev. M. Niketić, 07-Sep2013); Ograđenica, UTM CP74, krečnjak (leg. Jovanović, S., Niketić, M., Tomović, G. 04-Jul1998, 12626 BEOU-KEGB); Ovčarsko-Kablarska klisura, UTM DP36 (leg. Soška, Th. Jun1912, det. M. Niketić, 45125 BEOU, sub Viola sp.); Tara planina: Beli Rzav - klisura, Kršanje, UTM CP74, Exp. S, 500 m, krečnjak, šikare (leg. Niketić, M. 09-Oct-1997, det. M. Niketić, BEO); Derventa reka - kanjon, UTM CP66, krečnjak (leg. Niketić, M. 13-May1989, det. M. Niketić, BEO); (leg. Niketić, M. 14-Jul-1989, det. M. Niketić, BEO); (leg. Stevanović, V., Jovanović, S., Pavić, S. 29-May-1994, 1965-94 BEOU-KEGB); Gradac reka - kanjon, UTM CP67, $350 \mathrm{~m}$, krečnjak, šikare (leg. Niketić, M., Jovanović, S., Tomović, G. 03-Jul1998, det. M. Niketić, BEO); C Serbia Kopaonik: Treska, UTM DN88 (leg. Rudski, I., 14-Jul-1938, 8182 BEO); Kraljevo: Bogutovac-Gornja Lopatnica, Lopatnica reka - klisura, UTM DP52, serpentinit (leg. Stevanović, V., Niketić, M., Vukojičić, S., Tomović, G. 30-Apr-2004, 18588 BEOU-KEGB); E Serbia Niš: Jelašnička klisura, Jelašnica, Krš, UTM EN89, Exp. N (leg. Petrović, S., 1875,

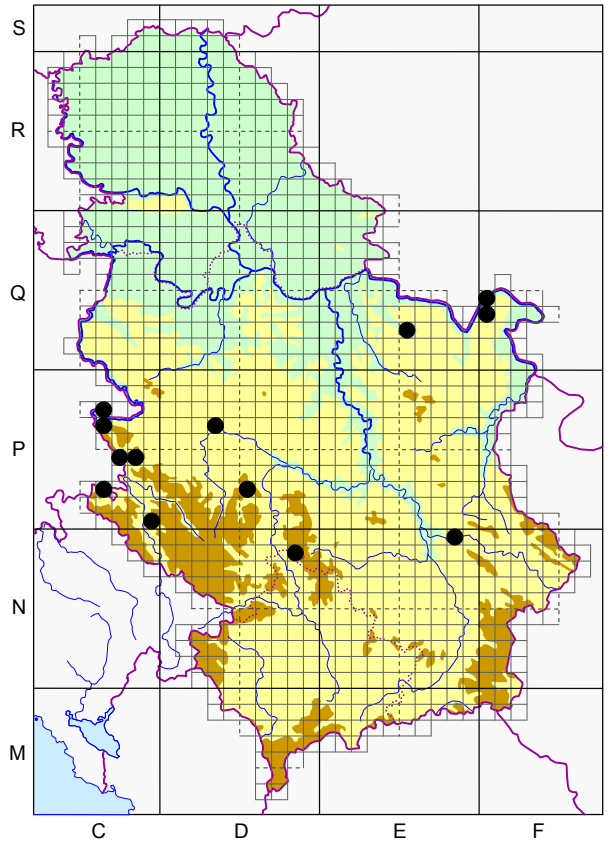

Fig. 7. - Distribution of the species $V$. mirabilis L. in Serbia. 


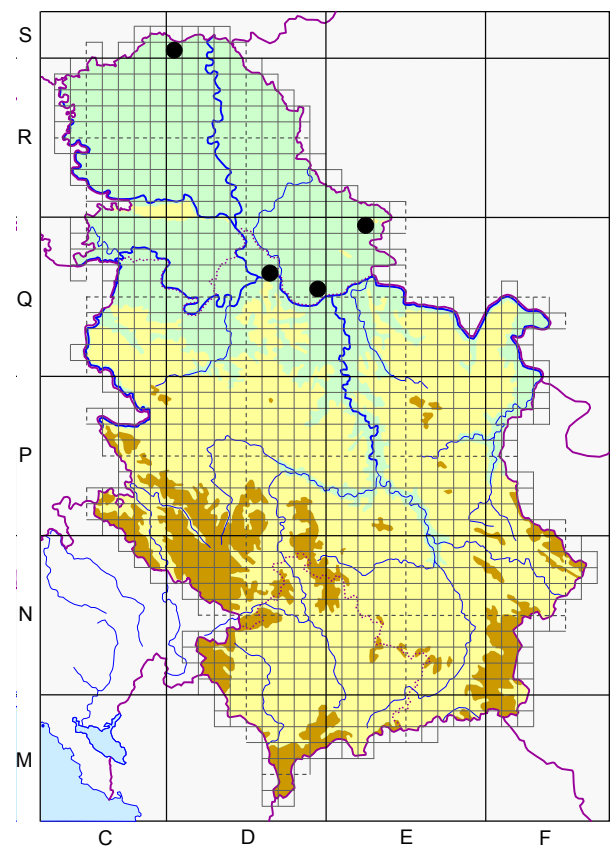

Fig. 8. - Distribution of the species V. obliqua Hill. in Serbia.

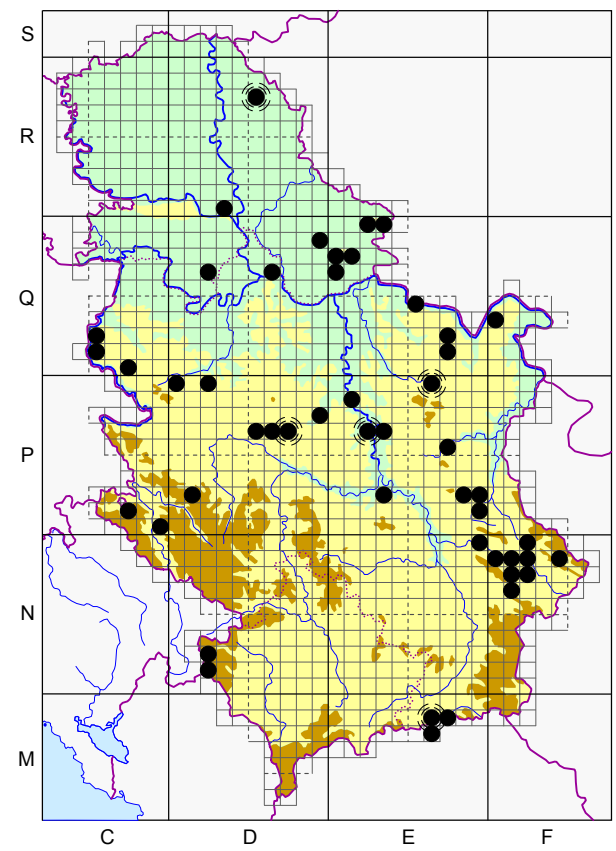

Fig. 9. - Distribution of the species $V$. odorata L. in Serbia.
41031 BEOU); SW Serbia Priboj: Sjeverin, Sutjeska reka - klisura, UTM CP62, 500-700 m, krečnjak (leg. Niketić, M., Tomović, G., 13-Aug-2008, det. M. Niketić, BEO); Prijepolje: Mileševka reka -kanjon, UTM CP90, krečnjak, stene (leg. Stevanović, V., Niketić, M., Vukojičić, S., Tomović, G. 29-Apr-2006, 20834 BEOU-KEGB).

Viola obliqua Hill. (Fig. 8)

Bačka Ludaško jezero: Ludaški šor, UTM DS00, 110.3 m (leg. Lakušić, D., Lakušić, B., 29-Apr-2008, 26566 BEOU-KEGB); Subotica: Hajdukovo, Rasadnik Gold, UTM DS00, 107.6 m (leg. Lakušić, D., Lakušić, B. 29-Apr-2008, 26565 BEOU-KEGB); Banat Kovin, UTM DQ95, poljana pored groblja (leg. Jovanović, E., 30-Apr-2002, 40952 BEOU, sub V. hirta, rev. M. Niketić, 07-Sep-2013); Vršac, UTM EQ29, 03-Apr-1927, det. V. Tereščenko, 1293 BEO, rev. M. Niketić, 25-Sep-2013); Šumadija Beograd: Zvezdara, UTM DQ66 (leg. Lakušić, D., 06-Jul-2006, 20937 BEOU-KEGB).

\section{Viola odorata L. (Fig. 9)}

Banat Deliblatska peščara (leg. Grebenščikov, O., 04-Apr-1948, det. M. Niketić, 1288 BEO, sub Viola sp.); (leg. Lakušić, D. 16-Apr1988, 345 BEOU-KEGB); Čardak, UTM EQ17 (leg. Stevanović, V., Jovanović, S., Lakušić, D., Vukojicicí, S. 26-Apr-1997, 4932 BEOU-KEGB, sub V. suavis, rev. M. Niketić, 21-Sep-2013); Deliblato, UTM EQ06 (leg. Niketić, M., Stevanović, V., Tomović, G., Vukojičić, S. 12-Apr-2009, det. M. Niketić, BEO); Devojački bunar, UTM DQ98, bukova šuma (leg. Stevanović, $V$. 19-Mar-1974, det. V. Stevanović, 40880 BEOU, sub V. suavis, rev. M. Niketić, 07-Sep-2013); hrastova šuma (leg. Stevanović, V. 24-Mar-1974, det. V. Stevanović, 40879 BEOU, sub V. suavis, rev. M. Niketić, 07-Sep-2013); QuercoTilietum tomentosae (leg. Stevanović, V., Jovanović, S., Lakušić, D. 26-Mar-1990, 10-90 BEOUKEGB, sub $V$. suavis, rev. M. Niketić, 07-Sep2013); (leg. Nikolić, V., Diklić, N. 03-Apr-1971, det. N. Diklić, 42951, 42952 BEO); Devojački bunar - Flamunda, UTM EQ07 (leg. Diklić, N. 03-Apr-1970, det. N. Diklić, 42942, 42943, 42944, 42945 BEO); (leg. Nikolić, V., Diklić, N., Bogdanović, M. 27-Mar-1970, det. N. Diklić, 42946, 42947, 42948, 42949 BEO); Kikinda, UTM DR57 (leg. Izmajlov 19-Apr-1922, det. 
Izmajlov, $8140 \mathrm{BEO}$ ); Vršac, 02-Apr-2000, 40741 BEOU); Vršačke planine: Odmaralište, UTM EQ29, šume kitnjaka i lipe (leg. Nikolić, V., Diklić, N. 06-May-1970, det. M. Janković, 42751 BEO, sub $V$. $a l b a$, rev. M. Niketić, 25-Sep-2013); planinarski dom, Lisičji vrh, UTM EQ29 (leg. Nikolić, V., Diklić, N. 17-Jul-1969, det. N. Diklić, 43093 BEO, sub V. suavis, rev. M. Niketić, 25-Sep-2013); Velika čoka, UTM EQ39, mešovite šume kitnjaka i lipe (leg. Nikolić, V., Diklić, N. 07-May-1970, det. M. Janković, 42750 BEO, sub V. alba, rev. M. Niketić, 25-Sep-2013); Srem Fruška gora: Slankamen, Koševac, UTM DR30, Exp. N, 220 m, les, mezofilne livade (leg. Niketić, M., Tomović, G., 13-Apr-2014, det. M. Niketić, BEO); Pećinci: Karlovčić, revir, UTM DQ26 (leg. Stanojević, S. 07-Apr-1996, det. S. Stanojević, 45078 BEOU); (leg. Stanojević, S. 25-Apr-1996, det. S. Stanojević, 40893 BEOU); NW Serbia Gučevo: Crni vrh, UTM CQ52, Exp. S, 400-600 m, krečnjak, Ostryetum (leg. Niketić, M., Tomović, G., 26-Apr-2008, det. M. Niketić, BEO); Mali Zvornik: Orlovine-Grabovac, UTM CQ51, krečnjak (leg. Niketić, M., Tomović, G. 14-Nov-2010, det. M. Niketić, BEO); Mionica: Ribnica klisura, manastir Pastić, UTM DP29, krečnjak (leg. Niketić, M., Tomović, G. 29-Mar-2008, det. M. Niketić, BEO); Podrinje: Sokolska planina, Carine, Erića kamen, UTM CQ70, krečnjak (leg. Niketić, M. 12-May-2010, det. M. Niketić, BEO); Soko grad, UTM CQ70, krečnjak + dolomitizirani krečnjak (leg. Niketić, M. 29-Apr-2010, det. M. Niketić, BEO); Šumadija Beograd (leg. Milošević, S., 1990, 40954 BEOU, rev. M. Niketić, 07-Sep-2013); Višnjica, UTM DQ66 (leg. Lindtner, V. 25-Mar1934, 1546 BEO); (leg. Lindtner, V. 25-Mar-1934, 1532 BEOU-KEGB, sub V. alba, rev. M. Niketić, 07Sep-2013); Gornji Milanovac: Vujan, Mali Vujan, UTM DP56, šume, Quercetalia pubescentis (leg. Lakušić, D., Vukojičić, S., Jakovljević, K., Kuzmanović, N. 01-Apr-2009, det. M. Niketić, 28824 BEOU-KEGB, sub Viola sp.); Knić, UTM DP76 (leg. Rudski, I. 14-Apr-1940, 1301 BEO; 1303 BEO, sub V. alba, rev. M. Niketić, 25-Sep-2013); Gornji Vujetinci, Oštrica brdo, UTM DP66, Exp. N, 650-802 m, silikat (andezit) (leg. Niketić, M., Tomović, G. 16-Apr-2004, det. M. Niketić, BEO); Kragujevac: Petrovac, UTM DP97 (leg. Rudski, I. 17-Apr-1940, det. I. Rudski, 8146 BEO, sub V. sepincola, rev. M. Niketić, 25Sep-2013); Pomoravlje Ćuprija, UTM EP26 (leg. Pančić, J., 06-Apr-1847, det. J. Pančić, 3264 BEOU (Herb. Panc.)); Velika Morava: Bagrdan klisura, Brzan, UTM EP18, silikat (leg. Niketić, M. 09-Apr-2010, det. M. Niketić, BEO); NE Serbia Đerdapska klisura: Golubac, Golubačko grad, UTM EQ54, šuma grabića (leg. Nikolić, V., Diklić, N., 15-May-1967, det. N. Diklić, 42756, 42757 BEO, sub V. alba, rev. M. Niketić, 25-Sep-2013); Štrbac, Veliki Štrbac, UTM FQ03, 600 m, mešovita šuma (leg. Diklić, N. 10-Oct-1963, det. M. Janković, 42752 BEO, sub V. alba, rev. M. Niketić, 25-Sep-2013); Majdanpek: Debeli lug, Veliki Pek - klisura, UTM EQ71, krečnjak (leg. Niketić, M. 08-Apr-2010, det. M. Niketić, BEO); Veliki Pek - klisura, kod sela, UTM EQ71, 150 m, krečnjak, stene (leg. Niketić, M., Tomović, G. 09-Jul-2009, det. M. Niketić, BEO); Majdanpečka Domena, Felješana reka, UTM EQ71 (leg. Vasiljević, K. 10-Jul-1948, BEO, sub V. sylvestris, rev. M. Niketić, 25-Sep-2013); Rajkova pećina, UTM EQ72 (leg. Stevanović, V. 25-Apr-1997, 4959 BEOU-KEGB); Starica, UTM EQ72, šume i kamenjari (leg. Pavlović, S. 16-Apr-1876, 40701 BEOU, sub V. suavis, rev. M. Niketić, 07-Sep2013); greben, UTM EQ72, krečnjak (leg. Niketić, M. 04-Jul-2010, det. M. Niketić, BEO); Žagubica, UTM EP69 (leg. Pančić, J. 01-Jan-1869, det. J. Pančić, 3266 BEOU (Herb. Panc.)); W Serbia Arilje: Veliki Rzav - klisura, UTM DP12 (leg. Lakušić, D., Jovanović, S., 29-Jul-1990, 2252-90 BEOU-KEGB, sub V. alba, rev. M. Niketić, 21-Sep-2013); C Serbia Trstenik: Pasjak, UTM EP32, $300 \mathrm{~m}$ (leg. Spasojević, J., 14-Mar-1992, det. D. Lakušić, 45080 BEOU, sub V. alba, rev. M. Niketić, 07-Sep-2013); E Serbia Bela Palanka: Čiflik, Ljubatovica, UTM FN18, Exp. S, šljunak (leg. Niketić, M., 18-Apr2009, det. M. Niketić, BEO); Divljan selo, klisura, UTM FN08, krečnjak (leg. Stevanović, V., Zlatković, B., Tomović, G., Vukojičić, S., Jušković, M. 07-Apr-2004, 22440 BEOU-KEGB, sub V. alba, rev. M. Niketić, 07-Sep-2013); Crni Timok: Mali Izvor, UTM EP75, krečnjak, stena (leg. Jovanović, S. 15Apr-1995, 1532 BEOU-KEGB, sub V. sylvestris, rev. M. Niketić, 25-Sep-2013); Knjaževac: Beli Potok, UTM EP82 (leg. Niketić, M., Tomović, G. 06-Apr-2008, det. M. Niketić, BEO); Svrljiški Timok - klisura, UTM EP91, EP92 (leg. Niketić, M., Tomović, G. 06-Apr-2008, det. M. Niketić, BEO); Niš: okolina (leg. Petrović, S. 1879, det. M. Niketić, 45221 BEOU, sub Viola sp.); (leg. Petrović, S., 41053 BEOU, sub V. collina, rev. M. Niketić, 07-Sep-2013); Sićevačka klisura, Oblik, UTM EN99, Carpinetum orientalis (leg. Niketić, M. 03-Mar-2007, det. M. Niketić, BEO); (leg. Niketić, M. 07-Apr-2007, det. M. Niketić, BEO); (leg. Niketić, M. 09-Jul-2005, det. M. Niketić, BEO); Paraćin: Grza reka klisura, kod planinarskog doma, UTM EP36, krečnjak, livada (leg. Pejanović, V. 25-Apr-2001, 40743 BEOU); Pirot: Babušnica, UTM FN16, FN17, Exp. S, Querco-Carpinetum (leg. Nikolić, V. 25-Mar1979, det. M. Janković, 42940 BEO); Kostur, UTM FN27, Exp. S, krečnjak, Querco-Carpinetum (leg. Nikolić, V. 31-Mar-1979, det. M. Janković, 42938 BEO); Sarlak, UTM FN27, FN28, Exp. S, krečnjak, 


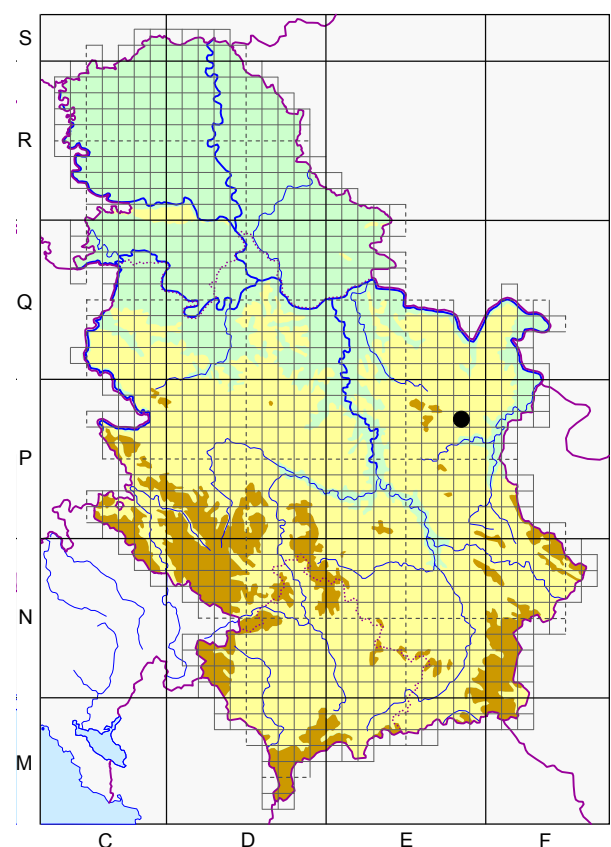

Fig. 10. - Distribution of the species $V$. pumila Chaix in Serbia.

Querco-Carpinetum (leg. Nikolić, V. 24-Mar1979, det. M. Janković, 42936, 42937, 42941 BEO); Sinjac, UTM FN18, krečnjak (leg. Niketić, M. 04-Apr-2009, det. M. Niketić, BEO); Temska, UTM FN29, Exp. NW, krečnjak, Querco-Carpinetum (leg. Nikolić, V. 26-Mar1979, det. M. Janković, 42939 BEO, rev. M. Niketić, 25-Sep-2013); Stara planina: Visočica klisura, UTM FN48 (leg. Cvetković, $D$. 20-Jul-1991, 2678-91 BEOU-KEGB); Svrljiške planine: Krupac, istočno od sela, UTM DP09, Exp. N, 400 m, krečnjak, kamenjari i stene (leg. Niketić, M. 01-Mar-2014, det. M. Niketić, BEO); SW Serbia Priboj: Ožalj, UTM CP71 (leg. Lindtner, V., 14-Apr-1949, det. M. Niketić, 1272 BEO, sub Viola sp.); Prijepolje: Mileševka reka - kanjon, manastir Mileševa, iznad, UTM CP90, hrastova šuma (leg. Pavlović, Z. 14-Apr-1949, det. M. Niketić, 1292 BEO, sub Viola sp.); S Serbia Preševo, UTM EM68, mešovita hrastova šuma i kamenjar (leg. Nikolić, V., Diklić, N., 22-Apr1965, det. N. Diklić, 42950 BEO); Rujan planina: Veliki Orljak, UTM EM67, krečnjak (leg. Niketić, M., Tomović, G. 04-Apr-2010, det. M. Niketić, BEO); SE Serbia Pčinja

(klisura): manastir Prohor Pčinjski, UTM EM78, krečnjak (leg. Stevanović, V., Zlatković, B., Tomović, G., Vukojičić, S., 05-Apr-2004, det. M. Niketić, 22486 BEOU-KEGB, sub Viola sp.); Metohija Prokletije: Prilepska planina, Raški do-Savine vode-Amšor-Žljeb-Kovraga, UTM DN21, DN22 (leg. Janković, M., 28May-07-Jun-1960, det. M. Niketić, 40874 BEOU, sub Viola sp.).

Viola pumila Chaix (Fig. 10)

NE Serbia Bor: Brestovačka banja, UTM EP87 (leg. Pančić, J., 01-Apr-1869, det. J. Pančić, 3276 BEOU (Herb. Panc.), sub $V$. pumila (pratensis), rev. M. Niketić, 07-Sep2013).

Viola reichenbachiana Jord. ex Boreau (Fig. 11)

Banat Deliblatska peščara: Deliblato, UTM EQ06, pesak, peččara (leg. Soška, Th., 26Sep-1922, 45126 BEOU, sub V. canina, rev. M. Niketić,07-Sep-2013);Zagajačko brdo, UTM EQ17, les (leg. Stevanović, V., Vukojičić, S., Jakovljević, K. 12-Apr-2009, det. M. Niketić, 29413 BEOU-KEGB, sub Viola sp.); Vršačke planine: Lisičji vrh, UTM EQ29, šuma bukve,

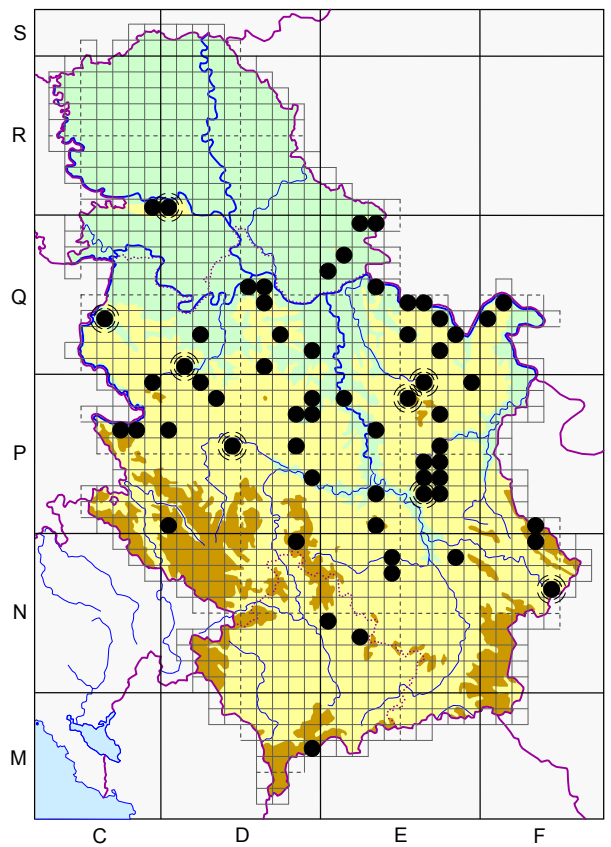

Fig. 11. - Distribution of the species $V$. reichenbachiana Jord. ex Boreau in Serbia. 
graba i lipe (leg. Nikolić, V., Diklić, N. 06-May-1970, det. N. Diklić, 43147 BEO, sub V. sylvestris, rev. M. Niketić, 25-Sep-2013); Velika čoka, UTM EQ39, 600-641 m, mešovita šuma kitnjaka i lipe (leg. Nikolić, V., Diklić, N. 07-May-1970, det. N. Diklić, 43146 BEO, sub V. sylvestris, rev. M. Niketić, 25-Sep2013); Srem Beočin: Veliki čot, UTM CR90, hrastova šuma (leg. Janković, M., 28-Jun-1954, det. M. Niketić, 40878 BEOU, sub Viola sp., rev. M. Niketić, 07-Sep-2013); Fruška gora, UTM DR00 (leg. Černjavski, P. 13-May-1938, det. P. Černjavski, 1549 BEO, sub V. sylvestris, rev. M. Niketić, 25-Sep2013); NW Serbia Jablanik, UTM CP99, bukova šuma (leg. Diklić, N., 23-Apr-1955, det. M. Janković, 43113 BEO, sub V. sylvestris, rev. M. Niketić, 25-Sep-2013); (leg. Nikolić, V., Diklić, N. 25-May1954, det. M. Janković, 43109 BEO, sub V. sylvestris, rev. M. Niketić, 25-Sep-2013); Exp. E, bukova šuma (leg. Nikolić, V., Diklić, N. 25-May-1954, det. M. Janković, 42976 BEO, sub V. rupestris, rev. M. Niketić, 25-Sep-2013); Štabulja, UTM CP99, bukova šuma (leg. Nikolić, V., Diklić, N. 26-May-1954, det. M. Janković, 43111 BEO, sub V. sylvestris, rev. M. Niketić, 25-Sep-2013); Jablanik-Medvednik: Japadak, UTM CP99, Exp. SE, bukova šuma (leg. Nikolić, V., Diklić, N. 26-May-1954, det. M. Janković, 43115 BEO, sub V. sylvestris, rev. M. Niketić, 25-Sep-2013); Loznica, UTM CQ63 (leg. Novaković, A. Mar-1872, 40703 BEOU, sub V. suavis, rev. M. Niketić, 18-Sep-2013); Mionica: Ribnica - klisura, manastir Pastić, UTM DP29, krečnjak (leg. Niketić, M., Tomović, G. 29-Mar-2008, det. M. Niketić, BEO); Valjevo, UTM DQ10, Apr-1875, det. M. Niketić, 40955 BEOU); Jablanica reka - klisura, manastir Pustinja, UTM CP99, 450 m, krečnjak, stene (leg. Niketić, M. 24-Apr-1992, det. M. Niketić, BEO); Šumadija Aranđelovac: Venčac, severne padine, UTM DQ60, Exp. N, $550 \mathrm{~m}$, silikat i mermer (leg. Niketić, M., Tomović, G., 16-Apr-2005, det. M. Niketić, BEO); Beograd: Avala, UTM DQ64, DQ65, borova kultura (leg. Nikolić, V. 11-Apr-1952, det. V. Nikolić, 1557 BEO, sub V. sylvestris, rev. M. Niketić, 25-Sep-2013); hrastovo-grabova šuma (leg. Borisavljević, Lj. 18-Apr-1952, 40769, 40770, 40771 BEOU, sub V. sylvatica, rev. M. Niketić, 07-Sep-2013); Exp. NW, šuma kitnjaka i graba (leg. Diklić, N. 27-Apr-1970, det. M. Niketić, 1566 BEO, sub Viola sp.); smrčeva šuma (leg. Nikolić, V. 14-Apr1953, det. V. Nikolić, 1558 BEO, sub V. sylvestris, rev. M. Niketić, 25-Sep-2013); (03-Jul-1952, 40768 BEOU, sub V. sylvatica, rev. M. Niketić, 07-Sep-2013); (leg. Borisavljević, Lj. 14-May-1952, 40775 BEOU, sub $V$. sylvatica, rev. M. Niketić, 07-Sep-2013); (leg. Mišić, V. 15-May-1952, det. M. Niketić, 40764, 40765, 40766 BEOU, sub Viola sp.); Banjica, UTM DQ55, Apr-1878, 40704 BEOU, rev. M. Niketić, 07-Sep-2013); Kaluđerica, UTM DQ65, Exp. NE, šibljak (leg. Blečić, V. 07-Apr-1938, 1550 BEO, sub V. sylvestris, rev. M. Niketić, 25-Sep-2013); Kievački potok, UTM DQ55, šibljak (leg. Rajevski, L. 06-Apr-1938, 1547 BEO, sub V. sylvestris, rev. M. Niketić, 25-Sep-2013); Klenje, UTM DQ64 (leg. Nikolić, V., Lindtner, V. 19-Apr-1952, det. M. Niketić, 1563 BEO, sub Viola sp.); železnička stanica, UTM DQ64 (leg. Nikolić, V., Lindtner, V. 19-Apr-1952, det. M. Niketić, 1554, 1564 BEO, sub Viola sp.); Košutnjak, UTM DQ55, lipovo-grabova šuma (leg. Grebenščikov, O. 01-Apr-1945, 1515 BEO, sub V. canina, rev. M. Niketić, 25-Sep-2013); (leg. Đelii, B. 20-Oct-1936, 1518 BEO, sub V. canina, rev. M. Niketić, 25-Sep-2013); (leg. Janković, M., Mešiček 1952, det. M. Niketić, 40861 BEOU, sub Viola sp.); (leg. Jurišić, J. 15-Apr-1924, det. J. Jurišić, 1552 BEO, sub V. sylvestris, rev. M. Niketić, 25-Sep2013); (leg. Lakušić, D. 10-May-1986, 949-86 BEOU-KEGB, sub V. sylvestris, rev. M. Niketić, 07-Sep2013); (leg. Rajevski, L. 01-May-1937, 1551 BEO, sub V. sylvestris, rev. M. Niketić, 25-Sep-2013); Knić: Balosave, UTM DP85, šibljak (leg. Delić, Z. 14-Apr-1949, det. V. Peričić, 8226 BEO, sub V. sylvestris, rev. M. Niketić, 25-Sep-2013); Kragujevac (leg. Rudski, I. 25-Mar-1939, 8218 BEO, sub V. sylvestris, rev. M. Niketić, 25-Sep-2013); (leg. Rudski, I. 28-Mar-1937, 8225 BEO, sub V. sylvestris, rev. M. Niketić, 25-Sep-2013); Badnjevac, Šupljaja, UTM DP98 (leg. Rudski, I. 30-Jul-1939, 8228 BEO, sub V. sylvestris, rev. M. Niketić, 25-Sep-2013); Košutnjak, UTM DP97 (leg. Rudski, I. 22-May-1937, 1278 BEO, sub V. sylvestris, rev. M. Niketić, 25-Sep-2013); Mečkovac, UTM DP97 (leg. Rudski, I. 02-Apr, 8234 BEO, sub V. sylvestris, rev. M. Niketić, 25-Sep-2013); (leg. Rudski, I. 02-Apr-1936, det. Z. Pavlović, 8222 BEO, sub V. sylvestris, rev. M. Niketić, 25-Sep-2013); Petrovac, UTM DP97, šuma (leg. Rudski, I., 1316 BEO, sub V. odorata, rev. M. Niketić, 25-Sep-2013); (leg. Rudski, I. 17-Apr-1940, det. I. Rudski, 8146 BEO, sub V. sepincola, rev. M. Niketić, 25-Sep-2013); Šumarice, UTM DP87 (leg. Rudski, I. 10-Mar-1937, det. M. Niketić, 1315 BEO, sub Viola sp.); Vašarište, UTM DP97, $190 \mathrm{~m}$ (leg. Marinković, D. 20-Apr-1997, det. V. Stevanović, 40740 BEOU, sub V. sylvestris, rev. M. Niketić, 07Sep-2013); Mladenovac: Mala Vrbica, UTM DQ72, hrastova šuma (leg. Vukojičić, S., Tomović, G. 19Mar-2002, 14897 BEOU-KEGB, sub V. sylvestris, rev. M. Niketić, 07-Sep-2013); Suvobor: Vijuljak, UTM DP38, 920 m, serpentiniti, kamenjari (leg. Niketić, M., Jovanović, M. 06-May-2004, det. M. Niketić, BEO); Ub, 1926, det. M. Niketić, 45157 BEOU); Crvena jabuka, UTM DQ22, Querco-Carpinetum 
(leg. Stevanović, V., Jovanović, S., Lakušić, D., Vukojičić, S. 12-Mar-1997, 4891 BEOU-KEGB, sub V. odorata, rev. M. Niketić, 07-Sep-2013); Pomoravlje Smederevska Palanka, šuma (leg. Tomić, A., 30-Jul-1991, det. S. Jovanović, 41060 BEOU, sub V. sylvestris, rev. M. Niketić, 07-Sep-2013); Glibovac, UTM DQ91, šuma (leg. Tomić, A. 10-May-1992, det. S. Jovanović, 41073 BEOU, sub $V$. sylvestris, rev. M. Niketić, 07-Sep-2013); (leg. Tomić, A. 24-Mar-1992, det. A. Tomić, 41061 BEOU, sub V. sylvestris, rev. M. Niketić, 07-Sep-2013); (leg. Lindtner, V. 07-Apr-1950, det. V. Lindtner, 8220 BEO, sub V. sylvestris, rev. M. Niketić, 25-Sep-2013); Velika Morava: Bagrdan klisura, Bagrdan, UTM EP18, silikat, Quercetum frainetto-cerris (leg. Niketić, M., Tomović, G. 17-Mar-2007, det. M. Niketić, BEO); Brzan, UTM EP18, silikat (leg. Niketić, M. 09-Apr-2010, det. M. Niketić, BEO); Veliko Gradište: Zatonjska peščara, UTM EQ35, pesak, peščara (leg. Stevanović, V., Lakušić, D., Vukojičić, S., Tomović, G. 11-Apr-2005, 19346 BEOU-KEGB); NE Serbia Beljanica, UTM EP58, bukova šuma (leg. Diklić, N., 24-Sep-1969, det. N. Diklić, 43148 BEO, sub V. sylvestris, rev. M. Niketić, 25-Sep-2013); Bor: Stol, UTM EP99, Exp. N, bukova šuma (leg. Nikolić, V., Diklić, N. 28-Jul-1962, det. V. Nikolić, 43156 BEO, sub V. sylvestris, rev. M. Niketić, 25-Sep-2013); Exp. SE, bukova šuma (leg. Nikolić, V., Diklić, N. 29-Jun-1961, det. N. Diklić, 43155 BEO, sub V. sylvestris, rev. M. Niketić, 25-Sep-2013); Đerdapska klisura: Brnjica, UTM EQ64, šuma (leg. Nikolić, V., Diklić, N. 20-Apr-1968, det. V. Nikolić, 43162 BEO, sub V. sylvestris, rev. M. Niketić, 25-Sep-2013); Donji Milanovac, UTM EQ82, hrastova šuma (leg. Nikolić, V., Diklić, N. 23-Apr-1968, det. V. Nikolić, 43169 BEO, sub V. sylvestris, rev. M. Niketić, 25-Sep-2013); Kadin potok, UTM EQ82, 70-100 m, bukova šuma sa orahom (leg. Nikolić, V., Diklić, N., Rakin, M. 14-Sep-1965, det. N. Diklić, 43158 BEO, sub V. sylvestris, rev. M. Niketić, 25-Sep2013); Varoški potok, UTM EQ82, Exp. NW, 90 m, bukova šuma (leg. Nikolić, V., Diklić, N., Rakin, M. 11Sep-1965, det. N. Diklić, 43157 BEO, sub V. sylvestris, rev. M. Niketić, 25-Sep-2013); Golubac, UTM EQ54, šuma (leg. Nikolić, V., Diklić, N. 28-Apr-1968, det. V. Nikolić, 43164 BEO, sub V. sylvestris, rev. M. Niketić, 25-Sep-2013); Pesača potok, UTM EQ73, šuma (leg. Nikolić, V., Diklić, N. 21-Apr-1968, det. V. Nikolić, 43163 BEO, sub V. sylvestris, rev. M. Niketić, 25-Sep-2013); (leg. Nikolić, V., Diklić, N. 28Apr-1968, det. V. Nikolić, 43165 BEO, sub V. sylvestris, rev. M. Niketić, 25-Sep-2013); (leg. Nikolić, V., Diklić, N. 28-Jun-1967, det. V. Nikolić, 43161 BEO, sub V. sylvestris, rev. M. Niketić, 25-Sep-2013); Štrbac, Veliki Štrbac, Pecka bara, UTM FQ03, mešovite šume (leg. Nikolić, V., Diklić, N. 23-Apr-1976, det. M. Janković, 43127, 43126 BEO, sub V. sylvestris, rev. M. Niketić, 25-Sep-2013); Tekija, Kovilovski potok, UTM FQ14 (leg. Nikolić, V., Diklić, N. 24-Apr-1968, det. V. Nikolić, 43167 BEO, sub V. sylvestris, rev. M. Niketić, 25-Sep-2013); (leg. Nikolić, V., Diklić, N. 26-Jun-1967, det. V. Nikolić, 43166 BEO, sub V. sylvestris, rev. M. Niketić, 25-Sep-2013); Kučevo: Kučajna, UTM EQ52 (leg. Pančić, J. 01-Apr-1869, det. J. Pančić, 3284 BEOU (Herb. Panc.), sub V. sylvatica, rev. M. Niketić, 07-Sep-2013); Majdanpek: Debeli lug, UTMEQ71, bukovašuma(leg. Černjavski,P.26-Jul-1946, det.P. Černjavski, 8230 BEO, sub V. sylvestris, rev. M. Niketić, 25-Sep-2013); (31-Jul-1947, 8231 BEO, rev. M. Niketić, 25-Sep2013); Majdanpečka Domena, UTM EQ71, 300-450 m, bukova šuma, 09-Jul-1948, det. N. Diklić, 43172 BEO, rev. M. Niketić, 25-Sep-2013); proređena bukova šuma, 27-Jul-1948, det. N. Diklić, 43137 BEO, rev. M. Niketić, 25-Sep-2013); Potkapine, UTM EQ71 (leg. Černjavski, P. 14-Jul-1947, det. M. Niketić, 1269 BEO, sub Viola sp.); Todorova reka, kraj, UTM EQ71, 09-Jul-1947, 8232 BEO, rev. M. Niketić, 25-Sep-2013); Malinik, UTM EP77, 900 m, bukovo-jelova šuma (leg. Diklić, N., Miladinović, Lj. 24-Jun-1964, det. N. Diklić, 43153 BEO, sub V. sylvestris, rev. M. Niketić, 25-Sep-2013); (Jul-1986, BEOU-KEGB, rev. M. Niketić, 07-Sep-2013); Žagubica, UTM EP69, šuma (leg. Blečić, V. Jun-1943, 41089 BEOU, sub $V$. sylvestris, rev. M. Niketić, 07-Sep-2013); W Serbia Čačak, UTM DP45 (leg. S. $P$., det. M. Niketić, 40717 BEOU, sub Viola sp.); Tara planina, 40795 BEOU, rev. M. Niketić, 07-Sep2013); Kaluđerske bare, UTM CP86, 1200 m, šuma bukve i jele (leg. Nikolić, V., Bogdanović, M. 17May-1974, det. M. Janković, 43114 BEO, sub V. sylvestris, rev. M. Niketić, 25-Sep-2013); Mitrovac, UTM CP76, 1200 m, livade (leg. Diklić, N., Bogdanović, M. 17-May-1974, det. M. Janković, 43107 BEO, sub V. sylvestris, rev. M. Niketić, 25-Sep-2013); Užice: Jelova gora, UTM DP06, $1100 \mathrm{~m}$ (leg. Vukojičić, S. 03-May-1996, det. M. Niketić, 45081 BEOU, sub Viola sp.); C Serbia Jastrebac: Leva Reka, UTM EP30 (leg. Sigunov, A., 03-Aug-1949, det. M. Niketić, 1289 BEO, sub Viola sp.); Velika Đulica, UTM EP30 (leg. Sigunov, A. 31-Jul-1949, det. M. Niketić, 1284 BEO, sub Viola sp.); Veliki Jastrebac, UTM EP30, izdanačka bukova šuma (leg. Diklić, N. 12-Jun-1959, det. N. Diklić, 43139 BEO, sub V. sylvestris, rev. M. Niketić, 25-Sep-2013); Prokop-Stracimir, UTM EP30, šuma breze i bukve (leg. Ostojić, D. 20-Aug-2006, 24828 BEOU-KEGB, sub V. sylvestris, rev. M. Niketić, 07-Sep2013); Kopaonik: Novoselske bačije, Golo Brdo, UTM DN89, 1300 m, granit, kamenjari, sipari i 
stene (leg. Stevanović, V., Jovanović, S., Lakušić, D. 21-Apr-1990, det. D. Lakušić, 105-90 BEOU-KEGB); Trstenik: Pasjak, UTM EP32, 300 m (leg. Vukojičić, S. 20-Apr-1992, det. D. Lakušić, J. Spasojević, 45079 BEOU, sub V. riviniana, rev. M. Niketić, 07-Sep-2013); Vidojevica, UTM EN47, EN48, bukova šuma (leg. Nikolić, V. 15-May-1959, det. M. Janković, 43128 BEO, sub V. sylvestris, rev. M. Niketić, 25Sep-2013); Vrnjačka banja: Ljubostinja, UTM DP93 (leg. Pančić, J. 06-Apr-1847, det. J. Pančić, 3289 BEOU (Herb. Panc.), sub V. sylvestris (canina), rev. M. Niketić, 07-Sep-2013); E Serbia Aleksinac, UTM EP62 (leg. Pecić, 1929, det. M. Niketić, 45138 BEOU, sub Viola sp.); Soko Banja, UTM EP63, EP73, šibljak Corylus avelana (leg. Diklić, N. 20-Jun-1957, det. N. Diklić, 43170 BEO, sub V. sylvestris, rev. M. Niketić, 25-Sep-2013); Boljevac: Jablanica, Radovanska reka, UTM EP75, 285 m, Carpino orientalis-Quercetum mixtum (leg. Jovanović, S. 15-Apr-1995, det. M. Niketić, 1509 BEOU-KEGB, sub Viola sp.); Devica: Visoki skokar, UTM EP72, bukova šuma (leg. Diklić, N. 24May-1957, det. N. Diklić, 43154 BEO, sub V. sylvestris, rev. M. Niketić, 25-Sep-2013); Paraćin: Grza reka - klisura, Gornje jezero, UTM EP36, krečnjak (leg. Pejanović, V. 26-Apr-2001, 40744 BEOU, sub V. sylvestris, rev. M. Niketić, 07-Sep-2013); Pirot: Visočica klisura, UTM FN39 (leg. Cvetković, D. 20-Jul-1991, 3018 BEOU-KEGB, sub V. sylvestris, rev. M. Niketić, 07-Sep-2013); Rtanj, UTM EP64, EP74 (leg. Soška, Th. Jun-1929, det. M. Niketić, 45123, 45142, 45148 BEOU, sub Viola sp.); (leg. Soška, Th. 20-Apr-1930, det. M. Niketić, 45064 BEOU, sub Viola sp.); Stara planina: Dojčino vrelo, UTM FP30, Exp. NW, šume i šibljaci (leg. Nikolić, V. 05-May-1979, det. M. Janković, 43120 BEO, sub V. sylvestris, rev. M. Niketić, 25-Sep-2013); Suva planina: Mosor, UTM EN88, bukova šuma (leg. Stevanović, V., Lakušić, D. 07-Apr-1990, 25-91 BEOU-KEGB, sub V. sylvestris, rev. M. Niketić, 07-Sep2013); SW Serbia Zlatar: Babića brdo-Mokra poljana, Nova Varoš, kod, UTM DP00, smrčeve šume (leg. Nikolić, V., Diklić, N., 21-Jun-1970, det. N. Diklić, 43145 BEO, sub V. sylvestris, rev. M. Niketić, 25-Sep-2013); SE Serbia Dimitrovgrad, UTM FN46 (leg. Simonović, S., Jun-1928, 45122 BEOU, sub V. sylvatica, rev. M. Niketić, 07-Sep-2013); Kosovo Priština: Kačikol, UTM EN23, bukova šuma (leg. Nikolić, V., 01-Jun-1961, det. V. Nikolić, 40763 BEOU, sub V. sylvestris, rev. M. Niketić, 07-Sep-2013); Šar-planina: Prevalac, UTM DM96, bukove sastojine (leg. Janković, M. 09Jun-1965, det. M. Niketić, 40875 BEOU, sub Viola sp.); Vučitrn: Skočna, UTM EN04 (leg. Košanin, N. Apr-1913, 45211 BEOU, sub V. sylvestris, rev. M. Niketić, 07-Sep-2013).

Viola riviniana Rchb. (Fig. 12) Banat Deliblatska peščara: Vrela, UTM EQ17, pesak, peščara (leg. Stevanović, V., Jovanović, S., Lakušić, D., Vukojičić, S., Tomović, G., 30Sep-1997, det. M. Niketić, 7579 BEOU-KEGB, sub Viola sp.); Vršačke planine: Vršački breg, Lisičji vrh, UTM EQ29, šume (leg. Nikolić, V., Diklić, N. 26-Jun-1968, det. N. Diklić, 43150 BEO, sub V. sylvestris, rev. M. Niketić, 25-Sep-2013); Srem (leg. Pančić, J., det. J. Pančić, 3287 BEOU (Herb. Panc.), sub $V$. sylvestris, rev. M. Niketić, 21-Sep-2013); NW Serbia Cer planina, UTM CQ74, CQ83, bukova šuma, (Apr-1988, 41088 BEOU, sub $V$. sylvatica, rev. M. Niketić, 19-Sep-2013); (11May-1984, sub Viola sp., det. M. Niketić, 41068 BEOU); Gučevo: Crni vrh, UTM CQ52 (leg. Vukićević, M. 28-Jul-1910, det. Ž. J. Jurišić, 8241 BEO, sub $V$. canina, rev. M. Niketić, 25-Sep-2013); Gavrina reka, UTM CQ52, laporac (leg. Niketić, M. 07-Apr-2010, det. M. Niketić, BEO); Jablanik, UTM CP99, bukova šuma (leg. Nikolić, V. 10-May-1955, det. M. Janković, 43116 BEO, sub V. sylvestris, rev.

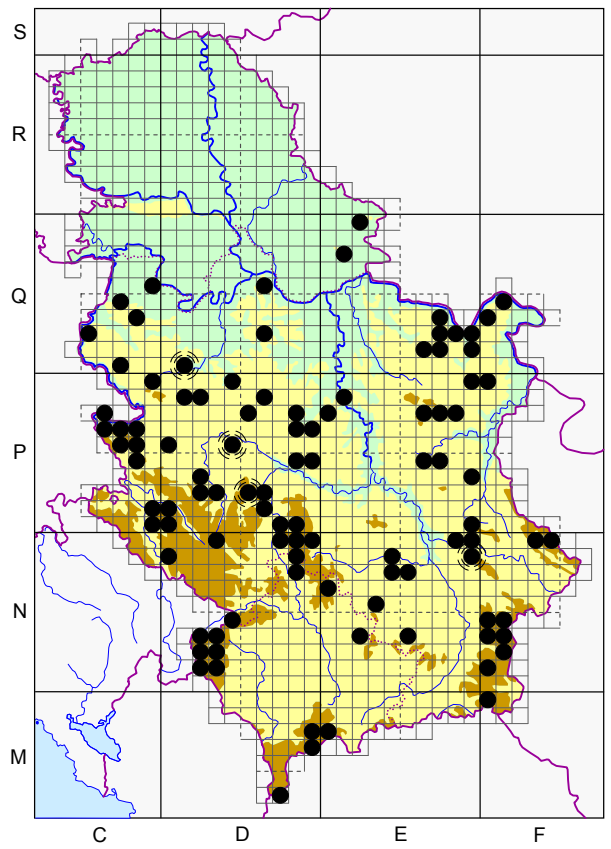

Fig. 12. - Distribution of the species $V$. riviniana Rchb. in Serbia. 
M. Niketić, 25-Sep-2013); (leg. Nikolić, V. 12-May-1955, det. M. Janković, 43108 BEO, sub V. sylvestris, rev. M. Niketić, 25-Sep-2013); (leg. Nikolić, V., Diklić, N. 27-May-1954, det. M. Janković, 43118, 43112 BEO, sub V. sylvestris, rev. M. Niketić, 25-Sep-2013); (leg. Nikolić, V., Diklić, N. 27-Jun-1954, det. M. Janković, 43117 BEO, sub V. sylvestris, rev. M. Niketić, 25-Sep-2013); livade (leg. Nikolić, V., Diklić, N. 24-May-1954, det. M. Janković, 42977 BEO, sub V. rupestris, rev. M. Niketić, 25-Sep-2013); pašnjak (leg. Nikolić, V., Diklić, N. 24-May-1954, det. M. Janković, 42978 BEO, sub V. rupestris, rev. M. Niketić, 25-Sep-2013); planinarski dom, UTM CP99, Exp. NE, papratište (leg. Diklić, N. 25-Apr-1955, 42974 BEO); retka cerova šuma (leg. Diklić, N. 23-Apr-1955, 43138 BEO, sub V. sylvestris, rev. M. Niketić, 25Sep-2013); Maljen, UTM DP18, DP28 (leg. Pančić, J. 01-Jun-1861, det. J. Pančić, 3292 BEOU (Herb. Panc.), sub $V$. sylvestris var. riviniana, rev. M. Niketić, 21-Sep-2013); Medvednik, UTM CP99, bukova šuma (leg. Nikolić, V. 11-May-1955, det. M. Janković, 43110 BEO, sub V. sylvestris, rev. M. Niketić, 25-Sep-2013); vrh, UTM CP99, bukova šuma (leg. Nikolić, V. 11-May-1955, det. N. Diklić, 43101 BEO); Podrinje: Sokolska planina, Soko grad, UTM CQ70, dolomit (leg. Niketić, M. 29Apr-2010, det. M. Niketić, BEO); Šabac: Jelenovačka šuma, UTM CQ95, šuma (leg. Lindtner, V. 28-Mar-1950, det. M. Niketić, 1285 BEO, sub Viola sp.); Valjevo, UTM DQ10, 40839 BEOU, rev. M. Niketić, 07-Sep-2013); Šumadija Beograd: Avala, Beli Potok, UTM DQ65, hrastovi šibljak (leg. Nikolić, V., 26-Apr-1954, det. M. Niketić, 1555 BEO, sub Viola sp.); šibljak Quercetum (leg. Diklić, N. 26Apr-1954, det. N. Diklić, 1560 BEO, sub V. sylvestris, rev. M. Niketić, 25-Sep-2013); Gledićke planine: Medveđa kosa, UTM DP94 (leg. Rudski, I. 26-Apr-1940, 8206 BEO); Gornji Milanovac: Brđanska klisura, UTM DP57, serpentinit, Stipetum novakii i šuma medunca (leg. Stevanović, V., Jovanović, S., Pavić, S. 30-May-1994, det. M. Niketić, 2041-94 BEOU-KEGB, sub Viola sp.); Rudnik, UTM DP68, 640 m (leg. Lindtner, V. 16-May-1950, det. M. Niketić, 1254 BEO, sub Viola sp.); Kragujevac: Beloševac, UTM DP96 (leg. Rudski, I. 02-May-1939, 8217 BEO, sub V. sylvestris, rev. M. Niketić, 25-Sep-2013); (leg. Rudski, I. 30-May-1937, det. M. Niketić, BEO, sub Viola sp.); Crni vrh, UTM EP07, 2310 m (leg. Rudski, I. 12-Jul-1930, 41080 BEOU); Čukojevački gaj, Ćirkovićev zabran, UTM DP84, peščani useci (leg. Rudski, I. 16-Jun-1940, 8208 BEO); Dragobraća, UTM DP86 (leg. Rudski, I. 23-Apr-1939, 8204 BEO); Popova šuma, šuma (leg. Rudski, I. 29-Apr-1935, 8193 BEO); Poskurička šuma, UTM DP87, šuma (leg. Rudski, I. 12-Apr-1939, 8223 BEO, sub V. sylvestris, rev. M. Niketić, 25-Sep-2013); Šumarice, UTM DP87 (leg. Rudski, I. 14-May-1939, 8219 BEO, sub V. sylvestris, rev. M. Niketić, 25-Sep-2013); Vitanovac, UTM DP84 (leg. Rudski, I. 07-Apr-1938, 8207 BEO); Ljig: Štavica, UTM DP49, Querco-Carpinetum (leg. Stevanović, V., Lakušić, D., Ostojić, D. 28Apr-1992, 10-92 BEOU-KEGB, sub V. sylvestris, rev. M. Niketić, 19-Sep-2013); Mladenovac: Kosmaj, UTM DQ62 (leg. Bogdanović, M. 24-Oct-1971, det. M. Janković, 42973 BEO); Pomoravlje Velika Morava: Bagrdan klisura, manastir Janković-manastir Tomić, UTM EP18, silikat (leg. Niketić, M., Tomović, G., 17-Mar-2007, det. M. Niketić, BEO); NE Serbia Bor: Brestovačka banja, UTM EP87 (leg. Pančić, J., 01-Jul-1876, det. M. Niketić, 3190 BEOU (Herb. Panc.), sub Viola sp.); Stol, UTM EP99, bukova šuma (leg. Nikolić, V. 25-May-1955, 43132 BEO, sub V. sylvestris, rev. M. Niketić, 25-Sep-2013); Exp. SE, bukova šuma (leg. Nikolić, V. 24-May-1955, 43130 BEO, sub $V$. sylvestris, rev. M. Niketić, 25-Sep-2013); Deli Jovan: Goli vrh, UTM FP09, kamenjar (leg. Nikolić, V. 26-Jul-1956, 43135 BEO, sub V. sylvestris, rev. M. Niketić, 25-Sep-2013); Đerdapska klisura: Avramica, UTM EQ92, livade (leg. Niketić, M., Tomović, G. 14-Jun-2009, det. M. Niketić, BEO); Donji Milanovac, UTM EQ82, Exp. NW, 80 m, bukova šuma (leg. Nikolić, V., Diklić, N., Rakin, M. 11-Sep-1965, det. N. Diklić, 43159 BEO, sub V. sylvestris, rev. M. Niketić, 25-Sep-2013); Veliki greben, UTM EQ91, EQ92, bukova šuma (leg. Nikolić, v. 21-Jun-1956, 43136 BEO, sub V. sylvestris, rev. M. Niketić, 25-Sep-2013); po šumama-po dumacima (leg. Pavlović, S. 20-Apr-1875, 40702 BEOU, sub V. canina, rev. M. Niketić, 07-Sep-2013); (leg. Pančić, J. 01-Apr-1875, det. J. Pančić, 3267 BEOU (Herb. Panc.), sub $V$. odorata, rev. M. Niketić, 21-Sep-2013); Miroč, Konjska glava, UTM FQ03, 500 m, krečnjak, SyringoCarpinion orientalis (leg. Stevanović, V., Lakušić, D., Niketić, M. 10-May-1996, 43-96 BEOU-KEGB, sub V. sylvestris, rev. M. Niketić, 19-Sep-2013); Pesača potok, UTM EQ73, mešovite i bukove šume (leg. Nikolić, V., Diklić, N. 22-Apr-1976, det. M. Janković, 43123, 43124 BEO, sub V. sylvestris, rev. M. Niketić, 25-Sep-2013); Štrbac, Veliki Štrbac, UTM FQ03, hrastovo-bukova šuma (leg. Nikolić, V., Diklić, N. 19May-1967, det. V. Nikolić, 43160 BEO, sub V. sylvestris, rev. M. Niketić, 25-Sep-2013); (leg. Stevanović, V., Vukojičić, S., Tomović, G., Lazarević, P. 20-Apr-2002, det. M. Niketić, 14833 BEOU-KEGB, sub Viola sp.); Veliki Štrbac, lugarnica-vrh, UTM FQ03, šuma (leg. Nikolić, V., Diklić, N. 25-Apr-1968, det. V. Nikolić, 43168 BEO, sub V. sylvestris, rev. M. Niketić, 25-Sep-2013); Tekija, UTM FQ14 (leg. Pančić, 
J. 01-Sep-1877, det. J. Pančić, 3285 BEOU (Herb. Panc.), sub V. sylvatica, rev. M. Niketić, 21-Sep2013); Majdanpek: Majdanpečka Domena, UTM EQ71, čiste bukove sastojine (leg. Diklić, N. 16Jul-1948, 43171 BEO, sub V. sylvestris, rev. M. Niketić, 25-Sep-2013); Kraku Mustafa, UTM EQ61, EQ71, mešovita šuma (leg. Gojković, U. 22-Jul-1947, det. M. Niketić, 1241 BEO, sub Viola sp.); Potkapine, UTM EQ71, hrastova šuma (leg. Černjavski, P. 14-Jul-1947, det. N. Diklić, 43173 BEO, sub V. sylvestris, rev. M. Niketić, 25-Sep-2013); šuma, 28-Jul-1947, 1242 BEO, rev. M. Niketić, 25-Sep2013); Starica, UTM EQ72, šuma (leg. Pavlović, S. 12-Apr-1875, 40838 BEOU, sub V. sylvestris, rev. M. Niketić, 19-Sep-2013); Malinik: Lazareva reka - kanjon, Gaura Lazaru, UTM EP67, EP77 (leg. Pančić, J. 01-Jan-1867, det. M. Niketić, 3181 BEOU (Herb. Panc.), sub Viola sp.); Miroč: Semolj površ, UTM FQ03, 03-May-1984, 1767-89 BEOU-KEGB, rev. M. Niketić, 19-Sep-2013); W Serbia Čačak, UTM DP45 (leg. Pančić, J., 01-Apr-1851, det. J. Pančić, 3290 BEOU (Herb. Panc.), sub $V$. sylvestris, rev. M. Niketić, 21-Sep-2013); (leg. Pančić, J. 01-May-1852, det. J. Pančić, 3277 BEOU (Herb. Panc.)); Čemerno, UTM DP52 (leg. Pančić, J. 01-Aug-1860, det. M. Niketić, 3178, 3179 BEOU (Herb. Panc.), sub Viola sp.); Ivanjica: Crvena gora, UTM DP32, 01-May-1996, det. M. Niketić, 45085 BEOU); Panjica reka - klisura, UTM DP23, 400 m, krečnjak, Orno-Ostryetum (leg. Stanić S., Benić, N. 01-Aug-1990, det. M. Niketić, 2989 BEOU-KEGB, sub Viola sp.); Mučanj: Katići, UTM DP22, stene, kamenjari, šuma (leg. Lakušić, D. 17-Oct-1990, 2836 BEOU-KEGB, sub V. sylvestris, rev. M. Niketić, 19-Sep-2013); Nova Varoš: Kokin brod, UTM DP01, krečnjak, smrčeva šuma (leg. Niketić, M., Tomović, G. 02-Jul-2006, det. M. Niketić, BEO); Rasadi, UTM DP01, livada (leg. llić, S. 25-Jun1930, 41075 BEOU); Tara planina: Bilo - rezervat, UTM CP66 (leg. Stevanović, V., Jovanović, S., Vukojičić, S. 12-Jul-2000, det. M. Niketić, 14421 BEOU-KEGB, sub Viola sp.); Bilješke stene, UTM CP67, krečnjak (leg. Stevanović, V., Jovanović, S., Lakušić, D. 31-May-1997, 5150 BEOU-KEGB, sub V. sylvestris, rev. M. Niketić, 19-Sep-2013); Kaluđerske bare-Kremna, UTM CP85, CP86, 900-1100 $\mathrm{m}$, serpentinit, borova šuma (leg. Diklić, N., Bogdanović, M. 09-May-1974, det. M. Janković, 42972 BEO); Kremna, UTM CP85 (leg. Jovanović, S., Jovanović, B. 07-Aug-2004, det. M. Niketić, 22117 BEOUKEGB, sub Viola sp.); Mitrovac, Crveni potok, UTM CP76, $1060 \mathrm{~m}$ (leg. Stevanović, V., Jovanović, S. 23-May-2001, det. M. Niketić, 13769 BEOU-KEGB, sub Viola sp.); (1094 m, mešovite močvarne šume jove i omorike, leg. Kuzmanović, N., Đurović, S., Janković, I. 02-Jun-2012, det. M. Niketić, 35651 BEOUKEGB, sub Viola sp.); Rača reka - kanjon, UTM CP86, jelovo-bukova šuma (leg. Diklić, N., Rakin, M. 17-Jul-1963, det. N. Diklić, 43141 BEO, sub V. sylvestris, rev. M. Niketić, 25-Sep-2013); (leg. Diklić, N. 18-Jul-1963, 43140 BEO, sub V. sylvestris, rev. M. Niketić, 25-Sep-2013); Zborište, Zmajevski potok, UTM CP75 (leg. Ostojić, D. 28-Aug-1999, det. M. Niketić, 9575 BEOU-KEGB, sub Viola sp., rev. M. Niketić, 21-Sep-2013); Užice: Buar, Gornja Pora, UTM DP05, 660 m, 01-May-1996, det. S. Vukojičić, 45083 BEOU, rev. M. Niketić, 07-Sep-2013); Užice-Kremna, UTM CP85, 42971 BEO); Zlatibor, krečnjak, 16-May-1931, 45056 BEOU, rev. M. Niketić, 18-Sep-2013); Crni Rzav - klisura, Crni vrh, UTM CP84, bukova šuma (leg. Nikolić, V. 01-Aug-1955, 43131 BEO, sub V. sylvestris, rev. M. Niketić, 25-Sep-2013); (leg. Nikolić, V. 27-May-1955, 43134, 43133 BEO, sub V. sylvestris, rev. M. Niketić, 25-Sep-2013); Zvezda: Crni vrh, Crvena stena, UTM CP66, šume i stene u šumi sa Pančićevom omorikom (leg. Stevanović, V., Jovanović, S., Vukojičić, S. 11-Jul-2000, det. M. Niketić, 14672 BEOU-KEGB, sub Viola sp.); Podstolac, Štula karaula, UTM CP66 (leg. Pančić, J. 01-May-1880, det. M. Niketić, 3207 BEOU (Herb. Panc.), sub Viola sp.); C Serbia Ibarska dolina: Raška, Rudine, UTM DN79, Exp. E, 300 m, serpentiniti, šikare (leg. Niketić, M., Stevanović, V., Tomović, G., Vukojičić, S., 02-May-2004, det. M. Niketić, BEO); Ušće, UTM DP61, serpentinit, kamenjari (leg. Stevanović, V., Vukojičić, S., Tomović, G. 14-Jun-2003, det. M. Niketić, 16803 BEOU-KEGB, sub Viola sp.); Kopaonik, Exp. NW, 1570 m, gusta smrčeva šuma, 1952, det. M. Niketić, 40785 BEOU); bukovosmrčeva šuma (leg. Rudski, I. 31-May-1938, det. I. Rudski, 8205 BEO); subalpska smrča (leg. Popović, M. 26-Jun-1954, det. M. Niketić, 40762, 40761 BEOU, sub Viola sp.); (leg. Mišić, V., Popović, M. 1952, det. M. Niketić, 40780, 40779 BEOU, sub Viola sp.); (leg. Pančić, J. 01-May-1875, det. J. Pančić, 3224 BEOU (Herb. Panc.), sub V. canina, rev. M. Niketić, 07-Sep-2013); (leg. Pančić, J. 01-May-1875, det. J. Pančić, 14998 BEOU (Herb. Panc.), sub V. sylvestris var. riviniana, rev. M. Niketić, 21-Sep-2013); (leg. Rudski, I. 10-Jul-1938, 8224 BEO, sub V. sylvestris, rev. M. Niketić, 25-Sep-2013); Bećirovac, Duboka reka, UTM DN89, Exp. N, NE, 1680 m, bukovo-jelovo-smrčeva šuma, 30-Jul-1952, det. M. Niketić, 40772 BEOU); Exp. S, 1690 m, smrčeva šuma, 30-Jul-1952, det. M. Niketić, 40773 BEOU); Exp. NW, 1585 m, serpentinit, bukova šuma sa vacciniumom, det. M. Niketić, 40774 BEOU); Duboka reka donja, UTM DN89, Exp. E, 1080 m, 31-Jul-1952, det. M. Niketić, 40782 BEOU); Bele stene, Srebrnac, UTM 
DN89, 1650 m, subalpijske bukove šume, 08-Aug-1952, det. M. Niketić, 40781 BEOU); (1700 m, krečnjak, rudine, leg. Stevanović, V., Jovanović, S., Lakušić, D. 20-Apr-1990, det. D. Lakušić, 93-90 BEOUKEGB, sub V. reichenbachiana, rev. M. Niketić, 20-Sep-2013); Drenjska klisura, UTM DP80, Exp. SW, 750 m, granodiorit, termofilne hrastove šume (leg. Lakušić, D., Niketić, M. 18-Apr-1986, det. D. Lakušić, 40798 BEOU, sub V. sylvestris, rev. M. Niketić, 19-Sep-2013); Gobelja, Oštri krš, UTM DN89, 1700m m, krečnjak (leg. Stevanović, V., Niketić, M. 04-Jun-1985, det. D. Lakušić, 40707 BEOU, sub V. sylvestris, rev. M. Niketić, 18-Sep-2013); Jošanička banja, Zablaća, UTM DP70, serpentinit, borova šuma (leg. Borisavljević, Lj. 06-May-1954, det. M. Niketić, 40846 BEOU, sub Viola sp.); ZablaćeJarebica, UTM DP70, kamenjar (leg. Borisavljević, Lj. 06-May-1954, det. L. Rajevski, 40845 BEOU); Quercetum montanum (leg. Rajevski, L., Borisavljević, Lj. 06-May-1954, det. M. Niketić, 40844 BEOU, sub Viola sp.); Kaljevac, Šanac, UTM DN79, hrastova šuma (leg. Rajevski, L. 23-Apr-1952, det. L. Rajevski, 40851 BEOU, sub V. sylvestris, rev. M. Niketić, 07-Sep-2013); šibljak (leg. Rajevski, L. 23-Apr1953, det. L. Rajevski, 40859 BEOU, sub V. sylvestris, rev. M. Niketić, 18-Sep-2013); (leg. Rajevski, L. 24-Apr-1953, det. L. Rajevski, 40860 BEOU, sub V. sylvestris, rev. M. Niketić, 18-Sep-2013); Karaman, UTM DN89, 1750m m, granit, progala smrčeve šume (leg. Stevanović, V., Niketić, M. 04-Jun1985, det. D. Lakušić, 40708 BEOU, sub V. sylvestris, rev. M. Niketić, 18-Sep-2013); (leg. Stevanović, V., Niketić, M. Jun-1985, det. D. Lakušić, 40705 BEOU, sub V. sylvestris, rev. M. Niketić, 18-Sep-2013); Samokovska reka - klisura, UTM DN89, Exp. N, 1510 m, tresava (leg. Mišić, V., Popović, M. 09-Jun1952, det. M. Niketić, 40778 BEOU, sub Viola sp.); Exp. SW, W, 1555 m, smrčeve šume, 23-Jul-1952, det. M. Niketić, 40789 BEOU); (leg. Stevanović, V., Niketić, M. Jun-1985, det. D. Lakušić, 40706 BEOU, sub V. sylvestris, rev. M. Niketić, 18-Sep-2013); Suvi jelak, UTM DN89, Exp. NW, 1670 m, gusta mlada smrčeva šuma, 16-Jul-1952, det. M. Niketić, 40788 BEOU); Suvi jelak-Babin grob, UTM DN89, 1600 m, 07-Jun-1952, det. M. Niketić, 40784 BEOU); Treska, UTM DN88 (leg. Rudski, I. 14Jul-1938, 8233 BEO, sub V. sylvestris, rev. M. Niketić, 25-Sep-2013); Vlajkovci, Brzećka reka, UTM DN99, serpentinit (leg. Niketić, M., Tomović, G. 14-May-2011, 32133 BEOU-KEGB, sub V. canina, rev. M. Niketić, 20-Sep-2013); Vučak, UTM DN89, Exp. NE, 1570 m, smrčeva šuma, 12-Aug-1952, det. M. Niketić, 40783 BEOU); Kraljevo: Čukojevac, UTM DP84 (leg. Rudski, I. 25-Apr-1940, 8203 BEO); (leg. Rudski, I. 27-Apr-1940, 8192 BEO); Dobre strane, Dobre strane-vrh, UTM DP62 (leg. Slavković, Ž. Mar-1970, det. N. Diklić, 43144 BEO, sub V. sylvestris, rev. M. Niketić, 25-Sep-2013); Pasjača, UTM EN47, EN57, bukova šuma (leg. Diklić, N. 16-Jul-1959, det. M. Janković, 42970 BEO); Vidojevica, UTM EN47, EN48, bukova šuma (leg. Nikolić, V. 15-May-1959, det. M. Janković, 43119 BEO, sub V. sylvestris, rev. M. Niketić, 25-Sep-2013); E Serbia Knjaževac: Bučje, UTM EP93 (leg. Pančić, J., 01-May-1875, det. M. Niketić, 3291 BEOU (Herb. Panc.), sub Viola sp.); Niš: okolina (leg. Petrović, S. 1879, 40718 BEOU, sub V. sylvestris, rev. M. Niketić, 18-Sep-2013); Sićevačka klisura, Kusača, UTM EN89, mešovite šume i stene (leg. Nikolić, V., Diklić, N. 26-Apr-1965, det. N. Diklić, 43151 BEO, sub V. sylvestris, rev. M. Niketić, 25-Sep-2013); manastir Sveta Petka, UTM EN99 (leg. Lindtner, V. 13-May-1937, 1276 BEO, sub V. elatior, rev. M. Niketić, 25-Sep-2013); Rtanj, UTM EP64, EP74, 1300- 1530 m, pašnjaci (leg. Diklić, N. 08-Jun-1965, det. N. Diklić, 43142 BEO, sub $V$. sylvestris, rev. M. Niketić, 25-Sep-2013); Stara planina, 41084 BEOU, rev. M. Niketić, 19-Sep-2013); Topli do, UTM FN49, šumarak (leg. Grebenščikov, O. 11-May-1948, det. O. Grebenščikov, U. Rajnović, 40852 BEOU, sub V. canina, rev. M. Niketić, 18-Sep-2013); Belan, UTM FN39, 1550 m, pašnjak (leg. Grebenščikov, O. 13-May-1948, det. O. Grebenščikov, U. Rajnović, 40853 BEOU); Suva planina, UTM EN98, 41083 BEOU, rev. M. Niketić, 19-Sep-2013); Svrljiške planine: Pleš, UTM EP90 (leg. Pančić, J. 01-Jan-1868, det. J. Pančić, 3275 BEOU (Herb. Panc.), sub V. prov. elatior, rev. M. Niketić, 21-Sep2013); SW Serbia Giljeva: Krajinovići, Čelo brdo, UTM DN08, Exp. NE, 1000 m, serpentinit (leg. Stevanović, V., Niketić, M., Vukojičić, S., Tomović, G., 28-Apr-2006, det. M. Niketić, 20725 BEOUKEGB, sub Viola sp.); Exp. NW, 1000 m, serpentinit (leg. Niketić, M., Tomović, G., Stevanović, V., Vukojičić, S. 28-Apr-2006, det. M. Niketić, BEO); Pešter: Jarut, UTM DN39 (leg. Košanin, N. Jun-1926, det. M. Niketić, 45060 BEOU, sub Viola sp.); Priboj: Dobrun, Pešterci zaseok, UTM CP91, krečnjak (leg. Niketić, M., Tomović, G. 14-Aug-2008, det. M. Niketić, BEO); Prijepolje: Mileševka reka - kanjon, UTM CP90, krečnjak (leg. Niketić, M., Tomović, G., Stevanović, V., Vukojičić, S. 23-Apr-2006, det. M. Niketić, BEO); krečnjak, stene (leg. Stevanović, V., Niketić, M., Vukojičić, S., Tomović, G. 29-Apr-2006, det. M. Niketić, 20840 BEOU-KEGB, sub Viola sp.); Zlatar: Babića brdo-Mokra poljana, Nova Varoš, kod, UTM DP00, 1200-1400 m, smrčeve šume (leg. Nikolić, V., Diklić, N. 19-Jun-1970, det. N. Diklić, 43149 BEO, sub V. sylvestris, rev. M. Niketić, 25-Sep-2013); S Serbia Medveđa: Tupalski 
vis, UTM EN53, silikat (leg. Niketić, M., 08-Oct-2010, det. M. Niketić, BEO); Radan, bukova šuma (leg. Nikolić, V. 11-May-1959, det. M. Janković, 43122 BEO, sub V. sylvestris, rev. M. Niketić, 25-Sep2013); Sokolov vis, Mali krš, UTM EN35, silikat (leg. Niketić, M., Tomović, G. 01-May-2008, det. M. Niketić, BEO); Veliki krš, UTM EN35, silikat (leg. Niketić, M., Tomović, G. 01-May-2008, det. M. Niketić, BEO); SE Serbia Bosilegrad: Valozi, UTM FN01, 1700-1800 m, silikat (leg. Niketić, M., Tomović, G., Zlatković, B., Anačkov, G., 15-Aug-2006, det. M. Niketić, BEO); Ostrozub, UTM FN04, 07Aug-1950, 40890 BEOU, rev. M. Niketić, 19-Sep-2013); (leg. Popović, M. Aug-1950, det. M. Niketić, 40871 BEOU, sub Viola sp.); (leg. Popović, V. 06-Aug-1950, det. M. Niketić, 40872, 40873 BEOU, sub Viola sp.); Pčinja - klisura: Radovnica, UTM FM09 (leg. Nikolić, V., Diklić, N. 15-Jul-1972, det. M. Janković, 43129 BEO, sub V. sylvestris, rev. M. Niketić, 25-Sep-2013); Vlasina (leg. Pančić, J. 01-Jan1880, det. M. Niketić, 3205 BEOU (Herb. Panc.), sub Viola sp.); Jerma klisura, Klisura selo ispred, UTM FN12, FN13, Exp. SE, mešovita šuma bukve, cera, kitnjaka i graba (leg. Jovanović, S., Niketić, $M$. 30-Aug-1991, 1947-91 BEOU-KEGB, sub V. reichenbachiana, rev. M. Niketić, 20-Sep-2013); Plana, UTM FN04, FN14, bukov šibljak (leg. Černjavski, P., Pavlović, Z., Mišić, V. 28-May-1947, det. M. Niketić, 40855 BEOU, sub Viola sp.); Vrtop, UTM FN13, Exp. NW (leg. Jovanović, S., Niketić, M. 29-Aug-1991, 1731-91 BEOU-KEGB, sub V. reichenbachiana, rev. M. Niketić, 20-Sep-2013); Vlasinsko jezero, UTM FN03, šume breze, bukve (leg. Nikolić, V., Diklić, N. 13-17-Jun-1967, det. N. Diklić, 43143 BEO, sub V. sylvestris, rev. M. Niketić, 25-Sep-2013); Kosovo Ibarska dolina: Raška, Leposavić, UTM DN87, serpentinit (leg. Stevanović, V., Niketić, M., Vukojičić, S., Tomović, G., 02-May-2004, 18770 BEOUKEGB, sub V. mirabilis, rev. M. Niketić, 20-Sep-2013); Kopaonik: Oštro koplje, Bare, UTM EN06 (leg. Soška, Th. 29-Jun-1914, det. Th. Soška, 45234 BEOU); Priština: Kačikol, UTM EN23, bukova šuma sa javorom (leg. Nikolić, V. 01-Jun-1961, 43102 BEO); Šar-planina: Brezovica, Borov vrh, UTM DM97, 1170 m, serpentinit, kamenjari (leg. Lazarević, M., Lazarević, P. 15-Aug-2011, 33137 BEOUKEGB, sub V. canina, rev. M. Niketić, 20-Sep-2013); Careve livade, UTM DM96, molikove šume (leg. Janković, M. 08-Jul-1961, 40869 BEOU, sub V. sylvestris, rev. M. Niketić, 19-Sep-2013); munika i beli bor (leg. Janković, M. 08-Jul-1961, 40864 BEOU, sub V. sylvestris, rev. M. Niketić, 18-Sep-2013); Gine vode, UTM DM96, molikova šuma (leg. Janković, M. 07-Jul-1961, 40790 BEOU, sub $V$. sylvestris, rev. M. Niketić, 19-Sep-2013); (leg. Janković, M. 08-Jul-1961, 40865 BEOU, sub V. sylvestris, rev. M. Niketić, 18-Sep-2013); (leg. Janković, M. 08-Jul-1961, 40866 BEOU, sub V. sylvestris, rev. M. Niketić, 19-Sep-2013); Gine vode-Prevalac-Štrpce, UTM EM07 (leg. Janković, M. 08-11-Jun-1965, det. M. Niketić, 40759 BEOU, sub Viola sp.); Kodža Balkan, Ostrovica, UTM DM97, silikat (leg. Janković, M. 15-Jul-1967, 40786 BEOU, sub V. sylvestris, rev. M. Niketić, 19-Sep-2013); silikat, krečnjak (i serpentin), munika (leg. Janković, M. 16-Aug-1967, 40887 BEOU, sub V. sylvestris, rev. M. Niketić, 19Sep-2013); Kokošinje, UTM EM07, 1200 m, krečnjak, Taxo-Fagetum (leg. Stevanović, V. 01-Sep-1997, det. M. Niketić, 7844 BEOU-KEGB, sub Viola sp.); Ošljak, Bela voda, UTM DM97 (leg. Soška, Th. 07-Jun-1920, 45219 BEOU, sub V. sylvestris, rev. M. Niketić, 07-Sep-2013); Popovo prase-Ostrovica, UTM DM97 (leg. Stevanović, V. 26-Jun-1997, det. M. Niketić, 7279 BEOU-KEGB, sub Viola sp.); Prevalac, UTM DM96, bukove sastojine (leg. Janković, M. 09-Jun-1965, det. M. Niketić, 40760 BEOU, sub Viola sp.); Metohija Mokra gora: Radovački stanovi, UTM DN44, bukova šuma (leg. Janković, M., 02-May-1960, det. M. Niketić, 40850 BEOU, sub Viola sp.); krečnjak, bukova šuma (leg. Janković, M. 02-May-1960, det. M. Niketić, 40841 BEOU, sub Viola sp.); Prokletije: Boge, UTM DN23, šuma belog bora (leg. Janković, M. 15-Jul-1958, det. M. Niketić, 40891 BEOU, sub Viola sp.); Dečanska Bistrica, Dečani, UTM DN31, Exp. E, 700 m, kestenova šuma (leg. Popović 14-Jun-1970, det. M. Niketić, 40854 BEOU, sub Viola sp.); Exp. E, 800 m, kestenove šume (leg. Janković, M. 29-May1959, 40793 BEOU, sub $V$. sylvestris, rev. M. Niketić, 19-Sep-2013); kestenova šuma (leg. Janković, $M$. 22-Jul-1957, det. M. Niketić, 40889 BEOU, sub Viola sp., rev. M. Niketić, 07-Sep-2013); kestenove šume (leg. Janković, M. 08-May-1960, det. M. Niketić, 40888 BEOU, sub Viola sp.); manastir Dečani, UTM DN31, Exp. S, 750 m, kestenove šume (leg. Janković, M. 29-May-1959, det. M. Niketić, 40794 BEOU, sub V. sylvestris); Lumbardska planina, UTM DN22, DN32, Exp. S, munikova šuma (leg. Janković, M. 22-May-1959, 40796 BEOU, rev. M. Niketić, 19-Sep-2013); Peć, Lumbardska planina, UTM DN22, DN32 (leg. Janković, M. 23-May-1959, det. M. Niketić, 40842 BEOU, sub Viola sp.); Rugovska klisura, UTM DN22, DN32 (leg. Rudski, I. 27-Apr-1937, det. M. Niketić, BEO, sub Viola sp.); Prilepska planina, Raški do, UTM DN22, Exp. NW, požarište, bukovo-jelove šume, 04-Jun1959, det. M. Niketić, 40849 BEOU); Raški do-Savine vode-Amšor-Žljeb-Kovraga, UTM DN21, DN22 (leg. Janković, M. 28-May-07-Jun-1960, det. M. Niketić, 40867 BEOU, sub Viola sp.); Žljeb 
(Maja Rusolija), Lipa selo, iznad, UTM DN33, krečnjak (leg. Černjavski, P., Rudski, I., Lindtner, V. 01-Aug-1933, det. P. Černjavski, 8209 BEO); Šar-planina: Velibeg-Vraca, UTM DM73 (leg. Džukić, G. 26-Aug-1995, det. M. Niketić, 2872-96 BEOU-KEGB, sub Viola sp.).

Viola rupestris F. W. Schmidt (Fig. 13)

Banat Deliblatska peščara (leg. Grebenščikov, O., 06-Apr-1947, det. M. Niketić, 1240 BEO, sub Viola sp.); Čardak-Vrelo, UTM EQ17, pesak, Aceri tatarici-Quercion (leg. Stevanović, V. 13-Apr-1997, 4851 BEOU-KEGB, sub $V$. suavis, rev. M. Niketić, 07-Sep-2013); Deliblato, UTM EQ06, pesak, peščara (leg. Soška, Th. 08-May-1922, 45114 BEOU); Dolovo, UTM DQ97, bagremova šuma (leg. Stevanović, V. 10-Apr-1970, 1303 BEOU-KEGB, sub V. suavis, rev. M. Niketić, 13-Sep-2013); (leg. Stevanović, V. 10-Apr-1970, det. M. Niketić, 1292 BEOU-KEGB, sub Viola sp.); E Serbia Rtanj, UTM EP64, EP74 (leg. Pančić, J. 01Apr-1869, det. J. Pančić, 3215 BEOU (Herb. Panc.), sub $V$. arenaria, rev. M. Niketić, 21Sep-2013); Suva planina, 41059 BEOU, rev. M. Niketić, 13-Sep-2013); Devojački grobTrem, UTM EN98, krečnjak (leg. Tomović, G. 23-May-2005, det. M. Niketić, 19824 BEOUKEGB, sub Viola sp.); Pomoravlje Veliko Gradište: Ram, Ramska peščara, UTM EQ25, EQ26 (leg. Bogojević, R., 10072 BEOUKEGB, sub $V$. sylvestris, rev. M. Niketić, 13Sep-2013); SW Serbia Golija, UTM DN49, det. M. Niketić, 41024 BEOU).

Viola stagnina Kit. (Fig. 14) Šumadija Beograd: Makiš, UTM DQ55 (leg. Pančić, J., 01-Apr-1880, det. J. Pančić, 3274 BEOU (Herb. Panc.), sub V. pratensis, rev. M. Niketić, 21-Sep-2013); (leg. Soška, Th. Jun-1929, 45063 BEOU, sub V. elatior, rev. M. Niketić, 07-Sep-2013); Beli Potok, UTM DQ65, po bujnim livadama (leg. Pančić, J. 01-Jun1875, det. J. Pančić, 14999 BEOU (Herb. Panc.), rev. M. Niketić, 07-Sep-2013); vlažna livada (leg. Rajevski, L. 10-Apr-1937, 1507 BEO, sub $V$. pumila, rev. M. Niketić, 25-Sep2013); Žarkovo, UTM DQ55 (leg. Lindtner, V. May-1936, det. V. Lindtner, 1510 BEO, sub $V$. pumila, rev. M. Niketić, 25-Sep-2013); (leg. Lindtner, V. 21-Apr-1953, det. V. Lindtner, 1506 BEO, sub $V$. pumila, rev. M. Niketić, 25Sep-2013); Knić, UTM DP76 (leg. Lindtner,

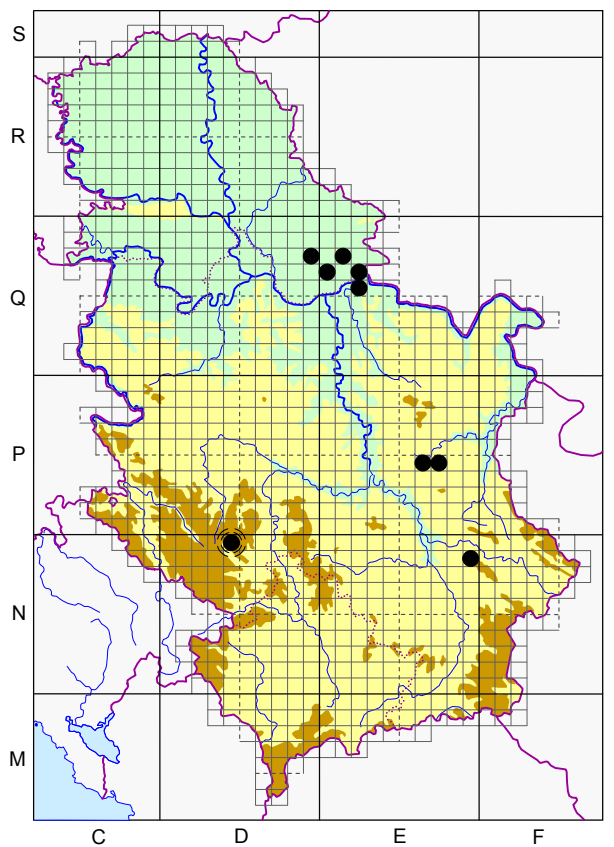

Fig. 13. - Distribution of the species $V$. rupestris F. W. Schmidt in Serbia.

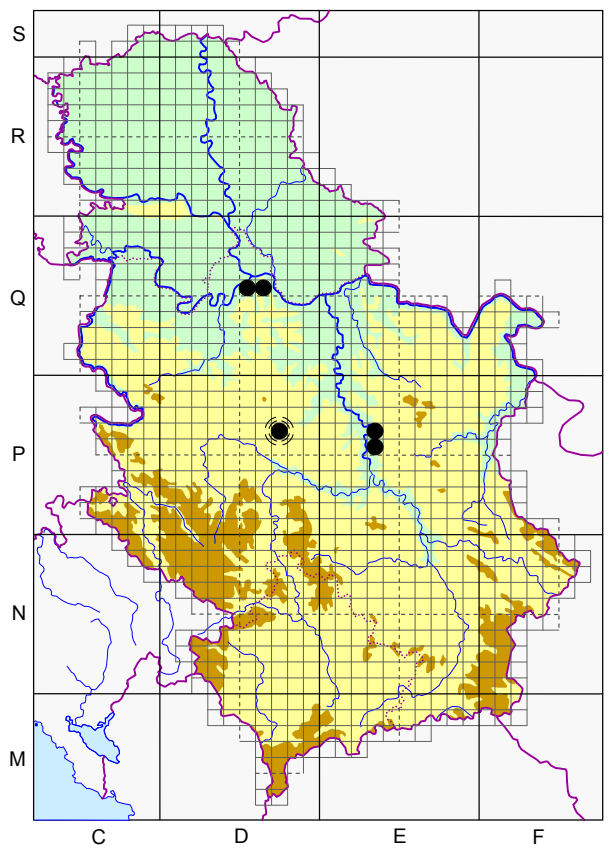

Fig. 14. - Distribution of the species $V$. stagnina Kit. in Serbia. 
V. 22-Jun-1949, det. V. Lindtner, 8248 BEO, sub V. elatior, rev. M. Niketić, 25-Sep-2013); Pomoravlje Ćuprija-Paraćin, UTM EP35, EP36 (leg. Lindtner, V., 30-May-1950, det. V. Lindtner, 8247 BEO, sub V. elatior, rev. M. Niketić, 25-Sep-2013).

Viola suavis Bieb. (Fig. 15)

Banat Deliblatska peščara: Čardak-VreIo, UTM EQ17, pesak, Aceri tatarici-Quercion (leg. Stevanović, V., 13-Apr-1997, 4851 BEOUKEGB, rev. M. Niketić, 07-Sep-2013); Deliblato, UTM EQ06 (leg. Stevanović, V., 1186 BEOUKEGB); Zrenjanin: Melenci, banja Rusanda, UTM DR44, slatine, pod kulturom crnog bora (leg. Nikolić, V., Diklić, N. 24-May-1973, 43096 BEO); Srem Fruška gora: Iriški venac, UTM DR00, mešovite listopadne šume (leg. Nikolić, V., Diklić, N., 06-May-1971, det. N. Diklić, 42753 BEO, sub V. alba, rev. M. Niketić, 25-Sep-2013); Slankamen, Koševac, UTM DR30, Exp. N, 244 m, les, stepski fragmenti (leg. Niketić, M., Tomović, G. 13-Apr2014, det. M. Niketić, BEO); NW Serbia Gučevo, UTM CQ52, krečnjak (leg. Niketić, M., Tomović, G., 01-Nov-2008, det. M. Niketić, BEO); Podrinje: Sokolska planina, Soko grad, UTM CQ70, dolomit (leg. Niketić, M. 29Apr-2010, det. M. Niketić, BEO, sub V. hirta); krečnjak (leg. Niketić, M. 29-Apr-2010, det. M. Niketić, BEO); Valjevo: Gradac reka kanjon, $1,5 \mathrm{~km}$ uzvodno od man. Ćelije, UTM DP09, $250 \mathrm{~m}$, krečnjak, šikare (leg. Niketić, $M$. 25-Apr-1992, det. M. Niketić, BEO); Valjevo-

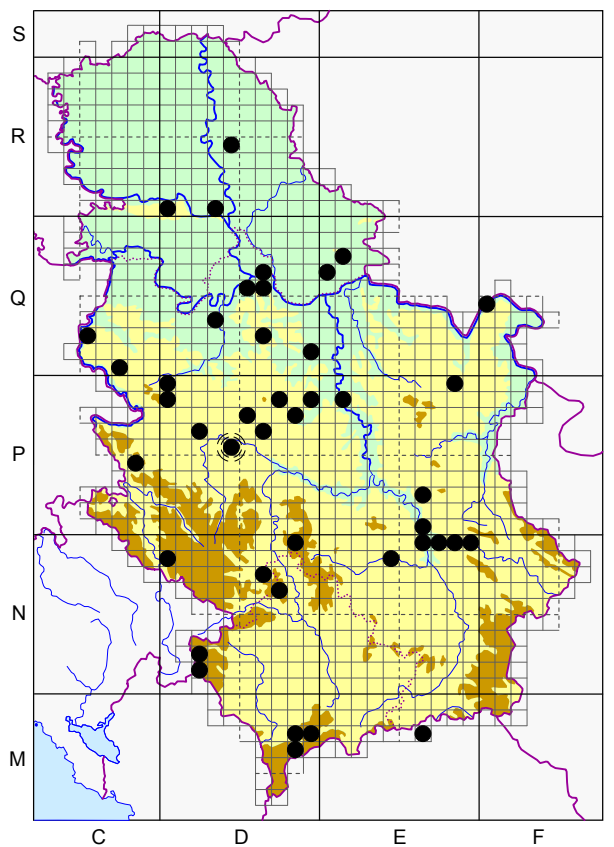

Fig. 15. - Distribution of the species V. suavis Bieb. in Serbia.

Kosjerić: Taorska Vrela, UTM DP08, krečnjak, stene (leg. Ostojić, D. 12-Sep-1997, 7515 BEOUKEGB, sub V. alba, rev. M. Niketić, 21-Sep-2013); Šumadija Beograd: Avala, Beli Potok, UTM DQ65, bukova šuma (leg. Nikolić, V., 01-Apr-1953, det. V. Nikolić, 1543 BEO, sub V. odorata, rev. M. Niketić, 25-Sep-2013); Košutnjak, UTM DQ55, 50 m, šuma (leg. Jovanović, P. 18-Apr-2007, 28407 BEOU-KEGB, sub $V$. odorata, rev. M. Niketić, 21-Sep-2013); hrastova izdanačka šuma (leg. Rajevski, $L$. 16-Mar-1938, 1529 BEO, sub V. sepincola, rev. M. Niketić, 25-Sep-2013); (01-Apr-1926, det. M. Niketić, 40883 BEOU); (leg. Grebenščikov, O. 03-Jul-1943, det. P. Černjavski, Th. Soška, 1519 BEO, sub V. riviniana, rev. M. Niketić, 25-Sep-2013); (leg. Janković, M. Apr-1953, det. M. Niketić, 40884 BEOU, sub Viola sp.); (leg. Lakušić, D. 31-Mar-1986, 947-86, 948-86 BEOU-KEGB, sub V. alba, rev. M. Niketić, 21-Sep-2013); (leg. Niketić, M. 03-Oct-2008, det. M. Niketić, BEO); Rakovica, UTM DQ55, hrastova izdanačka šuma (leg. Rajevski, L. 25-Oct-1938, 1526 BEO, sub V. sepincola, rev. M. Niketić, 25-Sep2013); Veliko selo, UTM DQ66 (leg. Vukojičić, S., Tomović, G., Lazarević, P. 03-Apr-2003, 16330 BEOUKEGB, sub V. alba, rev. M. Niketić, 21-Sep-2013); Višnjica, UTM DQ66 (leg. Borisavljević, Lj. 23-Mar1950, det. Lj. Borisavljević, 1534 BEO, sub V. alba, rev. M. Niketić, 25-Sep-2013); (leg. Niketić, M. 08Apr-2006, det. M. Niketić, BEO); (leg. Soška, Th. Apr-1914, 40715 BEOU); (leg. Soška, Th. Apr-1914, 45150 BEOU, sub V. pontica, rev. M. Niketić, 07-Sep-2013); Bela Stena, Gradina, UTM DQ66, 130 $\mathrm{m}$, les (leg. Niketić, M. 22-May-2005, det. M. Niketić, BEO); Duboki dol, UTM DQ66, 01-Apr-1926, 40882 BEOU, rev. M. Niketić, 07-Sep-2013); Lipak, UTM DQ66, šuma (leg. Bogojević, R. 24-May-1960, 40758 BEOU, sub V. alba, rev. M. Niketić, Apr-2005); Gornji Milanovac: Brđanska klisura, Brđani, UTM DP57, po krševima (leg. Pančić, J. 01-Jul-1852, det. J. Pančić, 3265 BEOU (Herb. Panc.), sub V. odorata, rev. M. Niketić, 07-Sep-2013); Knić: Borač, UTM DP66, silikat (leg. Niketić, M., Tomović, G. 17-May-2004, det. M. Niketić, BEO); Kragujevac: Dobrača, Seničko polje, UTM 
DP78 (leg. Rudski, I. 24-Apr-1938, det. M. Niketić, 1296 BEO, sub Viola sp.); Drača, UTM DP87 (leg. Rudski, I. 06-Jun-1980, det. M. Niketić, 1273 BEO, sub Viola sp.); Resnik, UTM DP98 (leg. Rudski, I. 06-May-1939, 8148 BEO, sub V. sepincola, rev. M. Niketić, 25-Sep-2013); Mladenovac: Kosmaj, Košutica, UTM DQ62 (leg. Niketić, M., Tomović, G. 10-May-2003, det. M. Niketić, BEO); Veliki Kosmaj, UTM DQ62, silikat (leg. Niketić, M., Tomović, G. 10-May-2003, det. M. Niketić, BEO); Obrenovac: Veliko polje - Čekićeva šuma, UTM DQ33, Querco-Carpinetum (leg. Stanojević, S. 25-Apr-1996, det. S. Stanojević, 4907 BEOU-KEGB, sub V. odorata, rev. M. Niketić, 21-Sep-2013); Pomoravlje Smederevska Palanka (leg. Pečnik, 1943, det. P. Černjavski, 8144 BEO, sub V. sepincola, rev. M. Niketić, 25-Sep-2013); Glibovac, UTM DQ91, voćnjak (leg. Tomić, A. 24-Mar-1992, det. A. Tomić, 41071 BEOU, sub $V$. odorata, rev. M. Niketić, 07-Sep-2013); Velika Morava: Bagrdan klisura, Brzan, UTM EP18, silikat (leg. Niketić, M. 09-Apr-2010, det. M. Niketić, BEO, sub V. hirta); NE Serbia Bor: Veliki krš, glavni vis, UTM EP89, šibljak (leg. Diklić, N., 29-Jun-1960, det. M. Janković, 43097 BEO); Đerdapska klisura: Kazan, UTM FQ04, krečnjak (leg. Niketić, M., Tomović, G. 14-Apr-2008, det. M. Niketić, BEO); W Serbia Čačak, UTM DP45 (leg. S. P., det. M. Niketić, 41067 BEOU, sub Viola sp.); Mokra gora: Krsmanski potok, UTM CP84 (leg. Soška, Th. Jun-1914, 45141 BEOU, sub V. odorata, rev. M. Niketić, 07-Sep-2013); C Serbia Goč: Gvozdac, Jelen Do, UTM DP26, krečnjak (leg. Niketić, M., Tomović, G., 11-Apr-2009, det. M. Niketić, BEO, sub $V$. alba); Kopaonik: Brzeće, po hrptu grebena, UTM DN89, Exp. SE, 1300 m, Apr-2005, 40787 BEOU, rev. M. Niketić, 07-Sep-2013); Metođe, Lisičje stene, UTM DN89, 1600 m, krečnjak, stene (leg. Lakušić, D., Niketić, M. 18-Sep-1986, det. D. Lakušić, 40797 BEOU, sub V. hirta, rev. M. Niketić, 07Sep-2013); Prokuplje: Gubetin, UTM EN48, silikat (leg. Niketić, M., Tomović, G. 30-Apr-2008, det. M. Niketić, BEO); E Serbia Niš: Gabrovac, Del - Milljkov del, UTM EN79, 420 m, kompozitna podloga, šikare (leg. Niketić, M., 27-Oct-2013, det. M. Niketić, BEO); Jelašnička klisura, UTM EN89 (leg. Stevanović, V., Niketić, M., Tomović, G. 15-Mar-1998, det. M. Niketić, 10942 BEOU-KEGB, sub Viola sp.); Radovanski kamen, UTM EN89, 580 m, krečnjak, Carpinetum orientalis (leg. Niketić, M., Buzurović, U. 07-May-2014, det. M. Niketić, BEO); Krušce, UTM EN69, 50 m, les, šikare (leg. Savić, A. 02-Apr-2007, 28373 BEOU-KEGB, sub $V$. odorata, rev. M. Niketić, 21-Sep-2013); okolina (leg. Petrović, S. 1879, det. W. Becker, 45095 BEOU, sub V. collina, rev. M. Niketić, 07-Sep-2013); Paljina, leva obala Toponičke reke, UTM EP60, 200 m, aluvijalni nanosi, mezofilne livade (leg. Niketić, M. 14Oct-2013, det. M. Niketić, BEO); Sićevačka klisura, Oblik, UTM EN99, Carpinetum orientalis (leg. Niketić, M. 07-Apr-2007, det. M. Niketić, BEO); (leg. Niketić, M. 09-May-2007, det. M. Niketić, BEO); krečnjak, Carpinetum orientalis (leg. Niketić, M. 28-May-2007, det. M. Niketić, BEO); Suvi do, Dubrava, UTM EN79, 300 m, kompozitna podloga, bagremova šuma (leg. Niketić, M. 29-Oct-2013, det. M. Niketić, BEO); Ozren: Janior, UTM EP62 (leg. Antonijević, R. 07-May-1957, det. M. Niketić, 41070 BEOU, sub Viola sp.); Svrljiške planine: Krupac, istočno od sela, UTM EN99, Exp. N, 400 m, krečnjak, kamenjari i stene (leg. Niketić, M. 01-Mar-2014, det. M. Niketić, BEO); SW Serbia Giljeva: Krajinovići, Čelo brdo, UTM DN08, Exp. NW, 1000 m, serpentinit (leg. Niketić, M., Tomović, G., Stevanović, V., Vukojičić, S., 28-Apr-2006, det. M. Niketić, BEO, sub V. hirta); Rogozna: BanjaGrižani Han, UTM DN67, DN76 (leg. Soška, Th. 23-Jun-1914, 40745 BEOU, sub V. pontica, rev. M. Niketić, 01-Oct-2013); Grižani Han, UTM DN76 (leg. Soška, Th. Jun-1914, det. W. Becker, 45223 BEOU, sub V. odorata, rev. M. Niketić, 01-Oct-2013); S Serbia Rujan planina: Veliki Orljak, UTM EM67, krečnjak (leg. Niketić, M., Tomović, G., 04-Apr-2010, det. M. Niketić, BEO; BEO, sub V. odorata); Kosovo Šar-planina: Ošljak, UTM DM87, DM97 (leg. Rudski, I., 20-Jul-1930, 45067 BEOU, sub V. pontica, rev. M. Niketić, 07-Sep-2013); Gornje selo, UTM DM97 (leg. Rudski, I. 18-Jul1930, 45072 BEOU, sub V. pontica, rev. M. Niketić, 07-Sep-2013); Mušnikovo, UTM DM97 (leg. Rudski, I. 09-Jul-1930, 45070 BEOU, sub V. pontica, rev. M. Niketić, 07-Sep-2013); Metohija Prizren: Prizrenska Bistrica - klisura, UTM DM87 (leg. Soška, Th., 10-Jul-1914, 40748 BEOU, sub V. pontica, rev. M. Niketić, 07-Sep-2013); Prokletije: Prilepska planina, Raški do-Savine vodeAmšor-Žljeb-Kovraga, UTM DN21, DN22 (leg. Janković, M. 28-May-07-Jun-1960, det. M. Niketić, 40868 BEOU, sub Viola sp.); Šar-planina: Ljubinje, Ljubinjski potok, UTM DM86, 04-Jul-1930, det. M. Niketić, 45066 BEOU); Nebregošte, UTM DM86 (leg. Rudski, I. 02-Jul-1930, 45069 BEOU, sub $V$. pontica, rev. M. Niketić, 07-Sep-2013). 


\section{V. sect. DISCHIDIUM Ging.}

Viola biflora L. (Fig. 16)

W Serbia Tara planina: Beli Rzav - klisura, Sklopovi (Zaovine-Kotroman), UTM CP75, krečnjak, vlazne stene uz reku (leg. Zlatković, B., Ostojić, D., det. B. Zlatković, 16034 BEOU); $\underline{\text { C }}$ Serbia Kopaonik, smrčeva šuma (leg. Đelfi, B., 25-May-1936, det. B. Đelfi, 8273 BEO); subalpska smrča (leg. Popović, M.? 26-Jun-1954, det. G. Tomović, 41943 BEOU); (leg. Pančić, J. 01-Aug-1852, det. J. Pančić, 3218 BEOU (Herb. Panc.)); (leg. Pančić, J. 1863, det. J. Pančić, 3219 BEOU (Herb. Panc.)); (leg. Smerečinska, J. 20May-1936, det. J. Smerečinska, 8269 BEO); Brzećka reka, UTM DN89 (leg. Lakušić, D., Mitrović, V., "ENDEMIT" 27-May-2001, det. D. Lakušić, 13925 BEOU-KEGB); Brzećka reka - dolina, UTM DN89 (leg. Lakušić, D. 27-May2001, det. D. Lakušić, 11009 BEOU-KEGB); Gobelja, Mala Gobelja, UTM DN89, Exp. $\mathrm{N}, 1720$ m, kamenjar na granici smrševe šume, 11-Jun-1952, det. G. Tomović, 41946 BEOU); Oštri krš, UTM DN89, 1700 m, krečnjak, Piceetum excelsae (leg. Nikolić, D. 15-Jun-1988, det. D. Lakušić, 41948 BEOU); Jadovnik, UTM DN79 (leg. Pančić, J. 01-Jul-1864, det. J. Pančić, 3180 BEOU (Herb. Panc.)); Kadije-

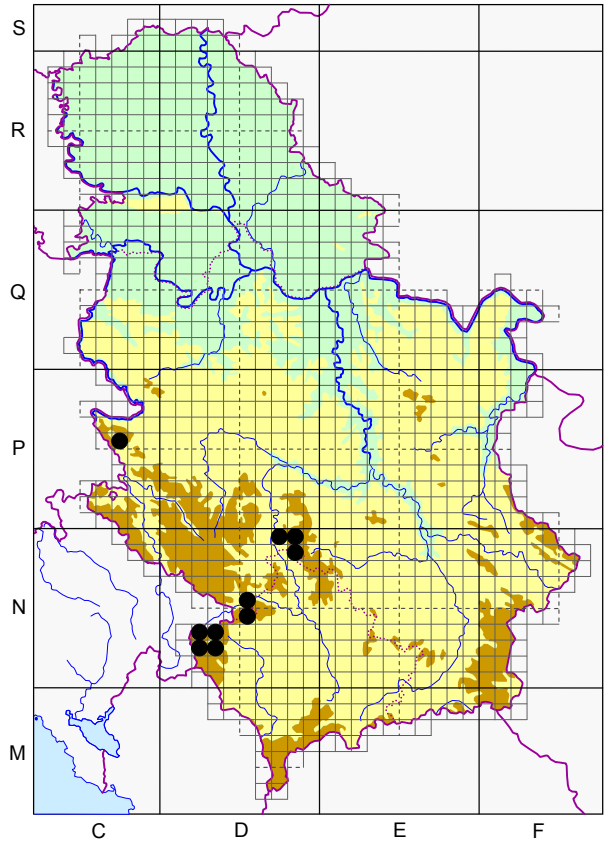

Fig. 16. - Distribution of the species $V$. biflora L. in Serbia.

vac, Hajdučki potok, kod Strugare, UTM DN89, 1500 m, Piceetum excelsae (leg. Stanić, S. et al. 14Jul-1988, det. D. Lakušić, 41949 BEOU); Karaman, Mali Karaman, UTM DN89, 1850 m, granit, Vaccinio-Juniperetum nanae (leg. Lakušić, D. 07-Jun-1988, det. D. Lakušić, 41950 BEOU); Vučak, UTM DN89, 1600-1700 m, smrčeva šuma, 18-Jun-1952, det. G. Tomović, 41947 BEOU); Krčmar, UTM DN89 (leg. Rudski, I. 10-Jul-1938, det. I. Rudski, 8268 BEO); Samokovska reka, iznad, UTM DN89, smrčeva šuma sa mahovinom, 09-Jun-1952, det. G. Tomović, 41945 BEOU); Samokovska reka dolina, UTM DN89, tresava (leg. Popović, M.? 21-Jun-1954, det. G. Tomović, 41944 BEOU); Samokovska reka - klisura, UTM DN89 (leg. Stevanović, V., Niketić, M. Jun-1985, det. D. Lakušić, 41952 BEOU); Suvo rudište, UTM DN89, Exp. N, 1700 m, granit, Piceetum excelsae (leg. Lakušić, D. 05Jul-1991, det. D. Lakušić, 41951 BEOU); Suvo rudište-Nebeske stolice, UTM DN88, DN89, 1800 m, silikat, ivica smrčeve šume (leg. Lakušić, D. 16-Jun-2007, det. D. Lakušić, 24622 BEOU-KEGB); SW Serbia Stari Kolašin: Batrage, Draška suhovara, UTM DN55, smrčeva šuma (leg. Rudski, I., 06-Jul-1932, det. S. Vukojičić, BEO); Metohija Mokra gora: Novin vrh, UTM DN54, smrčeva šuma (leg. Rudski, I., 06-Jul-1932, det. I. Rudski, BEO); Prokletije: Košutine, Košutina lokva, UTM DN23 (leg. Gošović, S. 10-Jun-1922, det. S. Gošović, 41939 BEOU); Lumbardska planina, UTM DN22, DN32, Exp. N, munikova šuma (leg. Janković, M. 23-May-1959, det. M. Janković, 41941 BEOU); Prilepska planina, Raški do, UTM DN22, Exp. NW, požarište bukovo-jelove šume (leg. Janković, M. 04-Jun-1959, det. S. Vukojičić, 41942 BEOU); Žljeb (Maja Rusolija), UTM DN33, 1900 m (leg. Rudski, I. 25-Jun-1932, det. S. Vukojičić, BEO); 2000 m (leg. Rudski, I. 25-Jun-1932, det. I. Rudski, BEO); 2100 m (leg. Rudski, I. 25-Jun-1932, det. S. Vukojičić, BEO); Exp. NE, 2272 m, krečnjak (leg. Černjavski, P., Rudski, I., Lindtner, V. 02-Aug-1933, det. P. Černjavski, 8271 BEO). 


\section{V. sect. MeLANIUM Ging.}

Viola aetolica Boiss. \& Heldr. (Fig. 17)

Metohija Koritnik, UTM DM65, DM66 (leg. Oehm, H., May-1937, 8506 BEO, sub V. saxatilis subsp. aetolica, rev. G. Tomović, 21-Dec2012); Mokra gora, UTM DN54 (leg. Petrović, D., 41298 BEOU, sub V. lutea, rev. G. Tomović, 21-Dec-2012); Paštrik, UTM DM67 (leg. Džukić, G., Milojević, B. 19-Jul-1980, det. G. Tomović, 1071, 1093, 1117 BEOU-KEGB); Prokletije: Bogićevica, Babino polje, UTM DN21, nekošene livade (leg. Černjavski, P., Rudski, I., Lindtner, V. 19-Jul-1933, det. P. Černjavski, 8450 BEO, sub $V$. elegantula var. latisepala, rev. G. Tomović, Dec-2012); smrčeva šuma (leg. Černjavski, P., Rudski, I., Lindtner, V. 19-Jul1933, det. P. Černjavski, 8472 BEO, sub $V$. elegantula var. latisepala, rev. G. Tomović, Dec-2012); Dobroška planina, UTM DN21 (leg. Černjavski, P., Rudski, I., Lindtner, V. 18-Jul1933, det. P. Černjavski, 8474 BEO, sub $V$. elegantula var. latisepala, rev. G. Tomović, Dec-2012); Tropojske pločice, UTM DN21, kamenjari (leg. Černjavski, P., Rudski, I., Lindtner, v. 18-Jul-1933, det. P. Černjavski, 8473 BEO, sub $V$. elegantula var. latisepala, rev. G. Tomović, Dec-2012); Koprivnik, UTM DN20, in

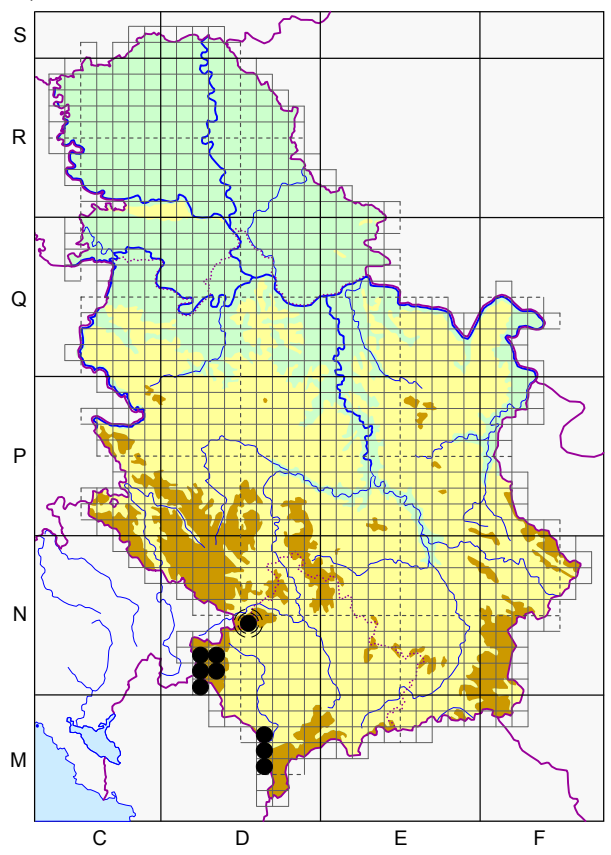

Fig. 17. - Distribution of the species $V$. aetolica Boiss. \& Heldr. in Serbia. mugeti (leg. Soška, Th. 15-Jun-1923, 41123

BEOU, sub V. alpestris subsp. aetolica, rev. G. Tomović, Apr-2007); in pascuis subalpinis (leg. Soška, Th. Jun-1923, 41121 BEOU, sub V. alpestris subsp. aetolica, rev. G. Tomović, Apr-2007); (leg. Soška, Th. 15-Jun-1923, 41122 BEOU, sub V. alpestris subsp. aetolica, rev. G. Tomović, Apr-2007); UTM DN31, DN32, 1500 m, in pratis (leg. Grebenščikov, O. 08-Jul-1939, det. G. Tomović, BEO); vlažni mugus (leg. Janković, M. 11-Jul-1963, det. M. Janković, 41293 BEOU, sub V. elegantula?, rev. G. Tomović, 21-Dec2012); Kurvala, Ločanska planina, UTM DN31, suvati iznad šumskog pojasa (leg. Černjavski, P., Rudski, I., Lindtner, V. 15-Jul-1933, det. P. Černjavski, 8470 BEO, sub V. elegantula var. latisepala, rev. G. Tomović, Dec-2012); Pločica, UTM DN21, DN31, 2100-2200 m, škriljci (leg. Černjavski, P., Rudski, I., Lindtner, V. 16-Jul-1933, det. P. Černjavski, 8465 BEO, sub V. elegantula var. latisepala, rev. G. Tomović, Dec-2012); (leg. Lindtner, V. 1933, det. G. Tomović, BEO); vrh, UTM DN31, livade (leg. Černjavski, P., Rudski, I., Lindtner, V. 16-Jul-1933, det. P. Černjavski, 8539 BEO, sub V. saxatilis, rev. G. Tomović, 23-Sep-2013); Lumbardska planina, UTM DN22, DN32, munikova šuma (leg. Janković, M. 22-May-1959, det. M. Janković, 41297 BEOU, sub V. saxatilis subsp. aetolica, rev. G. Tomović, 21Dec-2012); Nedžinat, UTM DN22, krečnjak, stene u borovoj šumi (leg. Černjavski, P., Rudski, I., Lindtner, V. 28-Jul-1933, det. P. Černjavski, 8466 BEO, sub V. elegantula var. latisepala, rev. G. Tomović, Dec-2012); pod vrhom, UTM DN22, krečnjak, stene (leg. Černjavski, P., Rudski, I., Lindtner, V. 28-Jul-1933, det. P. Černjavski, 8475 BEO, sub V. elegantula var. latisepala, rev. G. Tomović, Dec2012); Prilepska planina, Raški do-Savine vode-Amšor-Žljeb-Kovraga, UTM DN21, DN22 (leg. Janković, M. 28-May - 07-Jun-1960, det. M. Janković, 41295 BEOU, sub V. elegantula?, rev. G. Tomović, 21-Dec-2012); Rugovska klisura, Slane poljane, UTM DN32, munikova šuma (leg. Janković, M. 06-Jul-1960, det. M. Janković, 41294 BEOU, sub V. elegantula?, rev. G. Tomović, 21-Dec-2012). 
Viola arvensis Murray (Fig. 18) Bačka Subotičko-Horgoška peščara: Ludaško jezero, severozapadno od jezera, UTM DS00, les, pustare (leg. Niketić, M., Tomović, G., 12-Apr-2014, det. G. Tomović, BEO); Banat Deliblatska peščara (leg. Lakušić, D., 01-May-1991, 2890 BEOU-KEGB, rev. G. Tomović, 07-Sep-2013); (leg. Stevanović, V., det. V. Stevanović, 1387 BEOU-KEGB, sub $V$. kitaibeliana); Deliblato, UTM EQ06 (leg. Miljković, B. 1929, det. G. Tomović, VA-001 BEO, sub V. sylvestris); (leg. Soška, Th. May1929, det. G. Tomović, 41995 BEOU); Devojački bunar, UTM DQ98, $160 \mathrm{~m}$, peščarska vegetacija, Festucetea vaginatae (leg. Stevanović, V., Lakušić, D., Kabaš, E. 28-May-2011, det. G. Tomović, 37287 BEOU-KEGB); Flamunda, UTM EQ07 (leg. Niketić, M., Lakušić, D., Tomović, G. 10-May-1998, 10814 BEOU-KEGB, sub V. tricolor, rev. G. Tomović, 07-Sep-2013); (leg. Soška, Th. Apr-1943, det. Th. Soška, 8567 BEO, sub V. kitaibeliana, rev. G. Tomović, 23Sep-2013); Grebenac, UTM EQ17, kultura belog bora (leg. Stevanović, V. 30-May-1970, det. V. Stevanović, 1326 BEOU-KEGB, sub $V$. kitaibeliana, rev. G. Tomović, 07-Sep-2013); Korn bunar, UTM EQ07, peščara (leg. Stevanović, V., Jovanović, S. 05-May-1990, det. G. To-

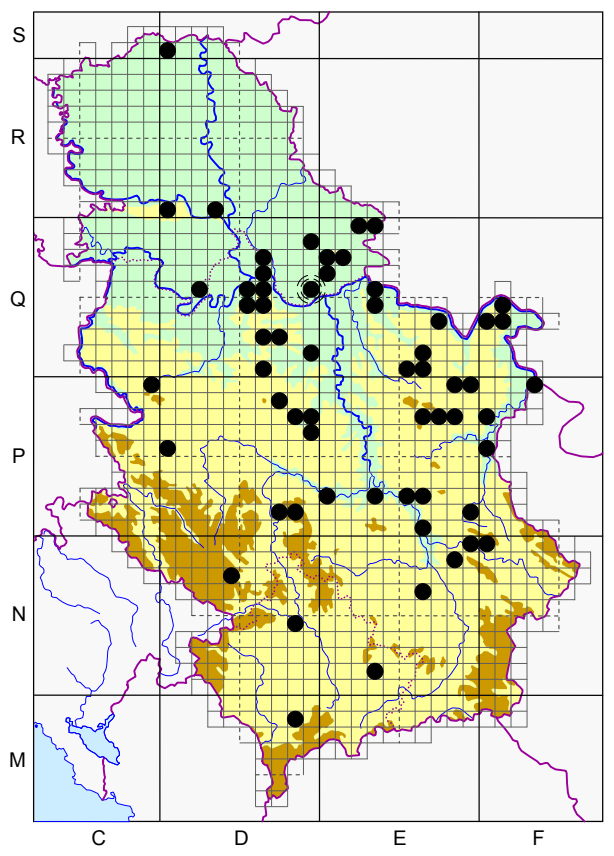

Fig. 18. - Distribution of the species $V$. arvensis Murray in Serbia. mović, 309-90 BEOU-KEGB, sub $V$. arvensis?); peščarska vegetacija (leg. Stevanović, V., Jovanović, $S$. 05-May-1990, det. G. Tomović, 309 BEOU-KEGB, sub V. arvensis?); Kovin, UTM DQ95 (leg. Jovanović, E. 01-May-2002, det. E. Jovanović, 41971 BEOU, sub V. tricolor, rev. G. Tomović, 07-Sep2013); Pančevo: Jabuka, Jabučki rit, UTM DQ67 (leg. Gajić, D. 15-May-1953, det. D. Gajić, 41978 BEOU, sub V. arvense); (leg. Gajić, D. 15-May-1953, det. G. Tomović, 41979 BEOU); Vršačke planine (leg. Nikolić, V., Diklić, N. 28-Apr-1975, det. G. Tomović, 42772 BEO); odmaralište "13. maj" - Iugarnica, UTM EQ29, šuma kitnjaka i lipe (leg. Nikolić, V., Diklić, N. 06-May-1970, det. N. Diklić, 42764 BEO, rev. G. Tomović, 23-Sep-2013); odmaralište "13. maj" - Velika Čoka, UTM EQ29, EQ39 (leg. Nikolić, V., Diklić, N. 16-Jul-1969, det. N. Diklić, 42818, 42819 BEO, sub V. arvensis var. gracilensis); planinarski dom Široko bilo, UTM EQ29, proplanci (leg. Nikolić, V., Diklić, N. 26-Jun1968, det. G. Tomović, 42768 BEO, sub V. arvensis?, rev. G. Tomović, 23-Sep-2013); Vršačka kula, UTM EQ29 (leg. Nikolić, V., Diklić, N. 27-Apr-1967, det. G. Tomović, 42808 BEO, sub V. banatica?, rev. G. Tomović, 23-Sep-2013); Srem Fruška Gora: Novi Slankamen, Vinogradi, kota 244, UTM DR30, Exp. S, 244 m, les, stepski fragment (leg. Niketić, M., Tomović, G., 13-Apr-2014, det. G. Tomović, BEO); Zmajevac, UTM DR00, šuma kitnjaka i lipe (leg. Nikolić, V., Diklić, N. 26-May-1970, det. N. Diklić, 42763 BEO, sub V. saxatilis, rev. G. Tomović, Feb-2007); (leg. Stevanović, V., Jovanović, S., Lakušić, D. 09-Jun-1997, det. G. Tomović, 5394 BEOU-KEGB); Pećinci: Obedska bara, UTM DQ25 (leg. Milovanović, D. 10-Jun-1947, det. D. Milovanović, VA-002 BEO, sub V. kitaibeliana, rev. G. Tomović, 23-Sep-2013); NW Serbia Jablanik, UTM CP99, Exp. E, obrađeno zemljište (leg. Diklić, N., 24-Apr-1955, det. N. Diklić, 42777 BEO); Medvednik, UTM CP99 (leg. Pančić, J. 1875, det. J. Pančić, 3243 BEOU (Herb. Panc.), sub V. lutea, rev. G. Tomović, 07-Sep-2013); Šumadija Aranđelovac: Venčac, UTM DQ60, hrastov šibljak (leg. Diklić, N., 11-Jun-1962, det. N. Diklić, 42931 BEO, rev. G. Tomović, Feb-2007); Beograd, ruderalis (leg. Milošević, S. 1990, det. S. Milošević, 41970 BEOU); (leg. Pančić, J., det. J. Pančić, 3320 BEOU (Herb. Panc.), sub V. tricolor var. arvensis, rev. G. Tomović, 07-Sep-2013); Avala, UTM DQ64, DQ65, hrastova šuma (leg. Rajevski, L. 08-Jun-1938, det. L. Rajevski, VA-008 BEO); (leg. Mišić, V. 31-May-1961, det. G. Tomović, 41999 BEOU); Beli potok, UTM 
DQ65 (leg. Pavlović 1875, det. Pavlović, 42002 BEOU, sub V. tricolor); Pinosava, UTM DQ64, DQ65 (leg. Gajić, D. 06-Jun-1956, det. G. Tomović, 41980 BEOU); Grocka, UTM DQ65, bašta (leg. Bartula, M. 01-May-1996, det. M. Bartula, 42006 BEOU); Karaburma, UTM DQ66, in agris (leg. Lindtner, V. Apr1939, det. V. Lindtner, VA-007 BEO); (leg. Lindtner, V. Apr-1939, det. V. Lindtner, BEO); Košutnjak, UTM DQ55 (leg. Rajevski, L. 28-May-1937, det. L. Rajevski, VA-005 BEO); okolina, vinogradi, u žitu (leg. Pavlović 1873, det. Pavlović, 42003 BEOU, sub V. tricolor, rev. G. Tomović, 07-Sep-2013); Resnik, UTM DQ55 (leg. Grebenščikov, O. 15-Apr-1940, det. O. Grebenščikov, VA-006 BEO); Ripanj, UTM DQ54 (leg. Soška, Th. Jun-1922, det. Th. Soška, 41997 BEOU); Kragujevac: Drača, UTM DP87 (leg. Rudski, I. 06-Jun-1939, det. I. Rudski, 8555 BEO); Molerovića livada, UTM DP96, 06May, det. G. Tomović, 8554 BEO); Stragari, Kotraža, brdo Karaula, UTM DP78, krečnjak (leg. Niketić, M., Jovanović, M. 06-May-2004, det. G. Tomović, BEO); Sušica, ulica Beogradska, UTM DP97, 190 m, pored trotoara (leg. Bogdanović, M. 21-May-1997, det. V. Stevanović, 41987 BEOU, sub V. tricolor, rev. G. Tomović, 07-Sep-2013); Šumarice, UTM DP87 (leg. Rudski, I. 06-May-1936, det. I. Rudski, 8581 BEO, sub V. tricolor, rev. G. Tomović, 23-Sep-2013); Mladenovac: Kosmaj, UTM DQ62 (leg. Diklić, N. Oct-1955, det. N. Diklić, VA-003 BEO, rev. G. Tomović, 23-Sep-2013); vojna kasarna, UTM DQ72, pored puta (leg. Anđelković, S. 15-Apr-2001, det. S. Anđelković, 41988 BEOU, rev. G. Tomović, 07-Sep-2013); Pomoravlje Velika Morava: Smederevska Palanka, pored pruge (leg. Kočović, K., 04-May-2002, det. K. Kočović, 41989 BEOU, rev. G. Tomović, 07-Sep-2013); (leg. Kočović, K. 14-Aug-2001, det. K. Kočović, 41990 BEOU, rev. G. Tomović, 07-Sep-2013); (leg. Pečnik 1943, det. P. Černjavski, 8561 BEO); Glibovac, UTM DQ91, pored pruge (leg. Tomić, A. 05-May1992, det. M. Niketić, 41972 BEOU); pored puta (leg. Tomić, A. 09-Jun-1991, det. M. Niketić, 41976 BEOU); Mikulja (brdo), UTM DQ91, 02-May-1943, det. G. Tomović, 41973 BEOU); Vlajića brdo, UTM DQ91, njive (leg. Pečnik 09-May-1942, det. P. Černjavski, 8568 BEO, sub V. kitaibeliana, rev. G. Tomović, 23-Sep-2013); Veliko Gradište: Srebrno jezero, UTM EQ35 (leg. Bogojević, R. 01-Apr1987, 10053 BEOU-KEGB, sub $V$. kitaibeliana, rev. G. Tomović, 07-Sep-2013); Topolovnik, Lipovača, UTM EQ34, les (leg. Niketić, M. 30-Apr-2010, det. G. Tomović, BEO); NE Serbia Bor: Brestovačka banja, UTM EP87, livade (leg. Nikolić, V., Diklić, N., Bogdanović, M., 21-Jun-1972, det. G. Tomović, 42798 BEO); (leg. Pančić, J. 1870, det. J. Pančić, 3211 BEOU (Herb. Panc.), sub V. agrestis); (leg. Pančić, J. 1876, det. G. Tomović, 15003 BEOU (Herb. Panc.)); (leg. Soška, Th. 28-Jun-1929, det. G. Tomović, 41199 BEOU); Stol, UTM EP99, krečnjak, livade (leg. Nikolić, V., Diklić, N., Mladenović, S. 15Jun-1979, det. G. Tomović, 42778 BEO); Veliki krš, UTM EP89, Exp. NW (leg. Diklić, N. 14-Jul-1959, det. N. Diklić, 42790 BEO); Đerdapska klisura: Golo brdo, UTM FQ14, livade (leg. Nikolić, V., Diklić, N. 08-Jun-1970, det. G. Tomović, 42795 BEO); (leg. Nikolić, V., Diklić, N. 18-Jun-1969, det. V. Nikolić, 42781 BEO); Miroč, UTM FQ03, livade (leg. Diklić, N. 16-May-1955, det. N. Diklić, 42789 BEO, rev. G. Tomović, 23-Sep-2013); Pesača, UTM EQ73, kamenjari (leg. Nikolić, V., Diklić, N. 22Apr-1968, det. V. Nikolić, 42794 BEO); Pesača-Bosman, UTM EQ73, šuma (leg. Nikolić, V., Diklić, N. 21-Apr-1968, det. V. Nikolić, 42793 BEO); Tekija, UTM FQ14, Chrysopogonetum grylli (leg. Nikolić, V., Diklić, N. 16-Jun-1969, det. V. Nikolić, 42782 BEO); Kladovo: Podvrška, Velika čuka, UTM FQ13, krečnjak, stene (leg. Stevanović, V., Niketić, M., Tomović, G. 15-Jun-1998, 12363 BEOU-KEGB, sub V. arvensis?, rev. G. Tomović, 07-Sep-2013); Majdanpek: Majdanpečka Domena, Breza, UTM EQ61, krečnjak, pukotine stena, 18-Jul-1947, BEO, sub V. tricolor, rev. G. Tomović); livade (leg. Gajić, M., det. G. Tomović, VA-004 BEO); Malinik: Lazareva reka - kanjon, UTM EP67, EP77, krečnjak (leg. Lakušić, D., Tomović, G. 18-May-2007, det. G. Tomović, 19729 BEOU-KEGB); krečnjak, stene i kamenjari (leg. Stevanović, V. 18-May-1990, det. V. Stevanović, 358-90 BEOU-KEGB, sub V. arvensis \& tricolor, rev. G. Tomović, 07-Sep-2013); pored puta, 11-Jul-1985, det. G. Tomović, VA-001 BEOUKEGB); Negotin: Radujevac, Kapu Đalu, UTM FP39 (leg. Soška, Th. 31-Apr-1932, det. G. Tomović, 41993 BEOU); Zaječar: Timok reka - klisura, Čokonjar, Čokonjarsko brdo, UTM FP07, krečnjak (leg. Niketić, M., Tomović, G. 01-Jun-2005, det. G. Tomović, BEO); Vrška čuka, greben-vrh, UTM FP05, 650 m, krečnjak (leg. Nikolić, V., Diklić, N. 24-Jun-1975, det. G. Tomović, 42803 BEO); Žagubica: Milatovac-Vukovac, UTM EQ50, EQ60 (leg. Lindtner, V. 25-Jun-1951, det. N. Diklić, 42783 BEO); W Serbia Užice: Đetina reka - klisura, Stari grad, UTM DP05, krečnjak, in saxosis (leg. Soška, Th., Jun-1926, det. Th. Soška, 41994 BEOU); Zlatibor (leg. Pančić, J. 1875, det. J. Pančić, 3242 BEOU (Herb. Panc.), sub V. lutea, rev. G. Tomović, 07-Sep-2013); C Serbia Ibarska dolina: Ušće, Žaračka planina, Gokčanica selo, bliu, UTM DP71, 700-800 m (leg. Stevanović, V., Niketić, M., Vukojičić, S., Tomović, G., 02-May-2004, det. G. Tomović, BEO); Kruševac: Gaglovo, 
Sekljivica, UTM EP32, strnjište (leg. Bogdanović, M. 10-Aug-1971, det. G. Tomović, 42799, 42800, 42801 BEO); (42802 BEO, sub $V$. arvensis?); Trstenik: Bučje, Pečeni grob, UTM EP02, silikat, kamenjari (leg. Spasojević, J. 20-Apr-1992, det. D. Lakušić, 41998 BEOU); Željin, UTM DP81, serpentinit (leg. Stevanović, V. 23-Jun-1989, det. G. Tomović, 4189 BEOU-KEGB); E Serbia Aleksinac (leg. Pančić, J., 1868, det. J. Pančić, 3304 BEOU (Herb. Panc.), sub V. tricolor); Kraljevo, UTM EP52, vlažne dolinske livade (leg. Nikolić, V., Diklić, N. 13-Jun-1976, det. G. Tomović, 42779 BEO); Knjaževac: Svrljiška Topla, UTM EP91, krečnjak, stepski fragment (leg. Niketić, M., Tomović, G. 06Apr-2008, 26994 BEOU-KEGB, rev. G. Tomović, 07-Sep-2013); Niš: Mezgraja, UTM EP60, 21-May1955, det. G. Tomović, 41209 BEOU); Sićevačka klisura, manastir Sveta Petka, UTM EN99 (leg. Soška, Th. 04-Jun-1932, det. Th. Soška, 41991 BEOU); Sićevo, UTM EN88 (leg. Pančić, J. 1880, det. G. Tomović, 3203, 3204 BEOU (Herb. Panc.)); Ozren: Janior, UTM EP62 (leg. Diklić, N. 19-May-1957, det. N. Diklić, 42791 BEO); Svrljiške planine: Zeleni vrh, UTM FN09, 02-Jun-2008, det. G. Tomović, BEO); SW Serbia Novi Pazar: Sopoćani manastir, Careva česma, iznad, UTM DN47, krečnjak (leg. Košanin, N., det. G. Tomović, 41959 BEOU); vrelo Raške, UTM DN47, det. G. Tomović, 41975 BEOU); S Serbia Bojnik: Kacabać, UTM EN66, 250 m, les, vinogradi i voćnjaci (leg. Stojanović, D., 19-Apr-2009, 29447 BEOU-KEGB); Kosovo Gnjilane: Novo brdo, UTM EN31 (leg. Tomović, G., Ranđelović, V., Zlatković, B., 08-Aug-1997, det. G. Tomović, 8146 BEOU-KEGB); Šarplanina: Kodža Balkan, Rusenica reka - klisura, manastir Sveta Trojica, UTM DM88 (leg. Košanin, N. 06-Jun-1923, det. N. Košanin, 41984 BEOU); Zvečan, UTM DN84 (leg. Košanin, N. 18-Apr1913, det. N. Košanin, 41985 BEOU).

Viola calcarata subsp. zoysii (Wulfen) Merxm. (Fig. 19)

Metohija Koritnik: Rapča, UTM DM65, pojas bukve i munike (leg. Nikolić, V., Diklić, N., 12-Jul-1968, det. N. Diklić, 43183 BEO, sub $V$. zoysii); Ravnište, plato, UTM DM66, Nardetum (leg. Nikolić, V., Diklić, N. 28-Jul-1968, det. N. Diklić, 43184 BEO, sub V. zoysii); Prokletije: Bogićevica, UTM DN21, in saxosis (leg. Lindtner, V. 19-Jul-1933, det. P. Černjavski, 8304 BEO, sub $V$. zoysii); Babino polje, UTM DN21, krečnjak, in pratis alpinis (leg. Černjavski, P., Rudski, I., Lindtner, V. 17-Jul-1933, det. P. Černjavski, 8301 BEO, sub $V$. zoysii); Derviš Kom, UTM DN21, 2200-2250 m, granit (leg. Černjavski, P., Rudski, I., Lindtner, V. 17-Jul-1933, det. P. Černjavski, 8305 BEO, sub $V$. zoysii); 2200-2300 m, krečnjak, stene (leg. Černjavski, P., Rudski, I., Lindtner, V. 17-Jul-1933, det. G. Tomović, VZ-005 BEO, sub V. calcarata subsp. zoysii); Derviš Kom-Pločica, UTM DN21, krečnjak (leg. Černjavski, P., Rudski, I., Lindtner, V. 17-Jul-1933, det. P. Černjavski, 8306 BEO, sub V. zoysii); Koprivnik, UTM DN31, DN32, $2400 \mathrm{~m}$, in pascuis glareosis (leg. Grebenščikov, O. 10-Jul-1935, det. O. Grebenščikov, VZ-003 BEO, sub V. zoysii); ad nives deliquescentes (leg. Košanin, N. 15. Jun-1923, det. N. Košanin, 41450 BEOU, sub $V$. calcarata subsp. zoysii); ad nives lignescentis regioni mugheti (leg.

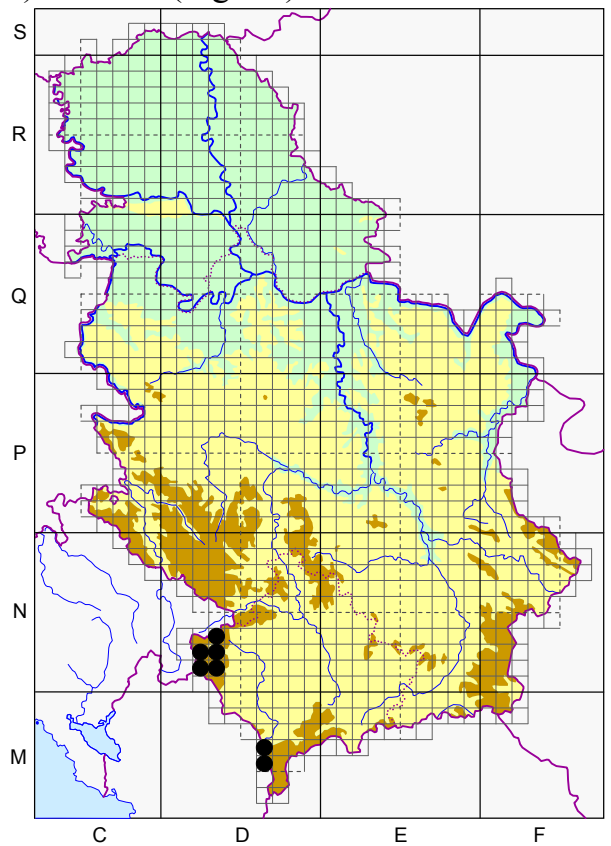

Fig. 19. - Distribution of the subspecies V. calcarata subsp. zoysii (Wulfen) Merxm. in Serbia. Soška, Th. 15-Jun-1915, det. Th. Soška, 41448 BEOU, sub V. zoysii); zona Pinus mugus, pored snega koji se topi (leg. Soška, Th. 15. Jun-1923, det. Th. Soška, 41449 BEOU, sub V. calcarata subsp. zoysii); (leg. Sobrijevski 1933, det. P. Černjavski, 8307 BEO, sub V. zoysii); Veternik, UTM DN31 (leg. Niketić, M. 29Aug-1997, det. G. Tomović, BEO, sub V. calcarata subsp. zoysii); Marijaš, Bogdaš, ispod vrha, UTM 
DN21 (leg. Džukić, G., Milojević, B. 16-Jul-1980, det. G. Tomović, 878 BEOU-KEGB, sub V. zoysii); Nedžinat, UTM DN22, osuline i kamenjari (leg. Nikolić, V., Diklić, N., Bogdanović, M. 15-Jul-1973, det. G. Tomović, 43185, 43186 BEO, sub $V$. zoysii); Žljeb (Maja Rusolija), UTM DN33, $1900 \mathrm{~m}$ (leg. Rudski, I. 25-Jun-1932, det. G. Tomović, BEO, sub V. zoysii); 2000 m (leg. Rudski, I. 25-Jun-1932, det. G. Tomović, VZ-001 BEO, sub V. zoysii); $2300 \mathrm{~m}$ (leg. Rudski, I. 25-Jun-1932, det. G. Tomović, VZ-002 BEO, sub V. zoysii); vrh, UTM DN33, $2100 \mathrm{~m}$, blizu vrha, pored snega (leg. Rudski, I. 25-Jun-1932, det. G. Tomović, VZ-004 BEO, sub V. zoysii).

Viola dacica Borbás (Fig. 20) NE Serbia Bor: Stol, UTM EP99 (leg. Pančić, J., Jun-1876, det. G. Tomović, 15002 BEOU (Herb. Panc.), sub Viola sp.); Đerdapska klisura: Donji Milanovac, UTM EQ82 (leg. Pančić, J. Jul-1871, det. J. Pančić, 3306 BEOU (Herb. Panc.), sub V. tricolor, rev. G. Tomović, 07-Sep-2013); Majdanpek, UTM EQ71, det. G. Tomović, 8420 BEO); (leg. Pančić, J. 1875, det. J. Pančić, 3189 BEOU (Herb. Panc.), sub V. speciosa, rev. G. Tomović, 23-Sep-2013); W Serbia Čemerno, UTM DP52, in sylvaticus (leg. Pančić, J., Aug1860, det. J. Pančić, 3258 BEOU (Herb. Panc.), sub V. nicolai, rev. G. Tomović, 07-Sep-2013); Zvezda: Podstolac, Štula karaula, Dugi do, UTM CP66, in pratis sylvestribus (leg. Pančić, J. Aug-1875, det. J. Pančić, 3261 BEOU (Herb. Panc.), sub V. nicolai var. serbica, rev. G. Tomović, 07-Sep-2013); (Jun-1912, 8430 BEO, sub $V$. polyodonta, rev. G. Tomović, Feb-2007; 8514, 8515 BEO, sub. V. saxatilis subsp. macedonica, rev. G. Tomović, Feb2007); (29-Aug-1887, 42039 BEOU, rev. G. Tomović, 07-Sep-2013); (det. G. Tomović, 8417 BEO); (leg. Soška, Th. 18-Jun-1912, det. Th. Soška, 42040 BEOU); C Serbia Ibarska

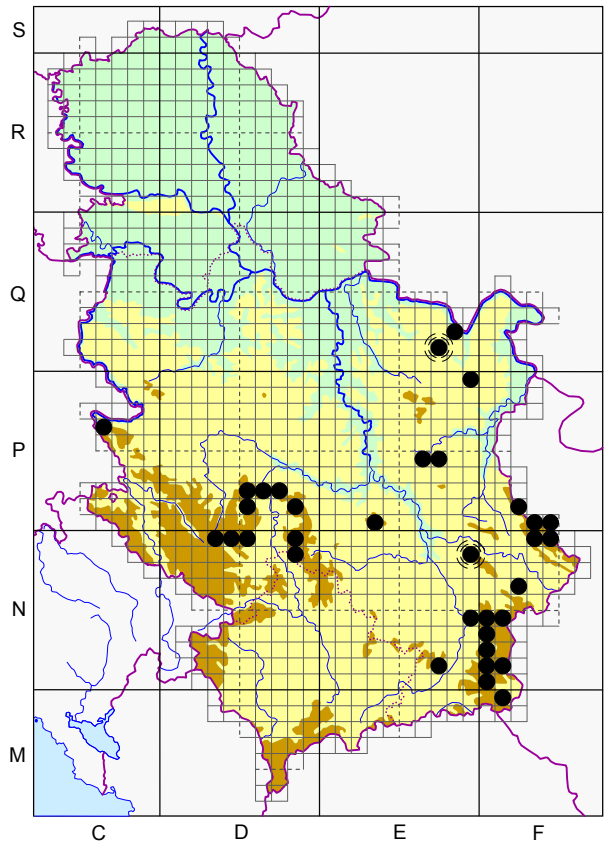

Fig. 20. - Distribution of the species $V$. dacica Borbás in Serbia.

dolina: Maglić grad, UTM DP62, serpentinit (leg. Niketić, M., Buzurović, U., 11-Jun-2014, det. G. Tomović, BEO); (40652 BEOU-KEGB); Jastrebac: Veliki Jastrebac, Kraljeva česma, UTM EP30 (leg. Diklić, N. 12-May-1959, det. N. Diklić, 42845 BEO, sub V. saxatilis subsp. macedonica, rev. G. Tomović, Feb-2007); Tri sestrice, vrh, UTM EP30, 1300 m (leg. Diklić, N. 25-Jul-1959, det. N. Diklić, 42844 BEO, sub V. saxatilis subsp. macedonica, rev. G. Tomović, Feb-2007); Kopaonik, granit (leg. Lakušić, D. 19-Apr-1986, det. D. Lakušić, 41249 BEOU, sub V. tricolor, rev. G. Tomović, Apr-2007); osuline pored puta (leg. Lakušić, D. 16-Sep-1986, det. D. Lakušić, 42025 BEOU); det. G. Tomović, 8415 BEO); (42010 BEOU); (leg. Lakušić, D. 07-Jun-1985, det. D. Lakušić, 42026 BEOU, sub V. tricolor subsp. tricolor, rev. G. Tomović, 07-Sep-2013); (leg. Pančić, J. 12-Jul-1886, det. J. Pančić, 15009 BEOU (Herb. Panc.), sub V. aetolica, rev. G. Tomović, Apr-2007); (leg. Pančić, J., det. J. Pančić, 3319 BEOU (Herb. Panc.), sub V. tricolor var. alpestris, rev. G. Tomović, Apr-2007); (leg. Rudski, I. 01-Jun-1938, det. I. Rudski, 8487 BEO, sub V. saxatilis subsp. macedonica, rev. G. Tomović, Feb-2007); Bećirovac, UTM DN89 (leg. Pančić, J. 1868, det. J. Pančić, 14600 BEOU (Herb. Panc.), sub V. macedonica (declinata), rev. G. Tomović, Apr-2007); Bela reka, UTM DN89, Exp. NE (leg. Mišić, V., Popović, M. 22-Jul-1952, det. G. Tomović, 42017 BEOU); Bele stene, UTM DN89 (leg. Rudski, I. Jul-1938, det. G. Tomović, 42848 BEO); (leg. Rudski, I. 12-Jul-1938, det. G. Tomović, 42849 BEO); Srebrnac, UTM DN89, livade (leg. Nikolić, V., Diklić, N. 14-Jul-1967, det. N. Diklić, 42838 BEO); vlažne livade u zoni Fagetum montanum (leg. Stevanović, V., 
Niketić, M. 04-Jun-1985, det. D. Lakušić, 42035 BEOU); Crni Jelak, UTM DN89, 1767 m, silikat, mezofilne livade (leg. Lakušić, D. 06-Jun-2007, det. G. Tomović, 24440 BEOU-KEGB); (leg. Lakušić, D. 06-Jun-2007, 24441 BEOU-KEGB, sub V. dacica × macedonica, rev. G. Tomović, 07-Sep-2013); Gobelja, Oštri krš, UTM DN89 (leg. Lakušić, D., Mitrović, V., "ENDEMIT" 26-May-2001, det. G. Tomović, 13897 BEOU-KEGB); Karaman, UTM DN89, 1900 m, granit, Vaccinio-Juniperetum nanae (leg. Lakušić, D. 15-Jul-1987, det. D. Lakušić, 42022 BEOU); Exp. NE, 1900 m, granodiorit, VaccinioJuniperetum nanae (leg. Lakušić, D. 15-Jul-1987, det. D. Lakušić, 42024 BEOU); 1800 m, silikat, pašnjaci, kleke (leg. Tomović, G. 29-May-2006, 21205 BEOU-KEGB, sub V. tricolor subsp. macedonica, rev. G. Tomović, 07-Sep-2013); (leg. Rudski, I. 09-Jul-1938, det. I. Rudski, 8480 BEO, sub V. saxatilis subsp. macedonica, rev. G. Tomović, Feb-2007); Mali Karaman, UTM DN89, 1800 m, granodiorit (leg. Lakušić, D. Jul-1987, det. D. Lakušić, 42019 BEOU); Metođe, UTM DN89, bukova šuma, 26-May-1952, det. G. Tomović, 42015 BEOU); Pajino preslo, UTM DN89, vlažne livade u smrčevoj šumi (leg. Nikolić, V. 17-Aug-1982, det. G. Tomović, 42837 BEO); Pančićev vrh, UTM DN89 (leg. Rudski, I. 08Jul-1938, det. Th. Soška, 8481 BEO, sub V. saxatilis subsp. macedonica, rev. G. Tomović, Feb-2007); (leg. Rudski, I. 13-Jul-1938, 8482 BEO, sub V. saxatilis subsp. macedonica, rev. G. Tomović, Feb-2007); Samokovska reka - klisura, UTM DN89, smrčeva šuma sa mahovinom (leg. Mišić, V., Popović, M. 09-Jun-1952, det. G. Tomović, 42016 BEOU); Suvi jelak, UTM DN89, 1700 m, granit, Piceetum excelsae (leg. Lakušić, D., Niketić, M. 16-Sep-1986, det. D. Lakušić, 42023 BEOU); Suvo rudište, UTM DN89, Exp. N, 1850 m, granit, Vaccinio-Juniperetum nanae (leg. Lakušić, D. 05-Jul-1991, det. D. Lakušić, 42021 BEOU); Exp. NW, 1800-1900 m, kornit (leg. Lakušić D., Niketić M. 07-Aug-1986, det. D. Lakušić, 42007 BEOU, sub V. tricolor subsp. subalpina, rev. G. Tomović, 07-Sep-2013); (leg. Rudski, I. 01-Jun-1938, det. G. Tomović, 42847 BEO); (leg. Rudski, I. Oct-1938, det. I. Rudski, 8543 BEO, sub V. saxatilis, rev. G. Tomović, Feb-2007); (leg. Rudski, I. 01-Jun-1938, det. P. Černjavski, 8486 BEO, sub $V$. saxatilis subsp. macedonica, rev. G. Tomović, Feb-2007); (leg. Rudski, I. Oct-1938, det. I. Rudski, 8467 BEO, sub V. elegantula var. latisepala, rev. G. Tomović, Feb-2007); (leg. Sigunov, A. 22.-23-Aug-1946, det. P. Černjavski, 8464 BEO, sub V. elegantula, rev. G. Tomović, Feb-2007); Treska, UTM DN88, serpentinit (leg. Niketić, M., Tomović, G., Novčić, R. 09-Jul-2005, det. G. Tomović, 19924 BEOU-KEGB); 1550 m, serpentinit, Fagetum montanum (leg. Lakušić, D. 05-Jul-1991, det. D. Lakušić, 42020 BEOU); Radočelo: vrh, UTM DP51 (leg. Soška, Th. 02-Jul-1932, det. Th. Soška, 42028 BEOU, sub $V$. polyodonta, rev. G. Tomović, 07-Sep-2013); Stolovi, UTM DP62, 8431 BEO, rev. G. Tomović, 07Sep-2013); (leg. Pančić, J. Jun-1864, det. J. Pančić, 3302 BEOU (Herb. Panc.), sub V. tricolor, rev. G. Tomović, 07-Sep-2013); (leg. Pančić, J. Jul-1877, det. J. Pančić, 3195 BEOU (Herb. Panc.), sub V. affinis prolixa Panč.); (leg. Pančić, J. 1866, det. J. Pančić, 15014 BEOU (Herb. Panc.), sub V. macedonica, rev. G. Tomović, 07-Sep-2013); (leg. Pančić, J. 1869, det. J. Pančić, 3305 BEOU (Herb. Panc.), sub V. tricolor; macedonica conf. dechinata, rev. G. Tomović, 07-Sep-2013); (leg. Pančić, J. 1877, det. J. Pančić, 15007 BEOU (Herb. Panc.), sub V. aff. prolixarani?, rev. G. Tomović, 07-Sep-2013); (leg. Soška, Th. 04-Jul-1924, det. Th. Soška, 42008 BEOU, sub V. saxatilis, rev. G. Tomović, Apr-2007); Studena planina: Cvetalica, UTM DP72, $1100 \mathrm{~m}$, serpentinit, livade pored puta (leg. Niketić, M., Buzurović, U. 28-Jun-2014, det. G. Tomović, BEO); (40777 BEOU-KEGB); Željin: Obrenovići, UTM DP81, 21-Jun-1989, det. G. Tomović, 42018 BEOU); E Serbia Babušnica: Stol, UTM FN26, 1930, det. G. Tomović, 8421 BEO); det. G. Tomović, 8413, 8422 BEO); Rtanj, UTM EP64, EP74 (leg. Pančić, J., det. G. Tomović, 3177 BEOU (Herb. Panc.), sub Viola sp.); (leg. Soška, Th. Jun-1924, det. G. Tomović, 41181, 3262 BEOU, sub V. alpestris, rev. G. Tomović, 07-Sep-2013); (leg. Stevanović, V. 15Jun-1974, det. G. Tomović, 10218, 10440 BEOU-KEGB); Stara planina, 04-Jun-1911, det. G. Tomović, 42027 BEOU); det. G. Tomović, 42037 BEOU); Arbinje, UTM FN49 (leg. Tomović, G., Zlatković, B. 27-Jul-1998, 11646 BEOU-KEGB, sub V. tricolor, rev. G. Tomović, 07-Sep-2013); Babin zub, UTM FP30, 1500 m (leg. Ranđelović, V., Zlatković, B., Vukojičić 08-Jun-1997, det. G. Tomović, 5714, 5739 BEOU-KEGB); 1700 m (leg. Grebenščikov, O., Rajković, J. 11-May-1948, det. G. Tomović, 42012 BEOU); 1700 m, pašnjaci i kamenjari (leg. Nikolić, V., Diklić, N. 27-Jun-1975, det. G. Tomović, 42836 BEO); (leg. Pančić, J. 1880, det. J. Pančić, 3313 BEOU (Herb. Panc.), sub V. tricolor, rev. G. Tomović, 07-Sep-2013); (leg. Tomović, G. 08-Jun-1997, det. G. Tomović, 5709 BEOU-KEGB); Dojkinačka reka - izvorište, UTM FN49, 1550 m, crveni peščar i konglomerat, bukova šuma (leg. Mihailović, T. 27-Jul1993, det. T. Mihailović, 42030 BEOU, sub V. tricolor, rev. G. Tomović, 2005); Dupljak, UTM FP30, 
granit, visokoplaninske livade (leg. Mihailović, T. 25-Jul-1993, det. T. Mihailović, 42029 BEOU, sub V. tricolor, rev. G. Tomović, 2005); visokoplaninske livade (leg. Mihailović, T. 25-Jul-1993, det. T. Mihailović, 42033 BEOU); Gornji Rišor, UTM FN49, FP40, 1300 m (leg. Grebenščikov, O., Rajković, J. 13May-1948, det. O. Grebenščikov, 42011 BEOU); Midžor, UTM FP30, crveni peščar i konglomerat, visokoplaninske pašnjaci (leg. Mihailović, T. 29-Jul-1993, det. T. Mihailović, 42034 BEOU, sub V. tricolor subsp. subalpina, rev. G. Tomović, 2005); (leg. Pančić, J. 1879, det. G. Tomović, 3198 BEOU (Herb. Panc.), sub Viola sp.); (leg. Pančić, J., det. J. Pančić, 8416 BEO); Dugo bilo, UTM FP30, 1600 m, livade (leg. Nikolić, V. 23-Jun-1958, det. V. Nikolić, 42840 BEO, sub V. saxatilis subsp. macedonica, rev. G. Tomović, Feb-2007); (leg. Nikolić, V. 23-Jun-1958, det. V. Nikolić, 42841, 42842 BEO, sub V. saxatilis subsp. macedonica, rev. G. Tomović, 23-Sep-2013); Sveti Nikola, UTM FP21, det. G. Tomović, 8414 BEO); (leg. Pančić, J. 1878, det. J. Pančić, 3323 BEOU (Herb. Panc.), sub V. tricolor var. balcanica, rev. G. Tomović, 07-Sep-2013); Topli do, UTM FN49, 1700 m (leg. Grebenščikov, O. 04-Jul-1946, det. O. Grebenščikov, 8497 BEO, sub $V$. saxatilis subsp. macedonica, rev. G. Tomović, Feb-2007); BelanVrtibog, UTM FN39, FN49, 1200-1900 m (leg. Ostojić, D. 15-Jun-1998, det. G. Tomović, 11435 BEOUKEGB); Bratkova strana, UTM FN49, 1700 m (leg. Grebenščikov, O. 04-Jul-1946, det. O. Grebenščikov, 8499 BEO, sub V. saxatilis subsp. macedonica, rev. G. Tomović, Feb-2007); Krvave bare, UTM FN49, Juniperetum (leg. Niketić, M. 15-Sep-2013, det. G. Tomović, BEO); (leg. Ružić, A., Petrov 15-May1947, det. O. Grebenščikov, 8412 BEO); Tri čuke, UTM FN49 (leg. Pančić, J. 1881, det. J. Pančić, 15005 BEOU (Herb. Panc.), sub V. tricolor, rev. G. Tomović, 07-Sep-2013); Tri čuke-Krvave bare, UTM FN49 (leg. Pančić, J. 1882, det. J. Pančić, 15004 BEOU (Herb. Panc.), sub V. tricolor var., rev. G. Tomović, 07-Sep-2013); Tupanar, UTM FP30, crveni peščar i konglomerat (leg. Mihailović, T. 24-Jul1993, det. T. Mihailović, 42032 BEOU); Udica, UTM FP30 (leg. Nikolić, V., Diklić, N. 23-Jun-1958, det. V. Nikolić, 42843 BEO, sub $V$. saxatilis subsp. macedonica, rev. G. Tomović, Feb-2007); Žarkova čuka, UTM FP30, crveni peščar i konglomerat, planinski pašnjaci (leg. Mihailović, T. 24-Jul-1993, det. T. Mihailović, 42031 BEOU, sub V. tricolor, rev. G. Tomović, 2005); UTM FP30, 1650 m, silikat (leg. Niketić, M., Tomović, G. 31-Aug-2002, det. G. Tomović, 15867 BEOU-KEGB); 1690 m, silikat (leg. Niketić, M., Tomović, G. 31-Aug-2002, det. G. Tomović, BEO); $1850 \mathrm{~m}$, silikat, stene i rudine (leg. Stevanović, V., Niketić, M., Tomović, G. 17-Jul-1998, det. G. Tomović, 11536 BEOU-KEGB); Exp. N, 1900 m, silikat, stene (leg. Stevanović, V., Niketić, M., Tomović, G. 17-Jul-1998, det. G. Tomović, 11574 BEOU-KEGB); Suva planina, UTM EN98, in rupestribus (leg. Pančić, J. 1883, det. G. Tomović, 14994 BEOU (Herb. Panc.), sub Viola sp.); SW Serbia Golija (leg. Pančić, J., det. J. Pančić, 3228 BEOU (Herb. Panc.), sub V. declinata (lutea); V. macedonica, rev. G. Tomović, 07-Sep-2013); Crni vrh - Šeremetovica, UTM DN59 (leg. Grebenščikov, O., Mišić, V., Janković, M. 09-Aug-1949, det. G. Tomović, 42038 BEOU, sub V. macedonica, rev. G. Tomović, 24-Apr-2013); Jankov kamen, UTM DN49, Exp. SW, 1799 m, granodiorit (kvarclatit) (leg. Đorđević, v. 10-Aug-2014, det. G. Tomović, 40645 BEOU-KEGB); $1770 \mathrm{~m}$, silikat (leg. Đorđević, V. 22-Jun-2014, det. G. Tomović, 40644 BEOU-KEGB); Preko brdo, UTM DN39 (leg. Vukojičić, S. 22-Aug-2002, det. G. Tomović, 16206 BEOU-KEGB); S Serbia Pljačkavica, UTM EN71 (leg. llić, Đ., det. Đ. Ilić, 8418 BEO); SE Serbia Besna kobila: ispod vrha, UTM FN00 (leg. Nikolić, V., Diklić, N., 22-Jul-1967, det. N. Diklić, 43087 BEO, sub V. saxatilis subsp. macedonica, rev. G. Tomović, 23-Sep-2013); Dukat planina: Crnook, Bistar-Jarešnik, UTM FM19, silikat (leg. Niketić, M. 10-Oct-2010, det. G. Tomović, BEO); Milevska planina: vrh Bandera, UTM FN11, 1736 m, silikati (leg. Niketić, M. 14-Sep-2006, det. G. Tomović, 2006913 BEO); (40651 BEOUKEGB); Ostrozub: Polom, UTM EN94 (leg. Niketić, M., Tomović, G. 30-Apr-2008, 26850 BEOUKEGB); Vardenik: Strešer, UTM FN01, FN02 (leg. Dimitrijević, M. J. 26-Jun-1911, det. Ž. J. Jurišić, 8491 BEO, sub V. macedonica, rev. G. Tomović, Feb-2007); Veliki Strešer, UTM FN01, FN02, 1500-1600 m (leg. Nikolić, V., Diklić, N. 23-Jul-1967, det. N. Diklić, 42846 BEO, sub V. saxatilis subsp. macedonica, rev. G. Tomović, Feb-2007); Vlasina (leg. Pančić, J., det. J. Pančić, 3299 BEOU (Herb. Panc.), sub V. tricolor, rev. G. Tomović, 07-Sep-2013); Plana, vrh, UTM FN04, FN14, kamenjari (leg. Černjavski, P. 16-Aug-1930, det. P. Černjavski, 8521 BEO, sub V. saxatilis subsp. macedonica, rev. G. Tomović, Feb-2007); vodojaža, iznad, UTM FN03, pored potoka (leg. Černjavski, P., Pavlović, J., Mišić, v. 24-May-1947, det. G. Tomović, 42013 BEOU). 
Viola dukadjinica W. Becker \& Košanin (Fig. 21)

Kosovo Šar-planina: Kodža Balkan, Ostrovica, UTM DM97, $1910 \mathrm{~m}$, serpentinit, stene i kamenjari (leg. Lazarević, M., Lazarević, P., 17-Aug-2011, det. G. Tomović, 33165 BEOUKEGB); (leg. Stevanović, V. 26-Jun-1997, det. V. Stevanović, 7245 BEOU-KEGB, rev. G. Tomović, 07-Sep-2013); (leg. Stevanović, V., Jovanović, S. 20-Jul-1989, 200 BEOU-KEGB).

Viola elegantula Schott (Fig. 22) W Serbia Čemernica, UTM DP11 (leg. Pančić, J., 1866, det. J. Pančić, 15001 BEOU (Herb. Panc.), sub V. lutea; V. calcarata var. flava); SW Serbia Pešter: Karajukića bunari, skretanje za Suvi do, UTM DN26, krečnjak, livade pored puta (leg. Niketić, M., Tomović, G., Buzurović, U., 11-May-2014, det. G. Tomović, 40637 BEOU-KEGB); Karajukića bunari - Boroštica, UTM DN26, $1173 \mathrm{~m}$ (leg. Tomović, G., Jakovljević, K., Đurović, S., Buzurović, U. 281-May-2014, det. G. Tomović, 40666 BEOU-KEGB); Peštersko polje, Suvi do, ispred, UTM DN26, livade (leg. Košanin, N. 22-Jun-1926, det. G. Tomović, 41230 BEOU, sub $V$. macedonica, rev. G. Tomović, 07-Sep2013); Prijepolje: Kamena Gora, Gluščevići, UTM CN89, Exp. N, 1216 m, krečnjak, Brometum erecti (leg. Đorđević, V. 18-Jul-2014, det. G. Tomović, 40648 BEOU-KEGB); Gluščevići (česma Vučetina), UTM CN89, Exp. SE, 1210 m, krečnjak, Brometum erecti (leg. Đorđević, V. 18-Jul-2014, det. G. Tomović, 40649 BEOU-KEGB); Stari Kolašin: Batrage, Draška suhovara, UTM DN55, bukovo-smrčeva šuma (leg. Rudski, I. 06-Jul-1932, det. G. Tomović, 42892 BEO); Kosovo Šar-planina (leg. Stevanović, V., Jovanović, S., 21-Jul1989, det. G. Tomović, 3306, 3355 BEOUKEGB); Brezovica, Borovik, UTM DM97, serpentinit (leg. Nikolić, V., Diklić, N., Mladenović, S. 29-Jul-1972, det. G. Tomović, BEO, sub $V$. latisepala); (leg. Nikolić, V., Diklić, N., Mladenović, S. 29-Jul-1972, det. G. Tomović, BEO, sub $V$. latisepala); Gradište, UTM EM07, $900 \mathrm{~m}$, serpentinit (leg. Stevanović, V. 02-Sep-1997, det. V. Stevanović, 7729 BEOU-KEGB, sub $V$. dukadjinica, rev. G. Tomović, 07-Sep-2013); Lepenac reka, leva obala, UTM DM97, EM07, serpentinit (leg. Jovanović, S., Lakušić, D., Benić, N. 30-Jun-1990, det. G. Tomović, 154190 BEOU-KEGB, sub $V$. macedonica); Lepenac reka, pored, UTM DM97, EM07, serpentinit (leg. Nikolić, V., Diklić, N., Mladenović, S. 20-

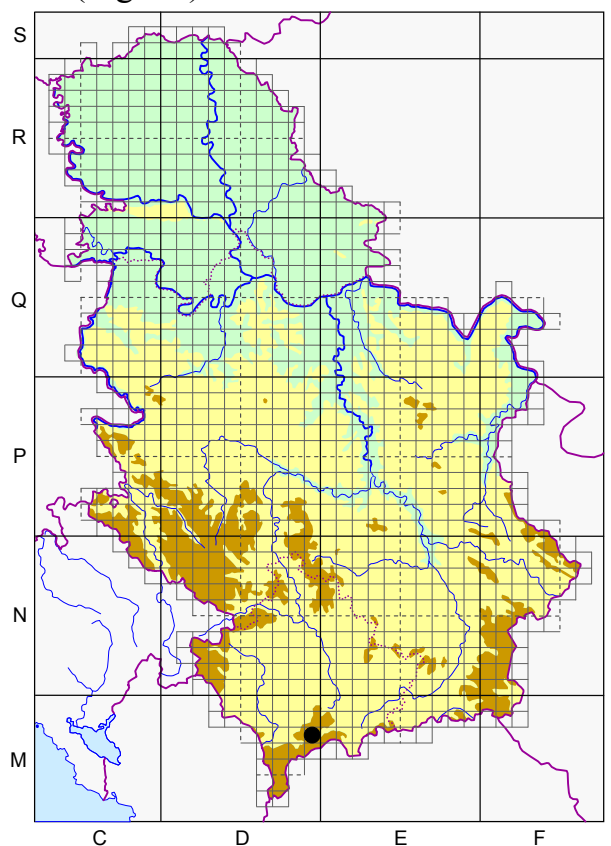

Fig. 21. - Distribution of the species V. dukadjinica W. Becker \& Košanin.

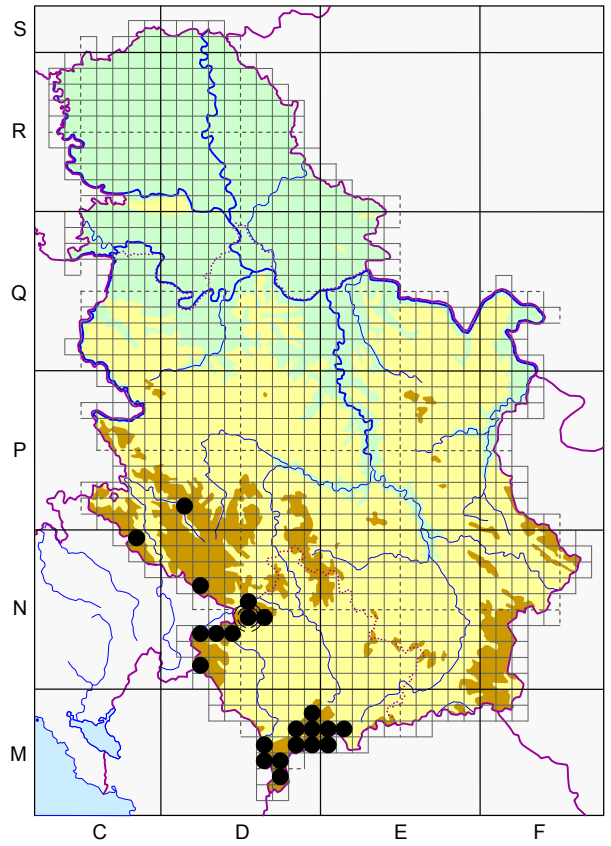

Fig. 22. - Distribution of the species $V$. elegantula Schott in Serbia. 
Jul-1974, det. G. Tomović, BEO, sub V. latisepala); (leg. Nikolić, V., Diklić, N., Mladenović, S. 26-Jul-1976, det. G. Tomović, BEO, sub $V$. latisepala); Brezovica-Stojkova kuća-Piribeg, UTM EM06, EM07, kamenjari (leg. Nikolić, V., Diklić, N., Bogdanović, M. 22-Jul-1974, det. G. Tomović, 42854 BEO); Careve livade, UTM DM96, 1600 m, Juniperetum, zona Pinus peuce, Pinus heldreichii, 41282 BEOU, rev. G. Tomović, Apr-2007); munikovo-beloborova šuma (leg. Janković, M. 08-Jul-1961, det. M. Janković, 41264 BEOU, sub $V$. saxatilis, rev. G. Tomović, Apr-2007); Careve livade-Jažinačko jezero, UTM DM96, EM06, 1200-2100 m (leg. Nikolić, V., Diklić, N., Mladenović, S. 24-Jul-1980, det. G. Tomović, 42859, 42860 BEO); Durlov potok, UTM EM06 (leg. Sabovljević, M. 29-Jun-1997, det. G. Tomović, 7427 BEOU-KEGB); Gine vode, UTM DM96, munikova šuma (leg. Janković, M. 08-Jun-1965, det. G. Tomović, 41263 BEOU); Gine vode-Mekuš bor-Careve livade, UTM DM96, $1900 \mathrm{~m}$ (leg. Stevanović, V. 24-Jun-1997, det. G. Tomović, 7343 BEOU-KEGB); Gine vode-Prevalac-Štrpce, UTM DM96, EM07 (leg. Janković, M. 08-11-Jun-1965, det. G. Tomović, 41262 BEOU); Kodža Balkan, UTM DM87, DM97, 1600-2090 m, serpentinit, pašnjaci i kamenjari (leg. Nikolić, V., Diklić, N. 18-Jul-1975, det. N. Diklić, 42876 BEO, sub V. saxatilis subsp. macedonica, rev. G. Tomović, Feb2007); Exp. SE, 1600-2090 m, serpentinit, pašnjaci i kamenjari (leg. Nikolić, V., Diklić, N. 18-Jul-1975, det. G. Tomović, BEO, sub $V$. latisepala); Cerenački vrh, UTM DM87, DM97, 1500-2040 m, krečnjak (leg. Nikolić, V., Diklić, N. 19-Jul-1975, det. G. Tomović, 42872 BEO); Maja Studenica, UTM DM98, livade (leg. Nikolić, V. 22-Jun-1962, det. G. Tomović, BEO, sub V. latisepala); Malo Borče, UTM DM97, 1400-1512 m, serpentinit, kamenjari (leg. Nikolić, V., Diklić, N., Mladenović, S. 21-Jul-1974, det. G. Tomović, BEO, sub V. latisepala); serpentinit, kamenjari (leg. Nikolić, V., Diklić, N., Mladenović, S. 27-Jul1976, det. G. Tomović, BEO, sub V. latisepala); Ostrovica, UTM DM97, 1910 m, serpentinit, stene i kamenjari (leg. Lazarević, M., Lazarević, P. 17-Aug-2011, 33166 BEOU-KEGB, sub V. serbica sp. nova, rev. G. Tomović, 07-Sep-2013); (leg. Stevanović, V. 26-Jun-1997, det. V. Stevanović, 7240 BEOUKEGB, rev. G. Tomović, 07-Sep-2013); (leg. Stevanović, V., Jovanović, S. 20-Jul-1989, 279-89, 232-89 BEOU-KEGB, rev. G. Tomović, 07-Sep-2013); Ostrovica, iznad Sevce, UTM DM97, serpentinit, kamenjari (leg. Zlatković, B. Jul-1995, det. B. Zlatković, 29040 BEOU-KEGB, sub V. nova, rev. G. Tomović, 07-Sep-2013); Ostrovica, Zavoj, UTM DM97, 1700 m, serpentinit, Pinetum heldreichii (leg. Niketić, M., Tomović, G., Duraki Š. 10-Jul-2007, 24535 BEOU-KEGB, sub V. nova, rev. G. Tomović, 07Sep-2013); Livadica, UTM EM07, livade i rudine (leg. Stevanović, V. 05-Jul-1977, det. G. Tomović, 3464 BEOU-KEGB); (leg. Košanin, N. 06-Jul-1911, det. N. Košanin, 41273 BEOU, sub V. gracilis, rev. G. Tomović, Apr-2007); (leg. Stevanović, V. 05-Jul-1977, det. G. Tomović, 3466 BEOU-KEGB); Livadica-Piribeg, UTM EM06, EM07, 2300 m, rudine, pašnjaci i stene (leg. Stevanović, V. 07-Jul1980, det. G. Tomović, 1988 BEOU-KEGB); Ljuboten, Bačije, UTM EM17 (leg. Bierbach, O. Jun1900, det. G. Tomović, 41266 BEOU, sub V. latisepala); Bačije, iznad, UTM EM17 (leg. Soška, Th. 04Jul-1914, det. Th. Soška, 41272 BEOU, sub V. alpestris subsp. aetolica, rev. G. Tomović, Apr-2007); (leg. Soška, Th. Jul-1914, det. Th. Soška, 41268 BEOU, sub V. latisepala); Bačije-prevoj, UTM EM07, EM17, silikat (leg. Soška, Th. 05-Jul-1914, det. Th. Soška, 41269 BEOU, sub V. latisepala); (leg. Soška, Th. 05-Jul-1914, det. Th. Soška, 41270 BEOU, sub V. latisepala); ispod vrha, UTM EM07 (leg. Košanin, N. 06-Jul-1911, det. N. Košanin, 41267 BEOU, sub V. latisepala); Jezero, UTM EM17, Jun-1910, det. G. Tomović, 41265 BEOU); Mandra, UTM EM07 (leg. Bierbach, O. Jun-1900, det. O. Bierbach, 41271 BEOU, sub V. alpestris subsp. aetolica, rev. G. Tomović, Apr-2007); podnožje, UTM EM07, 1550 m, krečnjak+silikat, Vaccinio-Juniperetum nanae (leg. Stevanović, V. 01-Sep-1997, det. G. Tomović, 7856 BEOU-KEGB); Ošljak, UTM DM87, DM97 (leg. Lindtner, v. Oct-1937, det. G. Tomović, BEO); Berova glava, UTM DM97 (leg. Rudski, I. 22-Jul-1930, det. I. Rudski, 41280 BEOU); Bivolje, UTM DM97, in pratis alpinis (leg. Soška, Th. 07-Jun-1923, det. Th. Soška, 41288 BEOU, sub V. gracilis, rev. G. Tomović, 07-Sep-2013); (leg. Jakovljević, S. 07-Jun-1923, det. S. Jakovljević, 41287 BEOU, sub V. latisepala); (leg. Soška, Th. 07-Jun-1925, det. Th. Soška, 41286 BEOU, sub V. gracilis, rev. G. Tomović, 07-Sep-2013); Golem Bor, UTM DM87, 1700 m, krečnjak+silikat (leg. Stevanović, v. 04-Sep-1997, det. G. Tomović, 7869 BEOU-KEGB); Markova glava-Jezera, UTM DM97 (leg. Košanin, N. 30-May-1922, det. W. Becker, 41283 BEOU, sub V. alpestris subsp. aetolica, rev. G. Tomović, Apr-2007); Popovo prase, UTM DM97, livade (leg. Nikolić, V., Diklić, N., Mladenović, S. 23-Jul-1976, det. G. Tomović, 42858 BEO); Popovo prase-Ostrovica, UTM DM97, 750-1800 m, 23-26-Aug-1966, det. G. Tomović, 41254 BEOU); Prevalac, UTM DM96, 1600 m, livade (leg. Nikolić, V., Diklić, N. 13-Jul-1968, det. N. Diklić, 43090, 43091 BEO, sub V. saxatilis subsp. macedonica, rev. G. Tomović, 23-Sep-2013); Ravna brezaJezera, UTM DM97 (leg. Košanin, N. 30-May-1922, det. N. Košanin, 41285 BEOU); (leg. Košanin, N. 30- 
May-1922, det. N. Košanin, 41284 BEOU, sub V. saxatilis subsp. aetolica, rev. G. Tomović, Apr-2007); Virovi, UTM DM87, DM97, 1800 m (leg. Rudski, I. 22-Jul-1930, det. I. Rudski, 8447 BEO, sub V. elegantula var. latisepala); (leg. Rudski, I. 12-Jul-1930, det. I. Rudski, 41278 BEOU); (leg. Rudski, I. 22Jul-1930, det. G. Tomović, 41279, 41281 BEOU); Piribeg, UTM EM06 (leg. Stevanović, V., Jovanović, S. 21-Jul-1989, det. G. Tomović, 3294 BEOU-KEGB); Stojkova kuća, UTM EM06, $1800 \mathrm{~m}$ (leg. Stevanović, V. 07-Jul-1980, det. G. Tomović, 3078 BEOU-KEGB); silikat, Vaccinio-Juniperetum-Rhododendron ferrugineum (leg. Stevanović, V. 26-Jun-1997, det. G. Tomović, 7301, 7306, 7308 BEOUKEGB); Stojkova kuća-Durlov potok, UTM EM06, 1600-1900 m, livade i kamenjari (leg. Nikolić, V., Diklić, N., Mladenović, S. 25-Jul-1976, det. G. Tomović, 42861, 42862, 42863 BEO); (leg. Nikolić, V., Diklić, N., Mladenović, S. 25-Jul-1976, det. N. Diklić, 42871 BEO); Stojkova kuća-Jezerska čuka, UTM EM06, 1600-2400 m (leg. Nikolić, V., Diklić, N., Mladenović, S. 23-Jul-1980, det. G. Tomović, 42855, 42856, 42857 BEO); Stojkova kuća-Livadica, UTM EM07 (leg. Džukić, G. 02-Jul-1980, det. G. Tomović, 3416 BEOU-KEGB); Prevalac, gornje sedlo, UTM DM96 (leg. Košanin, N. 29-May-1922, det. N. Košanin, 41257 BEOU); Metohija Koritnik, UTM DM65, DM66, in pascuis (leg. Oehm, H., 15-Jun-1936, det. O. Grebenščikov, 8479 BEO, sub V. elegantula var. latisepala); (leg. Oehm, H. 15-Jun-1936, det. Th. Soška, O. Grebenščikov, 8468 BEO, sub V. elegantula var. latisepala); Mokra gora, UTM DN54, vlažne livade (leg. Rudski, I. 16-Jul-1932, det. G. Tomović, 42887 BEO); (leg. Rudski, I. 16-Jul-1932, det. G. Tomović, 42888 BEO); Džora, UTM DN43, 1800 m (leg. Rudski, I. 28-Jun-1932, det. G. Tomović, 42890 BEO); (leg. Rudski, I. 27-Jun-1932, det. G. Tomović, 42882, 42889 BEO); Mokra planina, Jarebinje, UTM DN64, smrčeva šuma (leg. Rudski, I. 16-Jul-1932, det. G. Tomović, 42877 BEO); Radopolje, UTM DN64, 1750 m (leg. Rudski, I. 17-Jul-1932, det. G. Tomović, 42894 BEO); Prizren: Prizrenska Bistrica - klisura, UTM DM87 (leg. Stevanović, V. 09-Jul-1977, det. G. Tomović, 2915 BEOU-KEGB); Prokletije: Derviš Kom, UTM DN21 (leg. Rudski, I. Jul-1933, det. G. Tomović, 42893 BEO); Hajla, UTM DN23, DN33, 1900 m (leg. Gošović, S. Aug-1922, det. S. Gošović, 41290 BEOU); Krstac-Hajla, UTM DN23 (leg. Gošović, S. Aug-1922, det. S. Gošović, 41289 BEOU, sub $V$. elegantula subsp. latisepala); Peć-Rusolovac (leg. Janković, M. 21-Jun-1957, det. G. Tomović, 41291 BEOU); Žljeb (Maja Rusolija), UTM DN33, 1400 m (leg. Rudski, I. 25-Jun-1932, det. G. Tomović, 42880 BEO); 1500 m (leg. Rudski, I. 25-Jun-1932, det. G. Tomović, 42886 BEO); 1510 m (leg. Rudski, I. 25-Jun-1932, det. G. Tomović, 42885 BEO); 1900 m (leg. Rudski, I. 25-Jun-1932, det. G. Tomović, 42883 BEO); 1600-1700 m (leg. Rudski, I. 25-Jun-1932, det. G. Tomović, 42884 BEO); Alagin vrh, UTM DN33, $1800 \mathrm{~m}$ (leg. Rudski, I. 27-Jun-1932, det. G. Tomović, 42881 BEO); Kulina, ispod, UTM DN33 (leg. Košanin, N. 22-Jun-1922, det. N. Košanin, 41315 BEOU, sub V. latisepala); Žljeb (Maja Rusolija)-Peć, UTM DN33 (leg. Janković, M. 21-Jun-1957, det. G. Tomović, 41292 BEOU); Šarplanina: Bistrica, UTM DM96, in pratis subalpinis (leg. Soška, Th. 20-Jun-1924, det. Th. Soška, 41277 BEOU, sub V. latisepala); Ploča, UTM DM96, in pascuis alpinis (leg. Soška, Th. 20-Jun-1924, det. Th. Soška, 41274 BEOU, sub V. latisepala); (leg. Soška, Th. 16-Jun-1924, det. Th. Soška, 41275 BEOU, sub V. gracilis, rev. G. Tomović, Apr-2007); (leg. Soška, Th. 20-Jun-1924, det. Th. Soška, 41276 BEOU, sub $V$. gracilis, rev. G. Tomović, Apr-2007); Brod-Dragaš, UTM DM74, DM75, Exp. SW (leg. Nikolić, V., Diklić, N. 11-Jul-1968, det. N. Diklić, 42874 BEO, sub V. gracilis, rev. G. Tomović, Feb2007); Crni vrh, UTM DM96, 2200 m (leg. Rudski, I. 05-Jul-1930, det. I. Rudski, 41259 BEOU, sub V. latisepala); (leg. Rudski, I. Jul-1930, det. I. Rudski, 8448 BEO, sub V. elegantula var. latisepala); vrh, UTM DM96, 1850 m (leg. Rudski, I. 05-Jul-1930, det. G. Tomović, 41260 BEOU); Drajčići-Crni vrh, UTM DM96, 2000-2600 m, kamenjari (leg. Nikolić, V., Diklić, N. 17-Jul-1977, det. G. Tomović, 42867, 42868, 42869, 42870 BEO); livade, pašnjaci i kamenjari (leg. Nikolić, V., Diklić, N., Mladenović, S. 18-Jul1978, det. G. Tomović, 42865, 42866 BEO); Globočica, UTM DM65, 14-Jun-2010, 31057 BEOUKEGB, rev. G. Tomović, 07-Sep-2013); Kobilica, UTM DM86, DM96, 2400 m, pašnjaci i kamenjari (leg. Nikolić, V., Diklić, N., Mladenović, S. 19-Jul-1977, det. G. Tomović, 42864 BEO); (leg. Rudski, I. 1939, det. P. Černjavski, 8377 BEO, sub V. gracilis, rev. G. Tomović, Feb-2007); Karaula-Ljubinske uši, UTM DM86, 1900-2300 m (leg. Niketić, M., Tomović, G., Duraki, Š. 08-Jul-2007, det. G. Tomović, 24512 BEOU-KEGB, sub V. latisepala); Konjuška, Mlake, UTM DM96, 1600 m (leg. Rudski, I. 11-Jul-1930, det. I. Rudski, 41258 BEOU, sub $V$. latisepala); Ošljak, Popovo prase, UTM DM97, Exp. N, krečnjak, erodirana mesta u zoni Pinus heldreichii (leg. Niketić, M., Duraki, Š. 30-Jul-2008, det. G. Tomović, 29041 BEOU-KEGB, sub V. latisepala); Prevalac-Bistrica, UTM DM96, 1600-2200 m, krečnjak, kamenjari (leg. Nikolić, V., Diklić, N. 23-Jul-1975, det. N. Diklić, 42875 BEO, sub V. saxatilis subsp. macedonica, rev. G. Tomović, Feb-2007); Rudoka, Džinibeg, UTM DM74, DM75, pašnjaci (leg. 
Košanin, N. 14-Jul-1911, det. N. Košanin, 41256 BEOU); (leg. Košanin, N. 14-Jul-1911, det. N. Košanin, 41255 BEOU, sub V. gracilis, rev. G. Tomović, Apr-2007).

Viola grisebachiana Vis. (Fig. 23)

NE Serbia Bor: Veliki krš, greben, UTM EP89, 1000-1150 m (leg. Nikolić, V., Diklić, N., Bogdanović, M., 17-Jun-1972, det. G. Tomović, 42906, 42907, 42908 BEO); E Serbia Rtanj, UTM EP64, EP74 (leg. Lindtner, V. 11-Jun-1939, det. V. Lindtner, 8275, 8280, 8281 BEO); (leg. Pančić, J. Jul-1847, det. J. Pančić, 15052 BEOU (Herb. Panc.), sub V. grisebachiana (calcarata); prov. var. alpina); (leg. Pančić, J. 1869, det. J. Pančić, 14997 BEOU (Herb. Panc.)); (leg. Pančić, J. 1872, det. J. Pančić, 14995 BEOU (Herb. Panc.)); (leg. Pančić, J. 1874, det. J. Pančić, 14996 BEOU (Herb. Panc.)); greben, UTM EP64, EP74, 1300 m, stene (leg. Diklić, N. 09-Jun-1965, det. N. Diklić, 42903 BEO); kamenjari (leg. Nikolić, V., Diklić, N. 29-Jun-1971, det. N. Diklić, 42900, 42901, 42902 BEO); (leg. Nikolić, V., Diklić, N., Bogdanović, M. 20-Jun-1972, det. G. Tomović, 42909 BEO); Suva planina (leg. Petrović, S., May-1883, det. S. Petrović, 41435 BEOU); (leg. Petrović, S. 1912, det. S. Petrović, 41436 BEOU, sub V. grisebachiana radoslavivii); (leg. Petrović, S., det. G. Tomović, 41437 BEOU, sub $V$. grisebachiana radoslavivii); Devojački grob-Trem, UTM EN98, krečnjak (leg. Vukojičić, S., Tomović, G. 08-Jul1997, det. G. Tomović, 6291 BEOU-KEGB);

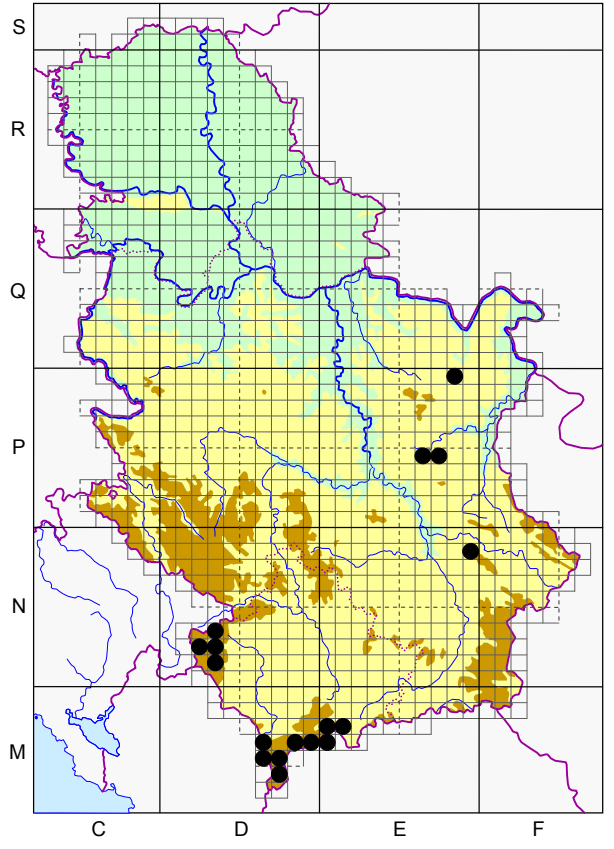

Fig. 23. - Distribution of the species $V$. grisebachiana Vis. in Serbia. UTM EN98, 1200-1700 m, krečnjak, pašnjaci i kamenjari (leg. Diklić, N. 31-May-1985, det. G. Tomović, 42911 BEO); Kosovo Šar-planina: Livadica-Piribeg, UTM EM06, EM07, 2250 m, krečnjak, stene i rudine (leg. Stevanović, V., 02-Jul-1980, det. G. Tomović, 41438 BEOU); Ljuboten, UTM EM07, EM17, Exp. E, 2300 m, in detritus calcareis (leg. Niketić, M., Stevanović, V. 12-Aug-1987, det. M. Niketić, VG-003 BEO); (leg. Bierbach, O. 15-Jul-1900, det. O. Bierbach, 41440 BEOU); vrh, UTM EM07 (leg. Bierbach, K. Jun-1900, det. K. Bierbach, 41441 BEOU); Piribeg, cirk, UTM EM06, $2200 \mathrm{~m}$ (leg. Stevanović, V. 19-Jul-1989, det. G. Tomović, 3489 BEOU-KEGB); Crvene karpe, UTM EM06 (leg. Stevanović, v. 04-Sep-1997, det. G. Tomović, 7976 BEOU-KEGB); Metohija Koritnik, UTM DM65, DM66 (leg. Rudski, I., 03-Aug-1936, det. G. Tomović, VG-002 BEO, sub V. grisebachiana Vis.); Prokletije: Koprivnik, UTM DN31, DN32, 2400 m, in glareosis (leg. Grebenščikov, O. 07-Jul-1939, det. G. Tomović, VG-003 BEO, sub V. grisebachiana Vis.); Lumbardska planina, UTM DN22, DN32, munikova šuma (leg. Janković, M. 23-May-1959, det. G. Tomović, 41434 BEOU); Streočka planina, UTM DN31, Exp. SE, munikova šuma (leg. Janković, M. 05-Jul- 1959, det. M. Niketić, 41433 BEOU); Žljeb (Maja Rusolija), vrh, UTM DN33, 2270 m, krečnjak, in rupestribus (leg. Černjavski, P., Rudski, I., Lindtner, V. 02-Aug-1932, det. P. Černjavski, VG-001 BEO, sub V. grisebachiana Vis.); Šarplanina: Kobilica, UTM DM86, DM96, 1800-2300 m, pašnjaci i kamenjari (leg. Nikolić, V., Diklić, N., Mladenović, S. 18-Jul-1978, det. G. Tomović, 42910 BEO); Rudoka, Džinibeg, UTM DM74, DM75, 2000-2310 m (leg. Stevanović, V., det. G. Tomović, 41439 BEOU).

Viola hymettia Boiss. \& Heldr. (Fig. 24)

NE Serbia Bor: Brestovačka banja, Kljancu Mare, UTM EP87 (leg. Pančić, J., 1867, det. J. Pančić, 3303 BEOU (Herb. Panc.), sub V. tricolor ?); E Serbia Niš: Donja Toponica, Popova glava, UTM EP60, silikat (leg. Niketić, M., Tomović, G., 03-Apr-2010, BEO, rev. G. Tomović, 23-Sep- 


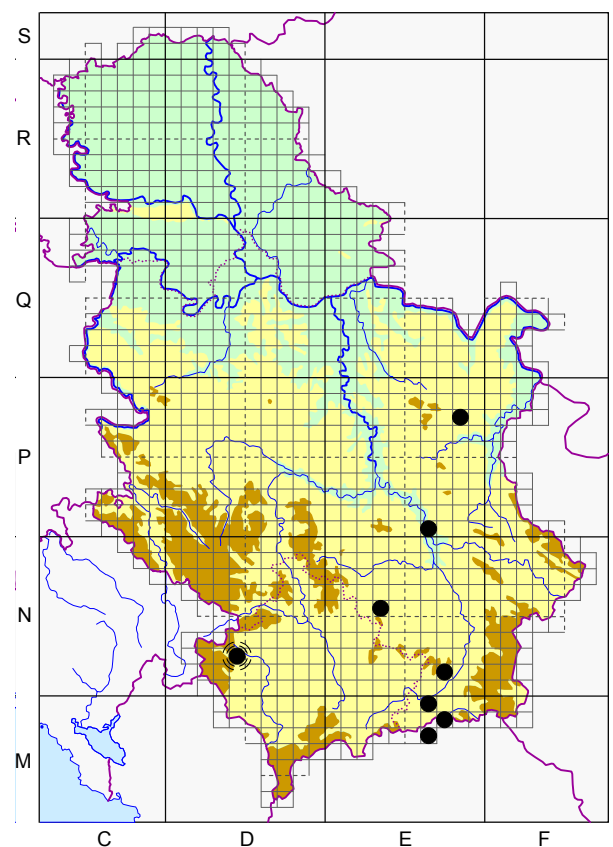

Fig. 24. - Distribution of the species $V$. hymettia Boiss. \& Heldr. in Serbia.
2013); S Serbia Bujanovac: Levosoje, UTM EM69, antimor, stene (leg. Zlatković, B., 28-Apr-2012, det. G. Tomović, 34945 BEOUKEGB); Pljačkavica, UTM EN71, det. G. Tomović, 41168 BEOU, rev. G. Tomović, 07Sep-2013); Radan: Sokolov vis, Veliki krš, UTM EN35, silikat (leg. Niketić, M., Tomović, G. 01-May-2008, det. G. Tomović, BEO); silikat, stene (leg. Niketić, M., Tomović, G. 01-May-2008, det. G. Tomović, 26912 BEOU-KEGB, sub $V$. macedonica, rev. G. Tomović, 23-Sep-2013); Rujan planina: Orljak, Mali Orljak, UTM EM67, krečnjak, kamenjari (leg. Niketić, M., Tomović, G. 04-Apr-2010, 30593 BEOU-KEGB, sub $V$. kitaibeliana, rev. G. Tomović, 07-Sep2013); silikat (leg. Niketić, M., Tomović, G. 04Apr-2010, BEO, rev. G. Tomović, 23-Sep2013); SE Serbia Pčinja - klisura: manastir Prohor Pčinjski, na putu prema, UTM EM78 (leg. Jovanović, S., Vukojičić, S., Pavić, S., 04-Jun-1995, 1637-95 BEOU-KEGB, rev. G. Tomović, 07-Sep-2013); Metohija Prokletije: Peć, UTM DN42, serpentinit, Forsythietum (leg. Soška, Th., 14-Jun-1923, det. G. Tomović, 42004 BEOU).
Viola kitaibeliana Schultes (Fig. 25) Bačka Subotičko-Horgoška peščara: Hajdukovo, Palić - Kasibin šor, UTM DS00, les, higrofilne livade (leg. Niketić, M., Tomoví, G., 12-Apr-2014, det. G. Tomović, BEO); Selevenjska šuma, UTM CS90, pesak, peščara u šumi (leg. Niketić, M., Tomović, G. 12-Apr-2014, det. G. Tomović, BEO); Banat Bela Crkva, UTM EQ37, ruderalna vegetacija (leg. Lakušić, D., 10-Aug-1993, det. D. Lakušić, 322-93 BEOUKEGB, sub $V$. arvensis); Deliblatska peščara (leg. Stevanović, V., det. G. Tomović, 1229 BEOU-KEGB); Čardak, UTM EQ17 (leg. Stevanović, V., Jovanović, S., Lakušić, D., Vukojičić, S. 26-Apr-1997, 4936 BEOU-KEGB); ČardakVrelo, UTM EQ17, pesak, Festucion vaginatae (leg. Stevanović, V. 13-Apr-1997, det. G. Tomović, 4855 BEOU-KEGB); Široka Torina, UTM EQ16 (leg. Stevanović, V. 05-Apr-2008, det. V. Stevanović, 28940 BEOU-KEGB); Deliblato, UTM EQ06 (leg. Grebenščikov, O. 05-May-1945, det. G. Tomović, $V K-003$ BEO); (leg. Grebenščikov, O. 05-May-1946, det. G. Tomović, BEO); (leg. Sigunov, A. 1947, det. G. Tomović, VK-004 BEO); (leg. Soška, Th. May-1929, det. Th. Soška, 41968 BEOU; 41967 BEOU, sub $V$. kitaibeliana stepposa); (leg. Soška, Th. May-

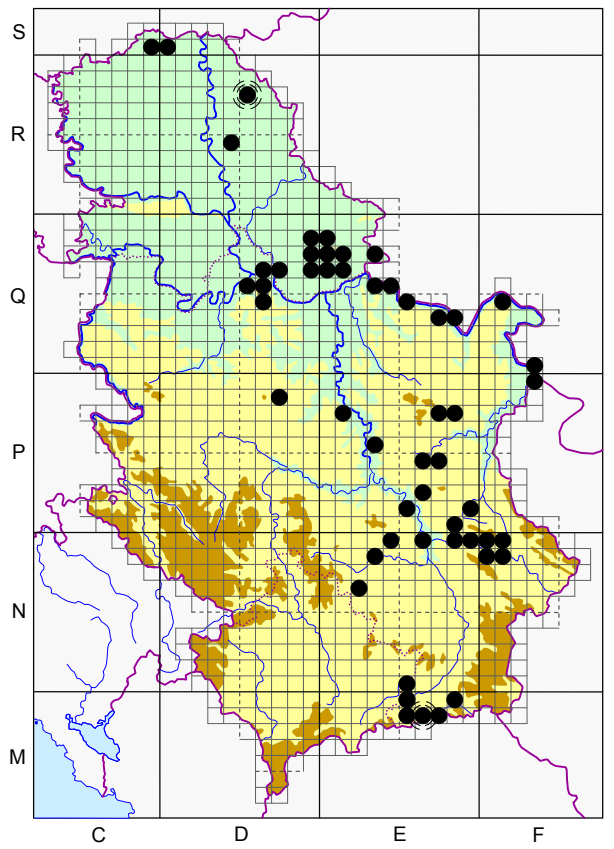

Fig. 25. - Distribution of the species $V$. kitaibeliana Schultes in Serbia. 
1930, det. Th. Soška, 41964 BEOU, sub V. kitaibeliana stepposa); (leg. Soška, Th. 15-Apr-1930, det. Th. Soška, 41961 BEOU, sub V. arvensis var. stepposa, rev. G. Tomović, 07-Sep-2013); Devojački bunar, UTM DQ98, peščarska i stepska vegetacija (leg. Stevanović, V., Jovanović, S. 05-May-1990, det. G. Tomović, 296-90 BEOU-KEGB); stepska vegetacija (leg. Lakušić, D. 16-Apr-1988, det. V. Stevanović, VK-001 BEOU-KEGB); (leg. Stevanović, v. 20-May-1977, det. G. Tomović, 1242 BEOU-KEGB); (leg. Stevanović, V. 29-May-1974, det. V. Stevanović, 41969 BEOU); (leg. studentski herbar May-2010, det. G. Tomović, 33031 BEOU-KEGB); Devojački bunar-Flamunda, UTM DQ98, EQ07 (leg. Nikolić, V., Diklić, N. 29-Apr-1968, det. G. Tomović, 42780 BEO, sub V. arvensis, rev. G. Tomović, 07-Sep-2013); (leg. Nikolić, V., Diklić, N. 26-Apr-1967, det. N. Diklić, 42920 BEO); Flamunda, UTM EQ07 (leg. Lindtner, V. 05-Apr-1933, det. V. Lindtner, 8566 BEO); (leg. Soška, Th. Apr-1943, det. Th. Soška, 8567 BEO, rev, G. Tomović, 23-Sep-2013); vis Jovana Cvijića, UTM EQ07 (leg. Soška, Th. 08-May-1922, det. Th. Soška, 41962 BEOU, sub $V$. arvensis f. trimestris, rev. G. Tomović, 07-Sep-2013); Dolovo-Mali Kravan, UTM DQ97 (leg. Sigunov, A. 04-Apr-1948, det. O. Grebenščikov, 8552 BEO, sub V. banatica, rev. G. Tomović, 23-Sep-2013); Grebenac, UTM EQ17, in arenosis (leg. Soška, Th. 10-Jun-1935, det. G. Tomović, 8563 BEO); Hatarica, UTM EQ16 (leg. Stevanović, V. 29-Mar-1994, det. V. Stevanović, 1148 BEOU-KEGB); Korn bunar, UTM EQ07 (leg. Soška, Th. May-1930, det. Th. Soška, 41963 BEOU, sub $V$. kitaibeliana stepposa); Kremenjak-Grebenac, UTM EQ16, EQ17 (leg. Stevanović, V. 19-May-1972, det. V. Stevanović, 1170 BEOU-KEGB); Mramorak, železnička stanica Mramorak, UTM DQ96, in arenosis (leg. Grebenščikov, O. 23-Apr-1939, det. G. Tomović, VK-002 BEO, sub $V$. stepposa); Šušara, UTM EQ07, otvoreno polje, stepa (leg. Stevanović, V. 11-Apr-1970, det. V. Stevanović, 1293 BEOU-KEGB, sub V. tricolor, rev. G. Tomović, 07-Sep-2013); (leg. Džukić, G. 28-Apr2000, det. G. Džukić, 10693 BEOU-KEGB, sub V. tricolor, rev. G. Tomović, 07-Sep-2013); ŠušaraVeliki Kravan, UTM EQ07 (leg. Sigunov, A. 22-Apr-1948, det. G. Tomović, VK-005 BEO); Velika Tilva, UTM EQ08, peščarska vegetacija (leg. Stevanović, V., Jovanović, S., Lakušić, D. 26-Mar-1990, det. G. Tomović, 18-90 BEOU-KEGB); Zagajička brda, UTM EQ17 (leg. Niketić, M., Stevanović, V., Tomović, G., Vukojičić, S. 12-Apr-2004, BEO); Kikinda, UTM DR57 (leg. Izmajlov 21-Apr-1924, det. Izmajlov, 8553 BEO, sub V. arvensis); Pančevo: Vojlovica, UTM DQ76 (leg. Đelfi, B., det. B. Đelfi, VK-007 BEO, sub V. arvensis, rev. G. Tomović, 23-Sep-2013); Vladimirovci-Alibunar, UTM DQ98, pored puta (leg. Diklić, N. 24-May-1969, det. N. Diklić, 42774, 42775, 42776 BEO, sub V. arvensis, rev. G. Tomović, Feb-2007); Zrenjanin: Melenci, groblje, UTM DR44, groblje (leg. Niketić, M., Tomović, G. 29-Mar-2009, det. G. Tomović, BEO); Šumadija Beograd: Avala, UTM DQ64, DQ65, grabova šuma (leg. Borisavljević, Lj., 18-Apr-1952, det. Lj. Borisavljević, 41982 BEOU, sub V. saxatilis, rev. G. Tomović, 07-Sep-2013); Košutnjak, UTM DQ55 (leg. Grebenščikov, O., Černjavski, R. 17-Mar-1933, det. O. Grebenščikov, VK-008 BEO); okolina, prelozi (leg. Pančić, J., det. J. Pančić, 3295 BEOU (Herb. Panc.), sub V. tricolor); Topčider, pod ćelijom, UTM DQ55 (leg. Pančić, J. 1867, det. J. Pančić, 3318 BEOU (Herb. Panc.), sub $V$. tricolor var. agrestis); Topčiderski park, UTM DQ55, krečnjak, sunčane padine (leg. Grebenščikov, O. 28-Mar-1945, VK-006 BEO, sub V. arvensis, rev. G. Tomović, 23-Sep2013); Veliko selo, UTM DQ66 (leg. Vukojičić, S., Tomović, G., Lazarević, P. 03-Apr-2003, 16334 BEOUKEGB, sub $V$. arvensis, rev. G. Tomović, 23-Sep-2013); Kragujevac: Ramaća, Ramaćki visovi, UTM DP78, silikat (leg. Niketić, M., Tomović, G. 17-Apr-2004, det. M. Niketić, G. Tomović, BEO, rev. G. Tomović, 23-Sep-2013); Pomoravlje Paraćin: Glavica, Strana i Gornje polje, UTM EP35, 150250 m, silikat (leg. Niketić, M., Tomović, G., 24-Apr-2005, det. G. Tomović, 19462 BEOU-KEGB); Velika Morava: Bagrdan klisura, manastir Janković, UTM EP17, silikat, Quercetum frainetto (leg. Niketić, M., Tomović, G. 17-Mar-2007, det. G. Tomović, 24450 BEOU-KEGB); Veliko Gradište: Požeženo, Požeženska peščara, UTM EQ45, pesak (leg. Stevanović, V., Lakušić, D., Vukojičić, S., Tomović, G. 11-Apr-2005, det. G. Tomović, 19369 BEOU-KEGB); (leg. Bogojević, R. 05-Jun-1987, det. R. Bogojević, 3774 BEOU-KEGB); (leg. Bogojević, R. 05-Jun-1987, det. G. Tomović, 3693, 3759 BEOUKEGB); Požeženska peščara, Veliki deo, UTM EQ45, pesak (leg. Niketić, M., Vukojičić, S., Tomović, G., Jakovljević, K., Buzurović, U. 15-Mar-2014, det. G. Tomović, BEO); Zatonje, Zatonjska peščara, UTM EQ35, pesak (leg. Stevanović, V., Lakušić, D., Vukojičić, S., Tomović, G. 11-Apr-2005, det. G. Tomović, 19359 BEOU-KEGB); NE Serbia Bor: Brestovačka banja, UTM EP87 (leg. Pančić, J., Apr-1869, det. J. Pančić, 3327 BEOU (Herb. Panc.), sub V. tricolor var. kitaibeliana); (3331 BEOU (Herb. Panc.), subs V. truncatus); (leg. Pančić, J. 1870, det. J. Pančić, 3211 BEOU (Herb. Panc.), sub V. agrestis); Đerdapska klisura: Boljetin, Boljetinska reka-Dobra, UTM EQ73, EQ83 (leg. Diklić, N. 03-Aug1966, det. N. Diklić, 42921 BEO, sub V. arvensis, rev. G. Tomović, Feb-2007); Golubac, Golubački 
grad, UTM EQ54 (leg. Diklić, N. 20-Apr-1968, det. V. Nikolić, 42792 BEO, sub V. arvensis); Tekija, UTM FQ14 (leg. Pančić, J. May-1872, det. J. Pančić, 3322 BEOU (Herb. Panc.), sub V. tricolor var. arvensis); Malinik: Lazareva reka - kanjon, iznad pećine, UTM EP77, krečnjak, kamenjari (leg. Stevanović, V. 24-Apr-1997, 4973 BEOU-KEGB, sub V. banatica, rev. G. Tomović, 07-Sep-2013); kod pećine, UTM EP77, suve livade (leg. Lakušić, D. 14-Apr-1987, det. D. Lakušić, 724-86 BEOU-KEGB); Negotin: Radujevac, UTM FQ30 (leg. Pančić, J. Jul-1853, det. J. Pančić, 3239 BEOU (Herb. Panc.)); (leg. Soška, Th. 24-Apr-1930, det. Th. Soška, 41965, 41966 BEOU, sub V. kitaibeliana stepposa); Kapu Đalu, UTM FP39, in arenosis (leg. Pančić, J. May-1875, det. J. Pančić, 3213 BEOU (Herb. Panc.), sub V. agrestis); (leg. Soška, Th. 31-Apr-1932, det. G. Tomović, 41993 BEOU, sub V. arvensis); C Serbia Kuršumlija: Kuršumlija-Rudare, $2 \mathrm{~km}$. pre sela Rudare, UTM EN26, $400 \mathrm{~m}$, stene pored puta (leg. Vukojičić, S., Tomović, G., 08-Mar-1997, det. G. Tomović, 8267 BEOU-KEGB); Merošina: Lalinačka slatina, UTM EN69, $50 \mathrm{~m}$, segetalno stanište, aluvijum (leg. Dimitrijević, D. 02-Apr-2007, det. D. Dimitrijević, 28238 BEOU-KEGB); $50 \mathrm{~m}$, halomorfno tlo u fazi olesnjavanja, korovska vegetacija na obradivim površinama, aluvijalni nanosi (leg. Savić, A. 02-Apr-2007, det. A. Savić, 28357 BEOU-KEGB); Prokuplje: Gubetin, kod crkve, UTM EN49, stepski fragment (leg. Niketić, M., Tomović, G. 30-Apr-2008, 26859 BEOU-KEGB, sub V. arvensis, rev. G. Tomović, 23-Sep-2013); Viča selo, Ložđe brdo, UTM EN38, Exp. E, 250 m, voćnjaci (leg. Vukojičić, S., Tomović, G. 03-Sep-1997, det. G. Tomović, 8279 BEOU-KEGB); Exp. SW, 250 m, šuma Quercus frainetto, Carpinus orientalis (leg. Tomović, G. 04-May-1997, det. G. Tomović, 8317 BEOU-KEGB, sub V. tricolor, rev. G. Tomović, 07Sep-2013); E Serbia Aleksinac (leg. Pančić, J., May-1875, det. J. Pančić, 3212 BEOU (Herb. Panc.), sub V. agrestis); (leg. Pančić, J., det. J. Pančić, 3216 BEOU (Herb. Panc.), sub V. arvensis); Žitkovac, Nozrina-Smrdan, UTM EP51, neobrađeni vinogradi (leg. Bogdanović, M. 29-Apr-1973, det. G. Tomović, 42919 BEO); Bela Palanka, UTM FN08 (leg. Pančić, J. 1880, det. G. Tomović, 3200 BEOU (Herb. Panc.), sub Viola sp.); Dol-Donja Glama, UTM FN09, FN19 (leg. Niketić, M., Tomović, G. 01-May2003, 16530 BEOU-KEGB, sub V. arvensis, rev. G. Tomović, 07-Sep-2013); Knjaževac: Svrljiška Topla, UTM EP91, krečnjak, stepski fragment (leg. Niketić, M., Tomović, G. 06-Apr-2008, 26994 BEOUKEGB, sub $V$. arvensis, rev. G. Tomović, 07-Sep-2013); Niš: Jelašnička klisura, UTM EN89, stene (leg. Nikolić, V., Diklić, N. 24-Apr-1965, det. G. Tomović, 42918 BEO); Jelašnica, UTM EN89, krečnjak, stene i kamenjari (leg. Stevanović, V., Lakušić, D. 07-Apr-1990, det. G. Tomović, 62-90 BEOU-KEGB); Pasjača-Sićevo, UTM EN89, EP80 (leg. Soška, Th. May-1932, det. G. Tomović, 41992 BEOU); Sićevačka klisura, manastir Sveta Petka, iznad, UTM EN99 (leg. Nikolić, V., Diklić, N. 25-Apr1965, det. G. Tomović, 42917 BEO); Ostrovica, manastir Sveta Petka, iznad, UTM EN99 (leg. Nikolić, V., Diklić, N. 25-Apr-1965, det. G. Tomović, 42928 BEO); Ozren: Janior, UTM EP62 (leg. Diklić, N. 19-May-1957, det. N. Diklić, 42791 BEO, sub V. arvensis); Pirot: Čiflik, Božin vrh, UTM FN18 (leg. Niketić, M. 04-Apr-2009, BEO, rev. G. Tomović, 23-Sep-2013); Rtanj, UTM EP64, EP74, 1200 m, šibljaci jorgovana (leg. Diklić, N. 08-Jun-1965, det. N. Diklić, 42786 BEO, sub V. arvensis, rev. G. Tomović, Feb-2007); Svrljiške planine: Zeleni vrh, UTM FN09 (leg. Niketić, M. 02-Jun-2008, det. G. Tomović, BEO); S Serbia Bujanovac: Binačka Morava, Končulj klisura, UTM EN50, EM59, kamenjari, čistine u šumi cera (leg. Nikolić, V., Diklić, N., Bogdanović, M., 16-Jun-1975, det. G. Tomović, 42927 BEO); Končulj klisura, ulazna vrata, prosek, UTM EN50, EM59, kamenjari (leg. Nikolić, V. 02-Jun-1966, det. G. Tomović, 42925 BEO); Preševo, UTM EM68, krečnjak, kamenjari u pojasu grabića (leg. Nikolić, V., Diklić, N. 16-May-1975, det. G. Tomović, 42922, 42923, 42924 BEO); iznad, UTM EM68, Exp. W, kamenjari i mešovita hrastova šuma (leg. Nikolić, V., Diklić, N. 22-Apr-1965, det. G. Tomović, 42930 BEO); Ref brdo, UTM EM58, mermer, čistine u šikari grabića (leg. Diklić, N. 31-May1963, det. N. Diklić, 42932 BEO, sub V. arvensis, rev. G. Tomović, Feb-2007); SE Serbia Pčinja klisura: manastir Prohor Pčinjski, UTM EM78 (leg. Stevanović, V., Zlatković, B., Tomović, G., Vukojičić, S., 05-Apr-2004, det. G. Tomović, 22466 BEOU-KEGB); Šajince-Trgovište, UTM EM89, kamenjari (leg. Nikolić, V., Diklić, N. 09-May-1973, det. G. Tomović, 42929 BEO).

Viola orphanidis Boiss. (Fig. 26)

Kosovo Šar-planina: Brezovica-Stojkova kuća-Piribeg, UTM EM06, EM07, kamenjari (leg. Nikolić, V., Diklić, N., Bogdanović, M., 22-Jul-1974, det. G. Tomović, 42985 BEO); Careve livade, oko lovačke kuće, UTM DM96, 1900 m, zona Pinus peuce (leg. Stevanović, V. 26-Jul-1978, det. G. 


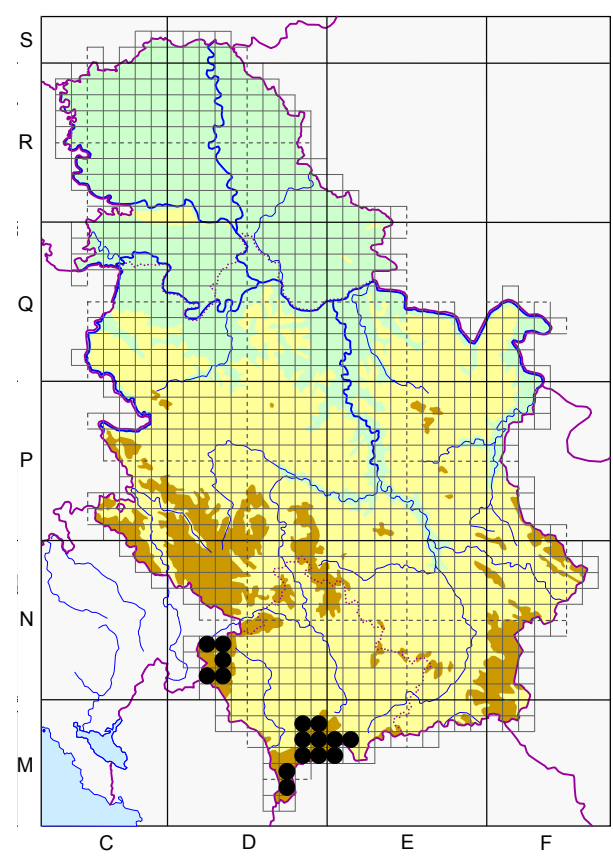

Fig. 26. - Distribution of the species

$V$. orphanidis Boiss. in Serbia.
Tomović, 1665 BEOU-KEGB); Jažinačko jezero, UTM DM96, EM06, 1900 m, zona Pinus peuce (leg. Stevanović, V. 17-Jul-1977, det. G. Tomović, 3189 BEOU-KEGB); Jažinačko jezero-Suva reka, UTM DM96, EM06, 1400-1900 m, mešovita šuma (leg. Nikolić, V., Diklić, N., Mladenović, S. 20-Jul-1978, det. G. Tomović, 42957, 42958, 42959 BEO); Jezerska čuka, ispod, UTM EM06, 2100-2200 m (leg. Jovanović, S., Lakušić, D., Benić, N. 28Jun-1990, det. G. Tomović, 1371-90 BEOUKEGB); Jezerska planina, Bukova glava, UTM DM98, bukova šuma (leg. Nikolić, V. 22Jun-1962, det. G. Tomović, 42980, 42981 BEO; 42960 BEO, sub V. elegantula); Kodža Balkan, Čerenački vrh, UTM DM87, DM97, 1500-2040 m, krečnjak (leg. Nikolić, V., Diklić, N. 19-Jul-1975, det. G. Tomović, 42987, 42988, 42989 BEO); Matos reka - klisura-Gradac, UTM DM88 (leg. Soška, Th. 06-Jun-1923, det. Th. Soška, 41406, 41407 BEOU); Ljuboten, UTM EM07, EM17 (leg. Šuškalović Jul-1902, det. Šuškalović, 41405 BEOU); Bačije, ispod, UTM EM17, bukova šuma (leg. Soška, Th. 04Jul-1914, det. Th. Soška, 41400 BEOU); Oš-

ljak, Golem Bor, UTM DM87, 1900 m, krečnjak (leg. Nikolić, V., Diklić, N. 19-Jul-1975, det. G. Tomović, 42986 BEO); Piribeg, UTM EM06, 2200 m (leg. Stevanović, V. 19-Jul-1989, det. G. Tomović, 3495 BEOU-KEGB); (leg. Stevanović, v., Jovanović, S. 19-Jul-1989, det. G. Tomović, 3364 BEOU-KEGB); (leg. Stevanović, V. 07-Jul-1979, det. G. Tomović, 2120 BEOU-KEGB); Stojkova kuća-Jezerska čuka, UTM EM06, 1200-2600 m, pašnjaci i kamenjari (leg. Nikolić, V., Diklić, N., Mladenović, S. 20-Jul-1977, det. G. Tomović, 42982, 42983 BEO); 1600-2400 m (leg. Nikolić, V., Diklić, N., Mladenović, S. 23-Jul-1980, det. G. Tomović, 42961 BEO, sub V. elegantula); Prevalac, Svinjarski potok, UTM DM96 (leg. Rudski, I. 16-Jul-1930, det. I. Rudski, 41403 BEOU); Metohija Prokletije: Dečanska planina, UTM DN31, bukovo-molikova šuma (leg. Černjavski, P., Rudski, I., Lindtner, V., 15-Jul-1933, det. P. Černjavski, 8405, 8409 BEO); (leg. Lindtner, V. 15-Jul-1933, det. V. Lindtner, 8406 BEO); Hajla, UTM DN23, DN33 (leg. Gošović, S. Aug-1922, det. S. Gošović, 41397 BEOU); Koprivnik, UTM DN31, DN32, $1800 \mathrm{~m}$, in pratis sylvaticus (leg. Grebenščikov, O. 09-Jul-1939, det. O. Grebenščikov, 8407 BEO); Kožnjar, UTM DN21 (leg. Janković, M. Sep-1958, det. G. Tomović, 41399 BEOU); Ločanska Bistrica - dolina, UTM DN21, DN31, bukova šuma (leg. Černjavski, P., Rudski, I., Lindtner, V. 15-Jul-1933, det. N. Diklić, 8411 BEO); (leg. Černjavski, P., Rudski, I., Lindtner, V. 15-Jul-1933, det. P. Černjavski, 8387 BEO); Prilepska planina, UTM DN21 (leg. Janković, M. 1960, det. M. Janković, 41398 BEOU, sub V. elatior, rev. G. Tomović, 21-Dec-2012); Šar-planina: Kobilica, UTM DM86, DM96, 1850 m (leg. Rudski, I. 04-Jul1930, det. I. Rudski, 41404 BEOU); Karaula-Ljubinske uši, UTM DM86, 1900-2300 m (leg. Niketić, M., Tomović, G., Duraki, Š. 08-Jul-2007, det. G. Tomović, 24501 BEOU-KEGB); Konjuška-Crni vrh, Micova ploča, UTM DM96, bukova šuma (leg. Rudski, I. 12-Jul-1930, det. I. Rudski, 41402 BEOU); (leg. Rudski, I. 12-Jul-1930, det. I. Rudski, 8399 BEO); Prevalac-Bistrica, UTM DM96, 1600-2200 m, krečnjak, kamenjari (leg. Nikolić, V., Diklić, N. 23-Jul-1975, det. G. Tomović, 42956 BEO); (leg. Nikolić, V., Diklić, N., Bogdanović, M. 29-Jul-1972, det. G. Tomović, 42990 BEO); Rudoka, UTM DM74, DM75 (leg. Košanin, N. 13-Jul-1911, det. N. Košanin, 41401 BEOU); Šarska reka (leg. Rudski, I. 1939, det. P. Černjavski, 8398 BEO). 
Viola tricolor L. s.l. (Fig. 27)

Banat Kikinda, UTM DR57 (leg. Sretković, G., 10-Aug-1997, det. G. Sretković, 41974 BEOU, sub $V$. elatior, rev. G. Tomović, 07Sep-2013); Vršačke planine: Đakov vrh, UTM EQ29 (leg. Nikolić, V., Diklić, N. 15-Jul1969, det. N. Diklić, 42817 BEO, sub $V$. arvensis var. gracilensis); lugarnica-Velika Čoka, UTM EQ29, EQ39, šuma kitnjaka i lipe (leg. Nikolić, V., Diklić, N. 07-May-1970, det. V. Nikolić, N. Diklić, 42809, 42812 BEO, sub $V$. banatica, rev. G. Tomović, 23-Sep-2013); odmaralište "13. maj" -lugarnica, UTMEQ29, šuma kitnjaka i lipe (leg. Nikolić, V., Diklić, N. 06May-1970, det. V. Nikolić, N. Diklić, 42811 BEO, sub $V$. arvensis, rev. G. Tomović, 23Sep-2013); (42810 BEO, sub V. banatica, rev. G. Tomović, 23-Sep-2013); odmaralište "13. maj" -Velika Čoka, UTM EQ29, EQ39 (leg. Nikolić, V., Diklić, N. 16-Jul-1969, det. G. Tomović, 42770, 42771 BEO, sub V. arvensis, rev. G. Tomović, 23-Sep-2013); planinarski dom Široko bilo, UTM EQ29, proplanci (leg. Nikolić, V., Diklić, N. 26-Jun-1968, det. G. Tomović, $42767 \mathrm{BEO}$, sub $V$. arvensis, rev. G. Tomović, 23-Sep-2013); planinarski dom Široko bilo-Lisičji vrh, UTM EQ29, šuma (leg. Nikolić, V., Diklić, N. 26-Jun-1968, det. G. Tomović, 42765, $42769 \mathrm{BEO}$, sub $V$. arvensis, rev. G. Tomović, 23-Sep-2013); (leg. Nikolić, V., Diklić, N. 26-Jun-1968, det. V. Nikolić, N. Diklić, 42807

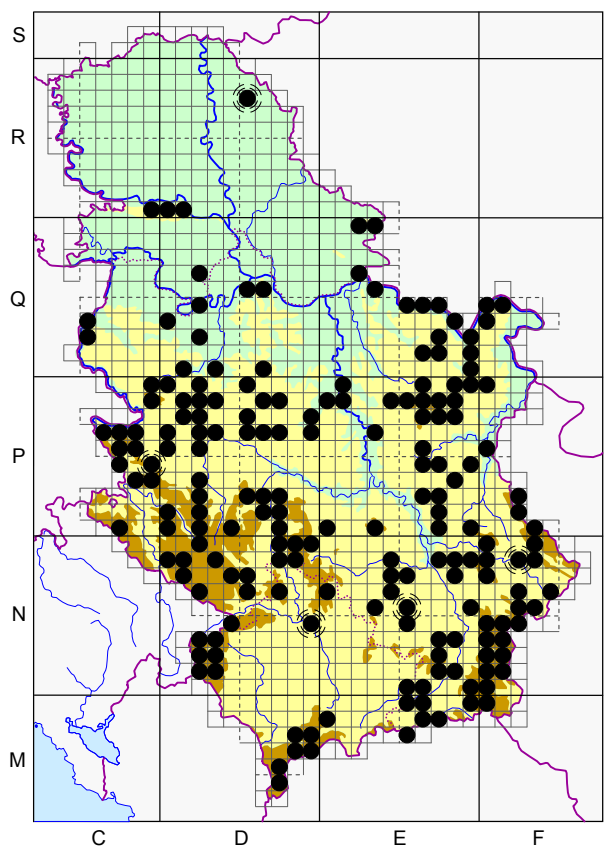

Fig. 27. - Distribution of the species V. tricolor L. s. 1. in Serbia.

BEO, sub $V$. banatica, rev. G. Tomović, 23-Sep-2013); planinarski dom Široko bilo-Vršačka kula, UTM EQ29, hrastova šuma (leg. Nikolić, V., Diklić, N. 26-Jun-1968, det. G. Tomović, 42766, 42001 BEO, sub $V$. arvensis, rev. G. Tomović, 23-Sep-2013); Vršačka kula, UTM EQ29 (leg. Soška, Th. May-1928, det. Th. Soška, 42000 BEOU, sub V. saxatilis, rev. G. Tomović, 07-Sep-2013); Srem Fruška gora: Banstol, UTM DR10, utrine pored puta (leg. Nikolić, V., Diklić, N., 31-May-1968, det. G. Tomović, 42773 BEO, sub V. arvensis); Fruška Gora: Zmajevac, UTM DR00, čistine (leg. Nikolić, V., Diklić, N. 12-Sep-1969, det. V. Nikolić, N. Diklić, 42814 BEO, sub V. banatica, rev. G. Tomović, 23Sep-2013); Fruška gora: Crveni čot, UTM CR90, mešovita listopadna šuma (leg. Nikolić, V., Diklić, N. 06-May-1971, det. V. Nikolić, N. Diklić, 42813 BEO, sub V. banatica, rev. G. Tomović, 23-Sep-2013); Pećinci: Karlovčić, revir, UTM DQ26 (leg. Stanojević, S. 24-Apr-1996, det. S. Stanojević, 42005 BEOU); NW Serbia Jablanik, UTM CP99, Exp. SW, proređena bukova šuma na strmoj kamenitoj podlozi (leg. Nikolić, V., Diklić, N., 25-Jun-1954, det. N. Diklić, 43177 BEO, sub V. tricolor subsp. tricolor, rev. G. Tomović, 23-Sep-2013); planinarski dom, UTM CP99, Exp. E, bukova šuma oko izvora (leg. Nikolić, V., Diklić, N. 26-May-1954, det. N. Diklić, 43083 BEO, sub V. saxatilis subsp. aetolica, rev. G. Tomović, Feb-2007); Exp. N, bukova šuma (leg. Nikolić, V., Diklić, N. 25-May-1954, det. N. Diklić, 43079 BEO, sub $V$. saxatilis, rev. G. Tomović, 23-Sep-2013); Štabulja, UTM CP99, bukova šuma pored puta (leg. Nikolić, V., Diklić, N. 26-May-1954, det. N. Diklić, 43082 BEO, sub V. saxatilis subsp. aetolica, rev. G. Tomović, Feb-2007); vrh, UTM CP99, Exp. E, livade (leg. Nikolić, V., Diklić, N. 24-Jun-1954, det. N. Diklić, 42762 BEO, sub V. arvensis, rev. G. Tomović, 23-Sep-2013); Exp. N, livade (leg. Nikolić, V., Diklić, N. 24-May-1954, det. G. Tomović, 43012 BEO, sub V. macedonica); (leg. Nikolić, V., Diklić, N. 24Jun-1954, det. N. Diklić, 43084 BEO, sub V. saxatilis subsp. aetolica, rev. G. Tomović, Feb-2007); Exp. NE, livade (leg. Nikolić, V., Diklić, N. 24-Jun-1954, det. G. Tomović, 43011 BEO, sub V. macedonica); Loznica: Banja Koviljača, ada, UTM CQ53 (leg. Jurišić, J. Ž. 03-Jun-1912, det. G. Tomović, 8584 BEO); Banja Koviljača-Grabovci, UTM CQ52, ruderalis pored puta (leg. Niketić, M., Tomović, G. 25Apr-2008, 26679 BEOU-KEGB, sub V. macedonica, rev. G. Tomović, 07-Sep-2013); Štira reka, UTM CQ52 (leg. Mitrović, V. 18-May-1998, det. V. Mitrović, 41212 BEOU); Mali Zvornik: Gornja Borina, Borinska reka - klisura, Pešići zaseok, UTM CQ52, voćnjaci (leg. Niketić, M., Tomović, G. 26-Apr2008, 26764 BEOU-KEGB, sub $V$.macedonica, rev. G. Tomović, 07-Sep-2013); Maljen: Bukovi, 
UTM DP18, $868 \mathrm{~m}$ (leg. Tomović, G., Jakovljević, K., Đurović, S., Buzurović, U. 30-May-2014, det. G. Tomović, 40693 BEOU-KEGB, sub V. tricolor subsp. macedonica); Divčibare, UTM DP18, DP28, serpentinit, stanište belog bora, močvarno (leg. Sigunov, A. 19-Jun-1969, det. G. Tomović, 43035 BEO, sub V. macedonica); (leg. Stevanović, V. 28-Jun-1979, det. G. Tomović, 9967 BEOU-KEGB, sub $V$. macedonica); Kraljev sto, ispod, UTM DP28, 967 m, serpentinit, kamenjari (leg. Stevanović, V., Lakušić, D., Veen, P. 22-May-2005, det. G. Tomović, 20072 BEOU-KEGB, sub V. macedonica); Tometino polje, UTM DP27, 880 m, serpentinit, Erico-Pinetum nigrae (leg. Stevanović, V., Jovanović, S., Lakušić, D., Pavić, S. 26-May-1994, det. G. Tomović, 1927-94 BEOU-KEGB, sub V. macedonica); Tometino polje, Pogled ispod, UTM DP27, 603 m, serpentinit, kamenjari (leg. Stevanović, V., Lakušić, D., Veen, P. 22May-2005, det. G. Tomović, 20061 BEOU-KEGB, sub V. macedonica); Medvednik, UTM CP99 (leg. Pančić, J. 1875, det. J. Pančić, 3243 BEOU (Herb. Panc.), sub V. lutea, rev. G. Tomović, 07-Sep-2013); (leg. Pančić, J., det. J. Pančić, 3297 BEOU (Herb. Panc.), rev. G. Tomović, 07-Sep-2013); Mionica: Ribnica - klisura, manastir Pastić, brdo iznad, UTM DP29, krečnjak (leg. Niketić, M., Tomović, G. 29-Mar-2008, det. G. Tomović, 26666 BEOU-KEGB, sub V. macedonica); Povlen: Debelo brdo, UTM CP98 (leg. Pančić, J. Jun-1877, det. G. Tomović, 3193 BEOU (Herb. Panc.), sub Viola sp.); Mali Povlen, UTM CP98, krečnjak (leg. Niketić, M., Tomović, G. 11-Jun-2011, det. G. Tomović, 32258 BEOUKEGB, sub V. macedonica); Suvobor: Rajac, Slavkovica, UTM DP38, Exp. NE, 500 m, granit (leg. Sigunov, A. 20-Apr-1969, det. G. Tomović, 43045 BEO, sub V. macedonica); Vijuljak, UTM DP38, serpentinit (leg. Niketić, M., Jovanović, M. 06-May-2004, det. G. Tomović, BEO); Ub, UTM DQ22, 1926, det. G. Tomović, 41208 BEOU); Valjevo: Gradac reka - kanjon, Lastva, UTM DP18 (leg. Soška, Th. May-1911, det. Th. Soška, 41211 BEOU, sub V. macedonica, rev. G. Tomović, 07-Sep-2013); manastir Ćelije, 1,5 km uzvodno, UTM DP09, Exp. E (leg. Niketić, M. 25-Apr-1992, det. G. Tomović, BEO); Jablanica reka - klisura, manastir Pustinja, UTM CP99, krečnjak, stene (leg. Niketić, $M$. 24-Apr-1992, det. G. Tomović, BEO); Popučke, UTM DQ10 (leg. Lakušić, D. 02-Jul-2006, det. G. Tomović, 21234 BEOU-KEGB, sub V. tricolor subsp. macedonica); Vladimirci: Krnić, Jazovnik, Gomilica kamenolom, UTM DQ03, Exp. S, 140-220 m, kamenolom (leg. Niketić, M., Tomović, G. 04Apr-2004, det. G. Tomović, BEO, sub V. macedonica); Šumadija Aranđelovac: Bukulja, UTM DP59 (leg. Janković, M., 25-May-1953, det. M. Janković, 41206 BEOU, sub V. macedonica); (leg. Janković, M., B. C. 1952, det. G. Tomović, 41983 BEOU, sub V. macedonica); Venčac, UTM DQ60 (leg. Soška, Th. May-1914, det. G. Tomović, 41210 BEOU, sub V. alpestris, rev. G. Tomović, Apr-2007); Brezovac selo, UTM DQ60, serpentinit, kamenjari (leg. Niketić, M., Tomović, G. 21-May-2005, det. G. Tomović, BEO, sub V. macedonica); (19752 BEOU-KEGB); Beograd: Bubanj potok, UTM DQ65, serpentinit, kamenjari pored autoputa (leg. Stevanović, v. 09-Jun-1996, 2441-96 BEOU-KEGB, rev. G. Tomović, 07-Sep-2013); Košutnjak, UTM DQ55 (leg. Janković, M., Mešiček 1952, det. G. Tomović, 41981 BEOU); Rakovica, UTM DQ55 (leg. Bornmüller, J. 1888, det. J. Bornmüller, 41215 BEOU, rev. G. Tomović, Apr-2007); Topčider, UTM DQ55 (leg. Pančić, J., det. J. Pančić, 3294 BEOU (Herb. Panc.), sub V. tricolor; vulgaris); Gornji Milanovac: Brđanska klisura, UTM DP57, serpentinit (leg. Soška, Th. Jun-1926, det. Th. Soška, 41223 BEOU, sub V. saxatilis, rev. G. Tomović, Apr-2007); serpentinit, kamenjari (leg. Stevanović, V., Jovanović, S., Lakušić, D. 19-May-1991, det. G. Tomović, 27-91 BEOU-KEGB); (leg. Stevanović, V., Niketić, M., Vukojičić, S., Tomović, G. 13-Apr-2005, det. G. Tomović, 19395 BEOU-KEGB, sub V. arvensis, rev. G. Tomović, 07-Sep-2013); Rudnik, UTM DP68, Exp. WSW, Quercetum montanum (leg. Diklić, N. 06-Jun-1962, det. G. Tomović, 42997 BEO, sub $V$. macedonica); (Jun-1969, det. G. Tomović, 41228 BEOU); Ljubičevac, Mali Ljubaci, UTM DP68, silikat (leg. Niketić, M. 09-Jun-2013, det. G. Tomović, BEO, sub V. macedonica); Vujan, UTM DP56 (leg. Soška, Th. Jun-1927, det. G. Tomović, 41224 BEOU, sub V. macedonica); manastir Vujan, UTM DP56, 500 m, serpentinit, kamenjari (leg. Lakušić, D. 17-May-2008, 27051 BEOU-KEGB, sub $V$. macedonica); Knić: Borač, Borački krš, UTM DP66, gabro, stene (leg. Stevanović, V. 08-Jun-2003, det. G. Tomović, 22311 BEOU-KEGB, sub V. macedonica); silikat (leg. Niketić, M., Tomović, G. 17-Apr2004, det. G. Tomović, BEO, sub V. macedonica); Bumbarevo Brdo, na ulazu u selo, UTM DP76, 
mezofilna livada (leg. Niketić, M., Buzurović, U. 04-Jun-2014, det. G. Tomović, BEO); Kragujevac, jendeci (leg. Pavlović 1873, det. Pavlović, 42003 BEOU); Bresnica, ulica Užičke Republike, UTM DP96, 190 m, pored kolovoza (leg. Marinković, D. 27-May-1997, det. V. Stevanović, 41227 BEOU, sub V. arvensis, rev. G. Tomović, Apr-2007); Dobrača, Seničko polje, UTM DP78 (leg. Rudski, I. 24-Jul1938, 8579 BEO, rev. G. Tomović, 23-Sep-2013); Trmbas, UTM DP97 (leg. Rudski, I. 23-May-1937, 8577 BEO, rev. G. Tomović, 23-Sep-2013); Lajkovac: Vračević, UTM DQ30, grabova šima (leg. Černjavski, P. 08-Jul-1948, det. P. Černjavski, 8562 BEO, sub V. arvensis, rev. G. Tomović, 23-Sep2013); Obrenovac: TE "Nikola Tesla", kaseta I, UTM DQ24, deponije pepela i šljake (leg. Kostić, Z. 22-Apr-2000, det. Z. Kostić, 41205 BEOU, rev. G. Tomović, Apr-2007); Pomoravlje Svilajnac: Miljkov manastir, UTM EP19, silikat (leg. Niketić, M., Tomović, G. 28-Apr-2003, det. G. Tomović, 16408 BEOU-KEGB, sub $V$. arvensis); Velika Morava: Bagrdan klisura, manastir Janković, UTM EP18, silikat, Quercetum confertae (leg. Niketić, M., Tomović, G. 17-Mar-2007, det. G. Tomović, 24448 BEOU-KEGB, sub V. macedonica); Brzan, Đunis mala, UTM EP08, silikat (leg. Niketić, M. 09Apr-2010, det. M. Niketić, BEO, sub V. macedonica, rev. G. Tomović, 23-Sep-2013); Veliko Gradište: Gradištanska peščara (leg. Bogojević, R., 10040 BEOU-KEGB, rev. G. Tomović, 07Sep-2013); Ram, UTM EQ26 (leg. Pančić, J., det. J. Pančić, 3298 BEOU (Herb. Panc.), rev. G. Tomović, 07-Sep-2013); Srebrno jezero, UTM EQ35 (leg. Bogojević, R. 01-Apr-1987, 10063 BEOUKEGB, sub $V$. arvensis, rev. G. Tomović, 07-Sep-2013); NE Serbia Beljanica, UTM EP58 (leg. Pančić, J., Jul-1863, det. J. Pančić, 3251 BEOU (Herb. Panc.), sub V. macedonica); (leg. Pančí, J. 1872, det. J. Pančić, 3325 BEOU (Herb. Panc.), sub V. tricolor var. bannatica, rev. G. Tomović, 07-Sep-2013); Busovata, UTM EP58, $1000 \mathrm{~m}$ (leg. Lindtner, V. 24-May-1948, det. G. Tomović, VM-002, VM-003, VM-004 BEO, sub V. macedonica); (leg. Popović ? 25-Jun-1950, det. G. Tomović, VM-001 BEO, sub V. macedonica); Resavska reka - klisura, UTM EP48, EP58, Exp. SW, 400 m, krečnjak, FagoColurnetea mixtia, 07-Jul-1998, det. G. Tomović, 41196 BEOU); krečnjak, stene (leg. Nikolić, V., Diklić, N. 12-Jun-1976, det. N. Diklić, 43174, 43175 BEO, sub V. tricolor subsp. tricolor, rev. G. Tomović, 23Sep-2013); (20-Jul-1997, det. G. Tomović, 41194 BEOU, rev. G. Tomović, Apr-2007); Suvaja reka klisura, 12-Jul-1998, det. G. Tomović, 41195 BEOU); Boljevac: Krivi vir, UTM EP65, 30-Jun-1955, 41977 BEOU, rev. G. Tomović, 07-Sep-2013); Bor: Brestovačka banja, Kljancu Mare, UTM EP87 (leg. Pančić, J. 1868, det. J. Pančić, 15010 BEOU (Herb. Panc.), sub V. lactea, rev. G. Tomović, 07-Sep2013); Crni vrh, UTM EP78, Exp. S, livade u bukovoj šumi (leg. Nikolić, V. 27-May-1955, det. G. Tomović, 43001 BEO, sub V. macedonica); (leg. Pančić, J., det. J. Pančić, 3296 BEOU (Herb. Panc.), rev. G. Tomović, 07-Sep-2013); Krivelj, UTM EP88 (leg. Soška, Th. 24-Jun-1923, det. G. Tomović, 41200 BEOU, sub V. macedonica); Stol, UTM EP99 (leg. Pančić, J. 1876, det. G. Tomović, 3192 BEOU (Herb. Panc.), sub Viola sp.); (leg. Soška, Th. 26-Jun-1923, det. G. Tomović, 41197 BEOU, sub V. macedonica); Lučka reka, gornja strana, UTM EP99, livade (leg. Nikolić, v. 24-May-1955, det. G. Tomović, 43000 BEO, sub V. macedonica); Luka, UTM EP99 (leg. Pančić, J. 1876, det. G. Tomović, 3191 BEOU (Herb. Panc.), sub Viola sp.); vrh, UTM EP99, 1156 m, kamenjari (leg. Nikolić, V., Diklić, N. 29-Jun-1961, det. G. Tomović, 43018 BEO, sub V. macedonica); Veliki krš, UTM EP89, 1149 m, kamenjari (leg. Benić, N. 13-Jul-1990, 1730-90 BEOU-KEGB, rev. G. Tomović, 07-Sep-2013); isušena močvara (leg. Benić, N. 15-Jul-1990, det. G. Tomović, 1795-90 BEOU-KEGB, sub V. tricolor subsp. macedonica); livade, bukova šuma (leg. Benić, N. 06-Jul-1990, det. G. Tomović, 1763-90 BEOU-KEGB, sub V. tricolor subsp. macedonica); stene i kamenjari (leg. Benić, N. 09-Jul-1990, det. G. Tomović, 178990 BEOU-KEGB, sub V. tricolor subsp. macedonica); (leg. Benić, N. 07-Jun-1991, det. G. Tomović, 1456-91 BEOU-KEGB, sub V. macedonica); greben, UTM EP89, Exp. N, pašnjaci (leg. Diklić, N. 24Jun-1961, det. N. Diklić, 43066 BEO, sub V. saxatilis subsp. macedonica); Deli Jovan: Ljiljak, vrh, UTM FP09, kamenjari (leg. Nikolić, V. 15-Jun-1956, det. G. Tomović, 42998 BEO, sub V. macedonica); Veliki Goli vrh, UTM EQ90, 1037 m (leg. Niketić, M., Tomović, G. 02-Jun-2005, det. G. Tomović, BEO, sub V. macedonica); kota 1027, UTM EP99, 1027 m (leg. Niketić, M., Tomović, G. 02-Jun-2005, det. G. Tomović, BEO, sub V. macedonica); Đerdapska klisura: Brnjica, UTM EQ64, stene pored puta 
(leg. Nikolić, V., Diklić, N. 28-Sep-1966, det. G. Tomović, 42806 BEO, sub V. banatica, rev. G. Tomović, 23-Sep-2013); Brnjička reka - kanjon, UTM EQ64, krečnjak (leg. Stevanović, V., Vukojičić, S., Tomović, G., "ENDEMIT" 18-Jul-2001, det. G. Tomović, 13448 BEOU-KEGB, sub V. macedonica); Donji Milanovac (leg. Nikolić, V., Diklić, N. 04-Aug-1966, det. V. Nikolić, N. Diklić, 42805 BEO, sub V. banatica, rev. G. Tomović, 23-Sep-2013); Dobra, 10 km istočno, UTM EQ83, krečnjak, pored puta (leg. Niketić, M., Tomović, G. 14-Apr-2008, 26844 BEOU-KEGB, sub V. banatica, rev. G. Tomović, 07Sep-2013); Dobra-Lepenski vir, UTM EQ74, EQ83 (leg. Stevanović, V., Vukojičić, S., Tomović, G., Lazarević, P. 20-Apr-2002, 14776 BEOU-KEGB, sub V. arvensis, rev. G. Tomović, 07-Sep-2013); Porečka reka, UTM EQ92 (leg. Nikolić, V., Diklić, N. 04-Aug-1966, det. N. Diklić, 43064 BEO, sub V. saxatilis subsp. macedonica); Veliki Greben, UTM EQ91, EQ92, pored puta (leg. Nikolić, V., Diklić, N. 24-Sep-1966, det. N. Diklić, 43062 BEO, sub V. saxatilis subsp. macedonica); (leg. Pančić, J.? Apr-1875, det. J. Pančić, 15006 BEOU (Herb. Panc.), rev. G. Tomović, 07-Sep-2013); Veliki Greben, Tri Štubleja-Cigansko groblje, pored puta, UTM EQ92, livade (leg. Nikolić, V. 20-Jun-1956, det. G. Tomović, 42999 BEO, sub V. macedonica); Golo brdo, UTM FQ14 (leg. Nikolić, V., Diklić, N. 18-Jun1969, det. N. Diklić, 42787 BEO, sub V. arvensis, rev. G. Tomović, 23-Sep-2013); Golubac, UTM EQ54, krečnjak, Asplenietea rupestris (leg. Stevanović, V., Lakušić, D. 07-May-1995, det. G. Tomović, 8095 BEOU-KEGB, sub V. macedonica); (leg. Bogojević, R., 3792 BEOU-KEGB, sub V. arvensis, rev. G. Tomović, 07-Sep-2013); Golubački grad, UTM EQ54 (leg. Nikolić, V., Diklić, N. 19-Apr-1968, det. V. Nikolić, 43059, 43060, 43061 BEO, sub V. saxatilis subsp. aetolica, rev. G. Tomović, Feb-2007); Miroč, Konjska glava, UTM FQ03, 500 m, krečnjak, Syringo-Carpinion orientalis (leg. Stevanović, V., Lakušić, D., Niketić, M. 10-May-1996, 17-96 BEOU-KEGB, rev. G. Tomović, 07-Sep-2013); Krš pored Golubinjske reke, UTM EQ92, Exp. S, Syringetum (leg. Diklić, N. 16-May-1955, det. N. Diklić, 43074 BEO, rev. G. Tomović, 23-Sep-2013); (leg. Diklić, N. 20-Jun-1955, det. N. Diklić, 43073 BEO, sub V. saxatilis, rev. G. Tomović, 23-Sep-2013); Petrovo selo, UTM FQ14, krečnjak (leg. Stevanović, V., Jovanović, S., Lakušić, D., Pavić, S. 30-Sep-1994, 1759-94 BEOU-KEGB, sub V. banatica, rev. G. Tomović, 07-Sep2013); Ploče, UTM FQ04 (leg. Stevanović, V., Vukojičić, S., Tomović, G., Lazarević, P 20-Apr-2002, det. G. Tomović, 14782 BEOU-KEGB, sub $V$. macedonica); Štrbac, Mali Štrbac, UTM FQ04, $600 \mathrm{~m}$, krečnjak, stene (leg. Vukojičić, S., Tomović, G., "ENDEMIT" 16-Jul-2001, det. G. Tomović, 13432 BEOUKEGB, sub V. macedonica); Veliki Štrbac, UTM FQ03, krečnjak, stene i kamenjari (leg. Lakušić, D. 08May-1995, det. G. Tomović, 145-95 BEOU-KEGB, sub V. macedonica); (leg. Stevanović, V., Vukojičić, S., Tomović, G., Lazarević, P 20-Apr-2002, 14824 BEOU-KEGB, rev. G. Tomović, 07-Sep-2013); (leg. Stevanović, V., Vukojičić, S., Tomović, G., Lazarević, P 21-Apr-2002, 14879 BEOU-KEGB, sub V. arvensis, rev. G. Tomović, 07-Sep-2013); Veliki Štrbac, glavni vis, UTM FQ03, Exp. S, 550 m, retka mešovita šuma (leg. Diklić, N. 20-May-1955, det. G. Tomović, 43028 BEO, sub V. macedonica); Veliki Štrbac, lugarnica-vrh, UTM FQ03, šuma (leg. Nikolić, V., Diklić, N. 25-Apr-1968, det. V. Nikolić, 43057 BEO, sub V. saxatilis subsp. aetolica, rev. G. Tomović, Feb-2007); Veliki Štrbac, Pecka bara-lugarnica, UTM FQ03 (leg. Nikolić, V., Diklić, N. 19-May-1967, det. N. Diklić, 43065 BEO, sub V. saxatilis subsp. macedonica); Veliki Štrbac, vrh, UTM FQ03, kamenjari sa proređenim šibljakom (leg. Diklić, N., Pajović, M. 26-Jul-1955, det. N. Diklić, 43002 BEO, rev. G. Tomović, 23-Sep-2013); Tekija, UTM FQ14 (leg. Pančić, J. May-1872, det. J. Pančić, 3307 BEOU (Herb. Panc.), sub V. tricolor var. bannatica, rev. G. Tomović, 07-Sep-2013); Jeremijin potok, UTM FQ14, livade (leg. Nikolić, V., Diklić, N. 15-Jun-1969, det. N. Diklić, 42788 BEO, sub V. arvensis, rev. G. Tomović, 23-Sep-2013); kod starog mosta, UTM FQ14, krečnjak, ruderalis (leg. Stevanović, V., Jovanović, S., Lakušić, D., Pavić, S. 30-Sep-1994, 1665-94 BEOU-KEGB, rev. G. Tomović, 07-Sep-2013); Kovilovski potok, UTM FQ14 (leg. Nikolić, V., Diklić, N. 24-Apr-1968, det. V. Nikolić, 43058 BEO, sub V. saxatilis subsp. aetolica, rev. G. Tomović, Feb-2007); (leg. Nikolić, V., Diklić, N. 26-Jun-1967, det. G. Tomović, 43063 BEO, sub V. saxatilis subsp. macedonica); Kučaj: Lisac, Veliki Lisac, UTM EP68 (leg. Niketić, M. 14-Jun-1995, det. G. Tomović, BEO, sub $V$. tricolor subsp. macedonica); Veliki Lisac, izvor Ravne reke, UTM EP68 (leg. Niketić, M. 14-Jun-1995, det. G. Tomović, BEO, sub V. tricolor subsp. macedonica); Majdanpek, UTM EQ71 (leg. Pančić, J. 
1875, det. J. Pančić, 3189 BEOU (Herb. Panc.), sub V. speciosa, rev. G. Tomović, 23-Sep-2013); (8580 BEO, rev. G. Tomović, 23-Sep-2013); (leg. Košanin, N. May-1925, 41198 BEOU, sub V. saxatilis, rev. G. Tomović, Apr-2007); (leg. Pančić, J. Jul-1877, det. J. Pančić, 3310 BEOU (Herb. Panc.), rev. G. Tomović, 07-Sep-2013); (leg. Pančić, J. 1879, det. G. Tomović, 3199 BEOU (Herb. Panc.), sub Viola sp.); Debeli lug, UTM EQ71, duž potoka kraj šume, 29-Apr-1947, det. G. Tomović, VM-015 BEO); Exp. SW, mešovita hrastovo-grabova sastojina (leg. Černjavski, P. 26-Apr-1947, det. G. Tomović, VM-006 BEO, sub V. macedonica); (18-Aug-1947, BEO, rev. G. Tomović, 23-Sep-2013); (leg. Černjavski, P. 27-Apr1944, det. P. Černjavski, 8551 BEO, sub V. arvensis, rev. G. Tomović, 23-Sep-2013); Krst Trostura, UTM EQ71, krečnjak, 26-Apr-1947, det. G. Tomović, VM-010 BEO); krš, UTM EQ71, 18-Aug-1947, det. G. Tomović, VM-013 BEO); (27-Apr-1947, det. G. Tomović, VM-007 BEO); Pek, pored, UTM EQ71, krečnjak, stene (leg. Niketić, M., Tomović, G. 09-Jul-2009, 30163 BEOU-KEGB, sub V. banatica, rev. G. Tomović, 07-Sep-2013); (23-Jul-1947, det. G. Tomović, VM-011 BEO); Majdanpečka Domena, UTM EQ71, hrastova šuma, 11-Jul-1947, det. G. Tomović, VM-009 BEO); mala uvala vrbaka (leg. Gajić, M. 24-Sep-1957, det. G. Tomović, VM-012 BEO, sub V. macedonica); Breza, UTM EQ61, krečnjak, pukotine stena, 18-Jul-1947, det. G. Tomović, VM-005 BEO); Čerdez čuka, UTM EQ61, hrastova šuma (leg. Černjavski, P. 25-Jul-1947, det. G. Tomović, VM-008 BEO, sub V. macedonica); Starica, glavni greben, UTM EQ72, krečnjak (leg. Niketić, M. 04-Jul-2010, det. G. Tomović, BEO, sub V. macedonica); greben, UTM EQ72, Exp. SE, 500 m, livade (leg. Diklić, N. 19-Jul-1968, det. G. Tomović, 43047 BEO, sub V. macedonica); Malinik: Lazareva reka - kanjon, UTM EP67, EP77, krečnjak (leg. Lakušić, D., Tomović, G. 18-May-2005, 19730 BEOU-KEGB, sub V. tricolor subsp. macedonica, rev. G. Tomović, 07-Sep-2013); (leg. Lakušić, D. Jul-1986, det. D. Lakušić, 491-86 BEOUKEGB, sub $V$. arvensis, rev. G. Tomović, 07-Sep-2013); (Jul-1986, 492-89 BEOU-KEGB, sub $V$. banatica, rev. G. Tomović, 07-Sep-2013); (10-Jul-1985, 81-85 BEOU-KEGB, det. G. Tomović, 07-Sep2013); (leg. Lakušić, D. 01-May-1987, det. D. Lakušić, 138-87 BEOU-KEGB, sub V. banatica, rev. G. Tomović, 07-Sep-2013); (leg. Nikolić, V., Diklić, N., Bogdanović, M. 18-Jun-1972, det. G. Tomović, 43023 BEO, sub V. macedonica); (leg. Stevanović, V. 06-Jun-1983, det. G. Tomović, 1734 BEOU-KEGB, sub $V$. macedonica); iznad pećine, UTM EP77, krečnjak, kamenjari (leg. Stevanović, V. 24-Apr-1997, 4973 BEOU-KEGB, sub V. banatica, rev. G. Tomović, 07-Sep-2013); vrh, UTM EP77, 41955 BEOU, rev. G. Tomović, 07-Sep-2013); Zaječar: Vrška čuka, UTM FP05 (leg. Pančić, J. May-1875, det. J. Pančić, 3309 BEOU (Herb. Panc.), rev. G. Tomović, 07-Sep-2013); (leg. Pančić, J., det. J. Pančić, 3250 BEOU (Herb. Panc.), sub V. macedonica); greben-vrh, UTM FP05, 650 m, krečnjak (leg. Nikolić, V., Diklić, N. 24-Jun-1975, det. V. Nikolić, N. Diklić, 42815 BEO, sub V. banatica, rev. G. Tomović, 23-Sep-2013); Žagubica: Mlava reka - vrelo-Buk, UTM EP69, čistine u pojasu bukove šume (leg. Diklić, N. 23Sep-1969, det. N. Diklić, 42785 BEO, sub V. arvensis, rev. G. Tomović, 23-Sep-2013); (leg. Diklić, N. 23Sep-1969, det. N. Diklić, 43005 BEO, sub V. dacica, rev. G. Tomović, Feb-2007); (leg. Diklić, N. 23-Sep1969, det. N. Diklić, 43006 BEO, sub V. dacica, rev. G. Tomović, 23-Sep-2013); W Serbia Čačak: Tučkovo, Vrčanska reka - klisura, UTM DP26, DP36, serpentinit (leg. Stevanović, V., Niketić, M., Vukojičić, S., Tomović, G., 30-Apr-2004, 18541 BEOU-KEGB, sub V. arvensis, rev. G. Tomović, 07-Sep2013); Čemerno, UTM DP52 (leg. Pančić, J., det. J. Pančić, 15013 BEOU (Herb. Panc.), sub V. macedonica); Ivanjica: Panjica reka - klisura, UTM DP23, Orno-Ostryetum, otvorene stene (leg. Stanić, S., Benić, N. 27-Jul-1990, 2760-90 BEOU-KEGB, rev. G. Tomović, 07-Sep-2013); Javor, proplanci u bukovoj šumi (leg. Nikolić, V. 30-Jul-1981, det. G. Tomović, 43052 BEO, sub $V$. macedonica); (leg. Pančić, J., det. J. Pančić, 3241 BEOU (Herb. Panc.), sub V. lutea, rev. G. Tomović, 07-Sep-2013); Javor-Kladnica, UTM DP20, DP21, livade (leg. Nikolić, V. 30-Jul-1981, det. G. Tomović, 43013 BEO, sub V. macedonica); Kosjerić, UTM DP17 (leg. Ostojić, D. 10-Jun-1997, det. G. Tomović, 7111 BEOU-KEGB, sub V. macedonica); Mokra gora: Ivica, UTM CP75 (leg. Pančić, J. Jun-1877, det. G. Tomović, 3194 BEOU (Herb. Panc.), sub Viola sp.); Šargan, UTM CP85, serpentinit (leg. Soška, Th. Jun-1926, det. Th. Soška, 41219 BEOU, sub V. saxatilis, rev. G. Tomović, Apr-2007); Mučanj, UTM DP22 (leg. Vukojičić, S. May-2003, det. G. Tomović, 28863 BEOU-KEGB, sub $V$. 
macedonica); Katići, UTM DP22, stene, kamenjari, šuma (leg. Lakušić, D. 17-Oct-1990, 2845-90 BEOUKEGB, rev. G. Tomović, 07-Sep-2013); Nova Varoš: Rasadi, UTM DP01, na putu (leg. llić, S. 15Jun-1929, det. G. Tomović, 41233 BEOU, sub V. macedonica); oranice (leg. llić, S. 23-Jun-1929, det. S. Ilić, 41236 BEOU, sub V. alpestris, rev. G. Tomović, Apr-2007); (leg. llić, S. 25-Jun-1929, det. S. Ilić, 41232 BEOU, sub V. alpestris, rev. G. Tomović, Apr-2007); Svijetnjak, UTM DP01 (leg. llić, S. 20May-1929, det. S. Ilić, 41235 BEOU, sub V. alpestris, rev. G. Tomović, Apr-2007); OvčarskoKablarska klisura, UTM DP36 (leg. Nikolić, V., det. G. Tomović, 10852 BEO, sub V. macedonica); (leg. Ostojić, D. 22-Apr-1998, det. G. Tomović, 10852 BEOU-KEGB, sub V. macedonica); (leg. Soška, Th. Jun-1926, det. Th. Soška, 41222 BEOU, sub V. saxatilis, rev. G. Tomović, Apr-2007); Kablar, UTM DP36, krečnjak, stene (leg. Pančić, J., det. J. Pančić, 15055 BEOU (Herb. Panc.), sub V. chrysantha, rev. G. Tomović, 07-Sep-2013); Ovčar, UTM DP36, krečnjak (leg. Stevanović, V., Niketić, M., Vukojičić, S., Tomović, G. 13-Apr-2005, det. G. Tomović, 19384 BEOU-KEGB, sub V. tricolor subsp. macedonica); Ovčar banja, UTM DP36 (leg. Diklić, N., Bogdanović, M. 20-May-1974, det. G. Tomović, 43024 BEO, sub V. macedonica); Požega: Jelen Do, Krš, UTM DP25, krečnjak (leg. Niketić, M., Tomović, G. 11Apr-2009, det. G. Tomović, BEO, sub V. macedonica); Tara planina, 41226 BEOU, rev. G. Tomović, Apr-2007); Kaluđerske bare, UTM CP86, krečnjak (leg. Stevanović, V., Niketić, M., Tomović, G. 15-Jul1998, 12766 BEOU-KEGB, rev. G. Tomović, 07-Sep-2013); Kremna, UTM CP85, ivica jelove i smrčeve šume (leg. Diklić, N. 16-Sep-1966, det. G. Tomović, 43048 BEO, sub V. macedonica); serpentinit, kamenjari (leg. Diklić, N., Bogdanović, M. 19-May-1974, det. G. Tomović, 43025 BEO, sub V. macedonica); Karačica reka - klisura, UTM CP85, c. 680 m, serpentiniti, kamenjari (leg. Niketić, M., Tomović, G., Buzurović, U. 10-May-2014, det. G. Tomović, 40635 BEOU-KEGB, sub V. tricolor subsp. macedonica); Kremanska kotlina, UTM CP85, serpentinit (leg. Stevanović, V., Jovanović, S., Pavić, S. 30May-1994, det. G. Tomović, 1990-94 BEOU-KEGB, sub V. macedonica); Ljuto polje, UTM CP75 (leg. Košanin, N., Soška, Th. 1912, det. G. Tomović, VM-023 BEO, sub V. macedonica); (leg. Soška, Th. Jun-1912, det. Th. Soška, 41216 BEOU, sub V. alpestris, rev. G. Tomović, Apr-2007); Ljuto poljeKarajića bare, UTM CP75, CP76, tresave i Nardetum-i (leg. Stevanović, V., Jovanović, S., Vukojičić, S. 08-Jul-2000, 14626 BEOU-KEGB, sub V. arvensis, rev. G. Tomović, 07-Sep-2013); Mitrovac, UTM CP76, livade (leg. Diklić, N., Nikolić, V. 16-Jul-1960, det. G. Tomović, 43075 BEO, sub V. macedonica); Crveni potok - tresava, UTM CP76 (leg. Stevanović, V., Jovanović, S., Pavić, S. 28-May-1994, det. G. Tomović, 1980-94 BEOU-KEGB, sub V. macedonica); Rača reka - kanjon, Borovo brdo, UTM CP86, livade (leg. Diklić, N., Rakin, M. 17-Jul-1963, det. G. Tomović, 43078 BEO, sub V. macedonica); donji deo, UTM CP86 (leg. Diklić, N., Bogdanović, M. 18-May-1974, det. G. Tomović, 43014 BEO, sub $V$. macedonica); Zaovine, UTM CP75 (leg. Terzić Jun-1920, det. Terzić, 41220 BEOU, sub V. saxatilis, rev. G. Tomović, Apr-2007); jezero, iznad, UTM CP75, pašnjaci, kultura krompira (leg. Stevanović, V., Jovanović, S. 11-Sep-1996, det. G. Tomović, 2962-96 BEOU-KEGB, sub V. macedonica); Užice (leg. Pančić, J., det. J. Pančić, 15011 BEOU (Herb. Panc.), sub V. tricolor var. vulgaris, rev. G. Tomović, 07Sep-2013); (leg. Urošević, K., 41217 BEOU, sub V. saxatilis, rev. G. Tomović, Apr-2007); Buar-Gornja Pora, UTM DP05, 660 m (leg. Vukojičić, S. 01-May-1996, det. S. Vukojičić, 41225 BEOU, rev. G. Tomović, Apr-2007); Đetina reka - klisura, Zabučje, UTM DP05, krečnjak, in saxosis (leg. Soška, Th. Jun-1926, det. Th. Soška, 41221 BEOU, sub V. saxatilis, rev. G. Tomović, Apr-2007); Jelova gora, Đakov kamen, ka Kadinjači, UTM DP06, 900 m (leg. Vukojičić, S. 06-Jul-2014, det. G. Tomović, 17076 BEOU); Krčagovo, UTM DP05 (leg. Košanin, N. 15-Apr-1889, det. N. Košanin, 41214 BEOU, rev. G. Tomović, Apr-2007); Zlatibor, serpentinit (leg. Jovanović, S. 01-Jun-1989, det. G. Tomović, 120-89 BEOU-KEGB, sub V. tricolor subsp. macedonica); (leg. Pančić, J. 1875, det. J. Pančić, 3242 BEOU (Herb. Panc.), sub V. lutea, rev. G. Tomović, 07-Sep-2013); Dobroselica - Ljubiš, UTM CP93, 1102 m (leg. Tomović, G., Jakovljević, K., Đurović, S., Buzurović, U. 29-May-2014, det. G. Tomović, 40690 BEOUKEGB, sub V. tricolor subsp. macedonica); Panjak selo, UTM CP74, serpentiniti, kamenjari (leg. Niketić, M., Tomović, G., Buzurović, U. 10-May-2014, det. G. Tomović, 40636 BEOU-KEGB, sub V. tricolor subsp. macedonica); površ, UTM CP94, serpentinit, Halascyetalia sendtneri (leg. Jovanović, S., Lakušić, 
D., Glišić, O., Tomović, G. 22-May-1996, 189-96 BEOU-KEGB, rev. G. Tomović, 07-Sep-2013); Ribnica, UTM CP93, livade (leg. Košanin, N. 06-Aug-1930, det. N. Košanin, 41218 BEOU, sub V. saxatilis, rev. G. Tomović, Apr-2007); (leg. Vukojičić, S. 28-Apr-2006, det. G. Tomović, 28862 BEOU-KEGB, sub $V$. macedonica); Stublo, put ka manastiru Uvac i manastiru Dubrava, UTM CP83, serpentinit (leg. Vukojičić, S., Tomović, G., Zlatković, B., Jakovljević, K., Kabaš, E. 10-Jun-2012, det. G. Tomović, 35270 BEOU-KEGB, sub V. macedonica); Zvezda: Dikava, UTM CP66 (leg. Pančić, J. 1875, det. J. Pančić, 3244, 3245 BEOU (Herb. Panc.), sub V. lutea, rev. G. Tomović, 07-Sep-2013); C Serbia Goč: Mekušnica reka - dolina, UTM DP72, livade u dolini reke (leg. Đelfi, B., 06-Aug-1936, det. G. Tomović, VM-014 BEO, sub V. macedonica); Ibarska dolina (leg. Košanin, N. 25-Apr-1928, det. G. Tomović, 41239 BEOU, sub V. macedonica); Bogutovac - Ušće, UTM DP62, 286 m (leg. Tomović, G., Jakovljević, K., Đurović, S., Buzurović, U. 28-May-2014, det. G. Tomović, 40656 BEOU-KEGB, sub $V$. tricolor subsp. macedonica); Maglić, Maglić grad, UTM DP62, serpentinit (leg. Niketić, M., Tomović, G. 10-Jul-2005, 19947 BEOU-KEGB, sub V. arvensis, rev. G. Tomović, 07-Sep-2013); Ušće, Žaračka planina, Gokčanica selo, UTM DP71, 700-800 m (leg. Stevanović, V., Niketić, M., Vukojičić, S., Tomović, G. 02-May-2004, det. G. Tomović, 18814 BEOU-KEGB, sub V. tricolor subsp. macedonica); Jastrebac, 8419 BEO, rev. G. Tomović, 23-Sep-2013); Veliki Jastrebac, Bela stena, UTM EP30, silikat (leg. Niketić, M. 14-May-2003, det. G. Tomović, BEO, sub V. macedonica); Sokolov kamen, UTM EP30, silikat (leg. Niketić, M. 13-May-2005, det. G. Tomović, BEO, sub V. macedonica); Kopaonik, granit (leg. Lakušić, D. 19-Apr-1986, det. D. Lakušić, 41249 BEOU, rev. G. Tomović, Apr2007); (8589 BEO, sub V. pancicii, rev. G. Tomović, Feb-2007); (leg. Mišić, V., Popović, M. 1952, det. V. Mišić, M. Popović, 41246 BEOU, rev. G. Tomović, Apr-2007); (leg. Pančić, J. May-1875, det. J. Pančić, 14598 BEOU (Herb. Panc.), sub V. macedonica); (leg. Pančić, J., det. J. Pančić, 3319 BEOU (Herb. Panc.), sub V. tricolor var. alpestris, rev. G. Tomović, Apr-2007); Bećirovac, UTM DN89 (leg. Pančić, J. Aug-1874, det. J. Pančić, 3230 BEOU (Herb. Panc.), sub V. gracilis, rev. G. Tomović, Apr-2007); Brus, UTM EP00, livade pored puta, 04-Jun-1985, det. G. Tomović, 41252 BEOU); Brzeće, UTM DN89, Exp. SE, 1300 m, stene (leg. Mišić, V., Popović, M. 26-May-1952, 41244 BEOU, sub V. saxatilis, rev. G. Tomović, Apr-2007); Jošanička banja, UTM DP70 (leg. Kuzmanović, N. 28-May-2012, det. G. Tomović, 38148 BEOU-KEGB, sub V. macedonica); Zablaće-Jarebica, UTM DP70 (leg. Rajevski, L., Borisavljević, Lj. 06-May-1954, det. G. Tomović, 41207 BEOU, sub V. macedonica); Jošanička banjaKlokovac, UTM DP70 (leg. Niketić, M., Tomović, G. 10-Jul-2005, det. G. Tomović, 19942 BEOU-KEGB, sub V. tricolor subsp. macedonica); Kadijevac, Hajdučki potok, uz, UTM DN89, 1600 m, livade, det. G. Tomović, 41247 BEOU); Kozje stene, UTM DN79, Exp. NE, 1550 m, krečnjak, rudine (leg. Lakušić, D., Niketić, M. 10-Aug-1986, det. M. Niketić, 41243 BEOU, sub V. macedonica); Krčmar, UTM DN89 (leg. Rudski, I. 09-Jul-1938, det. G. Tomović, 8483 BEO, sub V. saxatilis subsp. macedonica); Krmeljica, UTM DN79, 1100 m, serpentinit, Quercetum daleschampii (leg. Tomović, G. 29-May-2006, det. G. Tomović, 21195 BEOU-KEGB, sub V. tricolor subsp. macedonica); Metođe, Lisičje stene, UTM DN89, 1500 m, krečnjak, rudine (leg. Lakušić, D. 19-Jul-1988, det. D. Lakušić, 41248 BEOU, sub V. macedonica subsp. bosniaca, rev. G. Tomović, Apr-2007); Pančićev vrh, UTM DN89 (leg. Rudski, I. 08-Jul-1938, det. I. Rudski, 8485 BEO, sub V. saxatilis subsp. macedonica); Rudnica, put za, UTM DN78, 800 m, serpentinit, Querco-Carpinetum betuli (leg. Stevanović, V., Jovanović, S., Lakušić, D. 21Apr-1990, det. D. Lakušić, 41250 BEOU, sub V. macedonica); Suvo rudište, UTM DN89 (leg. Rudski, I. Oct-1938, det. I. Rudski, 8542 BEO, sub V. saxatilis, rev. G. Tomović, 23-Sep-2013); Treska, UTM DN88, serpentinit (leg. Niketić, M., Tomović, G., Novčić, R. 09-Jul-2005, det. G. Tomović, 19925 BEOUKEGB, sub V. tricolor subsp. macedonica); Vlajkovci, UTM DN99, 700 m, serpentinit, ArtemisioSilenetum armeriae (leg. Lakušić, D. 04-Jul-1989, det. D. Lakušić, 41245 BEOU, sub V. macedonica subsp. bosniaca, rev. G. Tomović, Apr-2007); (leg. Lakušić, D. 23-Apr-2005, det. G. Tomović, 19718 BEOU-KEGB, sub $V$. tricolor subsp. macedonica); ušće Male reke u Brzećku reku, UTM DN99, 800-900 m, serpentinit, kamenjari (leg. Niketić, M., Tomović, G. 14-May-2011, det. G. Tomović, 32118 BEOU-KEGB, sub V. macedonica); Vlajkovci-Brus, UTM DN99, EP00 (leg. Popović, J. 03-Aug-1999, 
det. J. Popović, 42036 BEOU, rev. G. Tomović, 07-Sep-2013); Pasjača, UTM EN47, EN57, Exp. SW, livade (leg. Nikolić, V. 13-May-1959, det. G. Tomović, 42797 BEO, sub V. arvensis, rev. G. Tomović, 23Sep-2013); kod groba, UTM EN47, EN57, Exp. SW, livade (leg. Nikolić, V. 14-Jul-1959, det. G. Tomović, 42796 BEO, sub V. arvensis); Prokuplje: Markovo gumno, UTM EN48 (leg. Jurišić, J. Ž. 01-Jun-1911, 8588 BEO, rev. G. Tomović, 23-Sep-2013); Stolovi (leg. Pančić, J. Jul-1877, det. G. Tomović, 3196 BEOU (Herb. Panc.), sub Viola sp.); (leg. Soška, Th. 04-Jun-1924, det. Th. Soška, 41238 BEOU, sub V. saxatilis, rev. G. Tomović, Apr-2007); (leg. Soška, Th. Jun-1926, det. Th. Soška, 8550 BEO, sub V. saxatilis, rev. G. Tomović, 23-Sep-2013); Meljanica reka - klisura, UTM DP72, serpentinit, kamenjari u vegetaciji Centaureo-Bromion fibrosi (leg. Zlatković, B. 07-Aug-2008, det. G. Tomović, 28465 BEOU-KEGB, sub V. macedonica); Studenica - dolina: manastir Studenica, UTM DP61 (leg. Soška, Th. 02-Jul-1932, det. Th. Soška, 41237 BEOU, sub V. saxatilis, rev. G. Tomović, Apr-2007); Savošnica reka, ušće, UTM DP61, livade (leg. Stevanović, V., Jovanović, S., Lakušić, D. 22Apr-1990, 204-90 BEOU-KEGB, sub V. saxatilis, rev. G. Tomović, 07-Sep-2013); Vidojevica: Jabučevo, na vrhu, UTM EN47, Exp. W, livade (leg. Nikolić, V. 15-Jul-1959, det. N. Diklić, 42178 BEO, sub V. tricolor subsp. tricolor, rev. G. Tomović, 23-Sep-2013); E Serbia Aleksinac, UTM EP62 (leg. Perić, 1929, det. G. Tomović, 41187 BEOU, sub V. macedonica); Gojmanovačka reka klisura, Labukovo, UTM EP71 (leg. Pančić, J., det. J. Pančić, 15012 BEOU (Herb. Panc.), sub V. tricolor var. saxatilis, rev. G. Tomović, 07-Sep-2013); Babička gora: Beli kamen-Ovčarevo, UTM EN87 (leg. Stojanović, D. 11-Jul-2006, det. G. Tomović, 28656 BEOU-KEGB, sub V. macedonica); Babušnica: Stol, UTM FN26 (leg. Soška, Th. 21-Jun-1923, det. G. Tomović, 41201, 41202 BEOU, sub V. macedonica); Devica: Koviljak, ispod vrha, UTM EP72, 1000 m, livade u vrtači (leg. Diklić, N. 24Jun-1957, det. N. Diklić, 43080 BEO, sub V. saxatilis subsp. macedonica); Oštra čuka, UTM EP72, $1200 \mathrm{~m}$, in pratis subalpinis (leg. Lindtner, V. 08-Jun-1956, det. G. Tomović, 43015 BEO, sub $V$. macedonica); sanatorijum, očni sanatorijum, UTM EP72 (leg. Nikolić, V., Diklić, N., Bogdanović, M. 19Jun-1972, det. G. Tomović, 43019 BEO, sub V. macedonica); Dimitrovgrad, UTM FN46 (leg. Simonović, D., det. G. Tomović, 41171 BEOU, sub V. macedonica); Greben planina: Poganovo, UTM FN35, in fauce (leg. Simonović, D. May-1923, 41170 BEOU, sub V. saxatilis, rev. G. Tomović, 07Sep-2013); Drenje, UTM FN35, livade (leg. Nikolić, V., Diklić, N., Rakin, M. 19-Jul-1964, det. N. Diklić, 43067 BEO, sub V. saxatilis subsp. macedonica); Niš: Kamenica, UTM EP70 (leg. Pančić, J. 1880, det. G. Tomović, 3202 BEOU (Herb. Panc.), sub Viola sp.); Ozren, UTM EP62, livade (leg. Diklić, N. Jun-1960, det. G. Tomović, 43076 BEO, sub V. macedonica); Lepterija, UTM EP62 (leg. Nikolić, V., Diklić, N., Bogdanović, M. 19-Jun-1972, det. G. Tomović, 42926 BEO, sub V. kitaibeliana, rev. G. Tomović, 23-Sep-2013); Leskovik, UTM EP62, kamenjari (leg. Pekić 24-Jun-1931, det. G. Tomović, 41186 BEOU, sub V. saxatilis, rev. G. Tomović, Apr-2007); (leg. Pančić, J. Jun-1870, det. G. Tomović, 3184 BEOU (Herb. Panc.), sub Viola sp.); sedlo, UTM EP62, pašnjaci (leg. Diklić, N. Jun-1960, det. G. Tomović, 43077 BEO, sub V. macedonica); Paraćin: Grza reka-klisura, Gornje jezero, UTM EP36, krečnjak, pored jezera (leg. Pejanović, v. 26-Apr-2001, 41192 BEOU, rev. G. Tomović, Apr-2007); motel, UTM EP36, krečnjak, livade (leg. Pejanović, V. 12-Jul-2001, 41191 BEOU, sub V. lutea, rev. G. Tomović, Apr-2007); (leg. Pejanović, V. 26-Apr-2001, 41189 BEOU, sub V. lutea, rev. G. Tomović, Apr2007); planinarski dom, UTM EP36, krečnjak, livade (leg. Pejanović, V. 25-Apr-2001, 41190 BEOU, sub V. arvensis, rev. G. Tomović, Apr-2007); Pirot, UTM FN28 (leg. Pančić, J. 1878, det. J. Pančić, 3312 BEOU (Herb. Panc.), sub V. tricolor var. arvensis); Jerma reka - klisura, Zvonačka banja, UTM FN35 (leg. Tomić, V. 05-Aug-1956, det. G. Tomović, VM-017 BEO, sub V. macedonica); Zvonačka banja, tvrđava Kale, UTM FN35, Exp. S (leg. Tomić, V. 13-Aug-1955, det. G. Tomović, VM-018, VM018 BEO, sub V. macedonica); Temštica reka - klisura, Mrtvački most, ispod, UTM FN39 (leg. Jovanović, S., Lakušić, D. 30-Jun-1991, det. G. Tomović, 453-91 BEOU-KEGB, sub V. macedonica); Rtanj, UTM EP64, EP74, Exp. E, 1300-1530 m, pašnjaci (leg. Diklić, N. 08-Jun-1965, det. G. Tomović, 43043, 43044 BEO, sub V. macedonica); Exp. S, 1000-1500 m, krečnjak, pašnjaci i kamenjari (leg. Nikolić, V., Diklić, N., Mladenović, S. 29-Jun-1980, det. G. Tomović, 43051 BEO, sub V. macedonica); (leg. 
Pančić, J. 1868, det. J. Pančić, 3324 BEOU (Herb. Panc.), sub V. tricolor var. bannatica (macedonica)); (leg. Pančić, J. 1869, det. J. Pančić, 15015 BEOU (Herb. Panc.), sub V. olympica; macedonica, rev. G. Tomović, 07-Sep-2013); (leg. Pančić, J., det. J. Pančić, 3248, 3249 BEOU (Herb. Panc.), sub V. macedonica); (leg. Soška, Th. Jun-1927, 41183 BEOU, sub V. saxatilis, rev. G. Tomović, Apr-2007); (leg. Soška, Th. Jun-1929, 41180, 41182, 41184 BEOU, sub V. saxatilis, rev. G. Tomović, Apr-2007); (leg. Soška, Th. 1922, det. Th. Soška, 8536 BEO, sub V. saxatilis, rev. G. Tomović, 23-Sep-2013); greben, UTM EP64, EP74, Exp. S, 1300 m, šibljak jorgovana (leg. Diklić, N. 09-Jun-1965, det. N. Diklić, 43093 BEO, sub V. saxatilis subsp. macedonica); kamenjari (leg. Nikolić, V., Diklić, N. 29-Jun-1971, det. N. Diklić, 43081 BEO, sub V. saxatilis subsp. macedonica); (leg. Lindtner, V. 11-Jun-1939, det. G. Tomović, VM-024 BEO, sub V. macedonica); Krstatac, UTM EP83, krečnjak (leg. Niketić, M., Tomović, G. 03-Jun2005, det. G. Tomović, BEO, sub V. macedonica); Tumba, greben, UTM EP74, 700-850 m, krečnjak, šibljaci i kamenjari (leg. Niketić, M., Tomović, G. 10-Jul-2009, 30189 BEOU-KEGB, sub V. macedonica); Ruj planina: Cvetkova livada, UTM FN24, livade (leg. Nikolić, V., Diklić, N. 14-Jul-1965, det. G. Tomović, 43041 BEO, sub V. macedonica); livade (leg. Nikolić, V., Diklić, N. 14-Jul-1965, det. N. Diklić, 43092 BEO, sub V. saxatilis subsp. macedonica); Tumba, Biljine bare, UTM FN24 (leg. Džukić, G. 21Sep-2007, det. G. Tomović, 39346 BEOU-KEGB, sub $V$. macedonica); Zvonce, UTM FN24, krečnjak, kamenjari (leg. Nikolić, V., Diklić, N. 18-Jun-1966, det. G. Tomović, 43037 BEO, sub V. macedonica); Rakita, Rakitska gora, UTM FN25 (leg. Nikolić, V., Diklić, N. 13-Jul-1965, det. N. Diklić, 43003 BEO, rev. G. Tomović, Feb-2007); Rakita, Vitkov del, UTM FN25, livade (leg. Nikolić, V., Diklić, N., Rakin, M. 22-Jul-1964, det. N. Diklić, 43068 BEO, sub V. saxatilis subsp. macedonica); Seličevica: Mala Tumba-Jezero, UTM EN78, silikat (leg. Niketić, M., Tomović, G. 11-Apr-2010, det. G. Tomović, BEO, sub V. macedonica); Stara planina, det. G. Tomović, 41185 BEOU); Rasovati kamen, UTM FP22, silikat (leg. Niketić, M. 10-Sep-2006, det. G. Tomović, BEO, sub V. macedonica); Ravno Bučje, UTM FP21 (leg. Pančić, J. 1875, det. J. Pančić, 3308 BEOU (Herb. Panc.), rev. G. Tomović, 07-Sep-2013); Ravno Bučje-Sveti Nikola, UTM FP21 (leg. Pančić, J. May-1875, det. J. Pančić, 3253 BEOU (Herb. Panc.), sub V. macedonica); Vetren, UTM FP22 (leg. Pančić, J. 1872, det. J. Pančić, 15008 BEOU (Herb. Panc.), sub V. tricolor var. alpestris, rev. G. Tomović, 07-Sep-2013); Vojvodin venac, UTM FP30, crveni peščar i konglomerat, planinski pašnjaci (leg. Mihailović, T. 24-Jul-1993, det. T. Mihailović, 41193 BEOU, rev. G. Tomović, Apr-2007); Suva planina, 1874, 41174 BEOU, rev. G. Tomović, Apr2007); (leg. Petrović, D. May, det. G. Tomović, 41173 BEOU, sub V. macedonica); (leg. Soška, Th., det. Th. Soška, 8513 BEO, sub V. saxatilis subsp. macedonica); Devojački grob-Trem, UTM EN98, krečnjak (leg. Tomović, G. 23-May-2005, det. G. Tomović, 19832 BEOU-KEGB, sub V. tricolor subsp. macedonica); UTM EN98, krečnjak (leg. Vukojičić, S., Tomović, G. 08-Jul-1997, det. G. Tomović, 6314 BEOU-KEGB, sub V. macedonica); 1400-1800 m, krečnjak (leg. Tomović, G., Zlatković, B. 31-May-2006, det. G. Tomović, 21215 BEOU-KEGB, sub V. tricolor subsp. macedonica); Gornja Studena, UTM EN88 (leg. Soška, Th. 10-Sep-1929, det. G. Tomović, 41176 BEOU, sub V. macedonica); (leg. Soška, Th. 10-Sep-1929, 41179 BEOU, sub V. saxatilis, rev. G. Tomović, Apr-2007); iznad, UTM EN88 (leg. Soška, Th. Jun-1910, 41177 BEOU, sub V. alpestris, rev. G. Tomović, Apr-2007); greben, UTM EN98, stene (leg. Nikolić, V., Diklić, N., Bogdanović; M. 18-Jun-1973, det. G. Tomović, 43026, 43027 BEO, sub V. macedonica); Mosor, UTM EN88, krečnjak, stene (leg. Stevanović, V., Lakušić, D. 07-Apr-1990, 25-91 BEOU-KEGB, sub V. saxatilis, rev. G. Tomović, 07-Sep-2013); Mosor-Sokolov kamen, UTM EN88, EN98, krečnjak (leg. Vukojičić, S., Tomović, G. 06-Jul-1997, det. G. Tomović, 6524 BEOU-KEGB, sub V. macedonica); Smrdan, UTM FN07, FN08, livade (leg. Janković, M. 08-Jun-1947, det. G. Tomović, 41175 BEOU, sub V. macedonica); Svrljiške planine: Goli vrh, UTM FN09, Exp. S (leg. Nikolić, V., Diklić, N. 30-Jun-1963, det. N. Diklić, 43070 BEO, sub V. saxatilis subsp. macedonica); Pleš, UTM EP90 (leg. Pančić, J. 1872, det. J. Pančić, 3317 BEOU (Herb. Panc.), sub V. tricolor var., rev. G. Tomović, 07-Sep-2013); (leg. Pančić, J., det. J. Pančić, 3247 BEOU (Herb. Panc.), sub V. macedonica); Tupižnica, UTM EP94 (leg. Soška, Th. Jun-1924, 41188 BEOU, sub V. saxatilis, rev. G. Tomović, Apr2007); Vidlič: Basara, UTM FN38 (leg. Lindtner, V. 14-Jun-1956, det. G. Tomović, VM-021 BEO, sub 
V. macedonica); Basarski kamen, na vrhu, UTM FN38, $1371 \mathrm{~m}$, krečnjak, kamenjari (leg. Nikolić, $V$. 15-Jun-1976, det. G. Tomović, 43054 BEO, sub V. macedonica); Vlaška planina: Golema glava, UTM FN25, kamenjari (leg. Nikolić, V., Diklić, N. 19-Jul-1965, det. G. Tomović, 43039, 43040 BEO, sub V. macedonica); Zvonačka banja, UTM FN35, 1300 m, stene (leg. Nikolić, V., Diklić, N., Rakin, M. 21-Jul1964, det. N. Diklić, 43071 BEO, sub V. saxatilis subsp. macedonica); Quercetum cerris (leg. Nikolić, V., Diklić, N., Rakin, M. 19-Jul-1964, det. N. Diklić, 43069 BEO, sub V. saxatilis subsp. macedonica); severno od banje, UTM FN35, hrastova šuma (leg. Nikolić, V., Diklić, N., Rakin, M. 15-Jul-1965, det. G. Tomović, 43038 BEO, sub V. macedonica); livade (leg. Nikolić, V., Diklić, N., Rakin, M. 15-Jul-1965, det. G. Tomović, 43046 BEO, sub V. macedonica); Trnski Odorovci, put za selo, UTM FN35 (leg. Nikolić, V., Diklić, N. 19-Jun-1969, det. G. Tomović, 43036 BEO, sub V. macedonica); SW Serbia Giljeva, 1300 m, krečnjak, pašnjaci i kamenjari (leg. Jovanović, S., 23-Jun-1996, det. G. Tomović, 2641-96 BEOUKEGB, sub V. macedonica); Dugi dol, Brežđe, UTM DN18, 1295 m, krečnjak, Brometum (leg. Đorđević, V. 26-Jun-2014, det. G. Tomović, 40643 BEOU-KEGB, sub V. tricolor subsp. macedonica); Krajinovići, Čelo brdo, UTM DN08, 1000 m, serpentinit (leg. Stevanović, V., Niketić, M., Vukojičić, S., Tomović, G. 28-Apr-2006, det. G. Tomović, 20728 BEOU-KEGB, sub V. tricolor subsp. macedonica); Trijebine, Prijevorac brdo, UTM DN18, 1250 m, serpentinit (leg. Stevanović, V., Niketić, M., Vukojičić, S., Tomović, G. 28-Apr-2006, det. G. Tomović, 20731 BEOU-KEGB, sub V. tricolor subsp. macedonica); Trijebinska reka - klisura, UTM DN18, 1100 m, krečnjak (leg. Stevanović, V., Niketić, M., Vukojičić, S., Tomović, G. 28-Apr-2006, det. G. Tomović, 20765 BEOU-KEGB, sub V. tricolor subsp. macedonica); Golija: Dajići, UTM DP40 (leg. Pančić, J. 1875, det. G. Tomović, 3188 BEOU (Herb. Panc.), sub Viola sp.); Javor: Jankov vrh, UTM DP21, 1492 m, krečnjak, Festuco-Brometea (leg. Đorđević, v. 29-Jun2014, det. G. Tomović, 40642 BEOU-KEGB, sub V. tricolor subsp. macedonica); Nova Varoš: Čajo (leg. Ilić, S. 10-May-1929, det. S. Ilić, 41234 BEOU, sub V. alpestris, rev. G. Tomović, Apr-2007); Novi Pazar: Novi Pazar - Ribariće, UTM DN56, DN57 (leg. Soška, Th. Jun-1914, det. Th. Soška, 41231 BEOU, sub $V$. alpestris, rev. G. Tomović, Apr-2007); Pešter: Duga poljana, UTM DN17, livade (leg. Nikolić, V., Mladenović, S. 15-Jul-1981, det. G. Tomović, 43053 BEO, sub V. macedonica); Duga Poljana - Kamešnica, UTM DN38, krečnjak, livade pored puta (leg. Niketić, M., Tomović, G., Buzurović, U. 11-May-2014, det. G. Tomović, 40641 BEOU-KEGB, sub V. tricolor subsp. macedonica); Karajukića bunari, skretanje za Suvi do, UTM DN26, krečnjak, livade pored puta (leg. Niketić, M., Tomović, G., Buzurović, U. 11-May-2014, det. G. Tomović, 40638 BEOU-KEGB, sub V. tricolor subsp. macedonica); Sjenica, okolina, serpentinit, livade i kamenjari (leg. Jovanović, S. 23-Jun-1996, det. G. Tomović, 2727-96 BEOU-KEGB, sub V. macedonica); Stup selo, Vrelo reka - klisura, UTM DN29, 1200 m, krečnjak (leg. Stevanović, V., Niketić, M., Vukojičić, S., Tomović, G. 27-Apr-2006, det. G. Tomović, 20683 BEOU-KEGB, sub V. tricolor subsp. macedonica); Prijepolje: Jabuka, UTM CP70, 1100 m, krečnjak, kamenjari (leg. Stevanović, V., Niketić, M., Vukojičić, S., Tomović, G. 29-Apr-2006, det. G. Tomović, 20873 BEOU-KEGB, sub $V$. tricolor subsp. macedonica); Rogozna: Grižani HanKaraula, UTM DN76 (leg. Soška, Th. Jun-1914, det. Th. Soška, 41240 BEOU, rev. G. Tomović, Apr2007); Stolovi, Šanac, UTM DN76, 1300 m, andezit, pašnjaci i kamenjari (leg. Niketić, M., Tomović, G. 08-Jul-2010, det. G. Tomović, 30711 BEOU-KEGB, sub V. macedonica); (leg. Niketić, M., Tomović, G. 08Jul-2010, det. G. Tomović, BEO, sub V. macedonica); Tutin: Koštam polje, UTM DN47, livade (leg. Košanin, N. 21-Jun-1926, det. G. Tomović, 41229 BEOU, sub V. macedonica); Zlatar: Mokra poljanaGolo brdo, UTM DP00, 1200-1500 m, livade (leg. Nikolić, V., Diklić, N. 16-Jul-1971, det. N. Diklić, 43004 BEO, sub V. dacica, rev. G. Tomović, Feb-2007); 1400-1600 m, krečnjak, livade (leg. Nikolić, V., Diklić, N. 21-Jun-1970, det. G. Tomović, 43033 BEO, sub V. macedonica); 1600 m, krečnjak, livade (leg. Nikolić, V., Diklić, N. 21-Jun-1970, det. G. Tomović, 43034 BEO, sub V. macedonica); Velika Kršava, UTM DP00, Exp. SE, 1616 m, krečnjak, Festuco-Brometea (leg. Đorđević, v. 23-May-2014, det. G. Tomović, 40646 BEOU-KEGB, sub $V$. tricolor subsp. macedonica); S Serbia Bujanovac: Binačka Morava, Končulj klisura, UTM EN50, EM59, kamenjari, čistine u šumi sladuna i cera (leg. Nikolić, V., Diklić, N., 16-Jun-1975, det. N. Diklić, 43176 BEO, sub V. tricolor subsp. tricolor, rev. G. Tomović, 23- 
Sep-2013); Končulj klisura, Lučane okolina, UTM EN50, EM59, hrastova šuma (leg. Nikolić, v. 06Jun-1967, det. V. Nikolić, 43008 BEO, sub V. macedonica); Levosoje, UTM EM69, antimor, stene (leg. Zlatković, B. 28-Apr-2012, det. G. Tomović, 34946 BEOU-KEGB, sub V. macedonica); Lopardince, iznad sela, UTM EN60, Exp. W, kamenjari (leg. Nikolić, V., Diklić, N. 23-Apr-1965, det. N. Diklić, 42833 BEO, sub V. dacica, rev. G. Tomović, 23-Sep-2013); Kukavica, UTM EN73 (leg. Ostojić, D. 15Nov-1996, det. G. Tomović, 2990 BEOU-KEGB, sub V. macedonica); Lebane, UTM EN55 (leg. Pančić, J. 21-Jul-1885, det. J. Pančić, 15000 BEOU (Herb. Panc.), sub V. gracilis, rev. G. Tomović, 07Sep-2013); Pljačkavica, UTM EN71, 41169 BEOU, rev. G. Tomović, 07-Sep-2013); (leg. llić, Đ. Apr1911, det. Đ. Ilić, 8492 BEO, sub V. macedonica); (leg. llić, Đ. 1903, det. G. Tomović, 41957 BEOU, sub V. macedonica); (leg. llić, Đ. 1903, 41958 BEOU, rev. G. Tomović, 07-Sep-2013); (leg. Ničić, Đ. 12-Jul1886, det. G. Tomović, 41956 BEOU, sub V. macedonica); Preševo: brdo iznad sela Miratovac, kod punkta, UTM EM57, silikati (leg. Tomović, G., Zlatković, B. 01-Jun-2014, det. G. Tomović, 40740 BEOU-KEGB, sub V. tricolor subsp. macedonica); Radan: Petrova gora, Šopot, UTM EN46, Exp. E, livade (leg. Nikolić, V. 21-Jul-1959, det. G. Tomović, 43031 BEO, sub V. macedonica); Sokolov vis, Veliki krš, UTM EN35, silikat, stene (leg. Niketić, M., Tomović, G. 01-May-2008, det. G. Tomović, 26913 BEOU-KEGB, sub V. macedonica); vrh, UTM EN35, silikat, stene (leg. Niketić, M., Tomović, G. 01-May2008, det. G. Tomović, 26873 BEOU-KEGB, sub V. macedonica); Velika Loparda, UTM EN46, bukova šuma (leg. Nikolić, V. 23-Jun-1960, det. G. Tomović, 43007 BEO, sub V. macedonica); Rujan: prevoj - Svinjište, UTM EM68, c. $700 \mathrm{~m}$, silikati, pored puta (leg. Tomović, G., Zlatković, B. 01-Jun2014, det. G. Tomović, 40744 BEOU-KEGB, sub V. tricolor subsp. macedonica); Sijarinska banja: Sijarina selo, Tupalski vis, UTM EN54 (leg. Nikolić, V. 24-Jun-1965, det. G. Tomović, 43010 BEO, sub V. macedonica); Vranje, det. G. Tomović, 41167 BEOU); Gnjilanska ulica, UTM EN71, gažene ruderalne površine, 09-May-2000, 41166 BEOU, rev. G. Tomović, Apr-2007); Markovo kale, UTM EN71 (leg. Pančić, J. 1878, det. J. Pančić, 3254 BEOU (Herb. Panc.), sub V. macedonica (tricolor)); Oblik, UTM EN72 (leg. Niketić, M., Tomović, G., Zlatković, B. 04-May-2002, det. G. Tomović, 15519 BEOU-KEGB, sub V. macedonica); vrh, UTM EN72, 1300 m, kamenjari (leg. Diklić, N. 01-Jun-1960, det. N. Diklić, 43072 BEO, sub V. saxatilis subsp. macedonica); Vrtogoš, Karpina, UTM EN60 (leg. Pančić, J. May-1885, det. G. Tomović, 3209 BEOU (Herb. Panc.), sub Viola sp.); (leg. Pančić, J. May1886, det. G. Tomović, 3210 BEOU (Herb. Panc.), sub Viola sp.); SE Serbia Besna kobila: Kriva Feja, UTM EN90, $1180 \mathrm{~m}$, silikat, osuline pored puta (leg. Lakušić, D., Vukojičić, S., Kuzmanović, N., 17Jul-2012, det. G. Tomović, 37232 BEOU-KEGB, sub V. macedonica); planinarski dom, UTM FN00, 1500-1600 m, livade (leg. Nikolić, V., Diklić, N. 22-Jul-1967, det. N. Diklić, 43086 BEO, sub V. saxatilis subsp. macedonica); Bosilegrad: Karamanica, rudnik - Gornje Tlamino, UTM FM09, $1626 \mathrm{~m}$, silikat, livade (leg. Tomović, G., Zlatković, B., Tomović, Lj. 22-Jun-2014, det. G. Tomović, 40767 BEOUKEGB, sub V. tricolor subsp. macedonica); Valozi, UTM FN01, 1700-1800 m, silikat (leg. Niketić, M., Tomović, G., Zlatković, B., Anačkov, G. 15-Aug-2006, det. G. Tomović, BEO, sub V. macedonica); Čemernik, Exp. NE, 1300 m, livade (leg. Diklić, N. 26-Jul-1966, det. G. Tomović, 43042 BEO, sub V. macedonica); Grdelička klisura: Vladičin Han, Kržince, UTM EN83, pored puta i žbunja (leg. Jurišić, J. Ž. 21-May-1911, 8587 BEO, rev. G. Tomović, 23-Sep-2013); Kozjak: manastir Prohor Pčinjski, okolina manastira, UTM EM78 (leg. Nikolić, V., Diklić, N. 15-May-1975, det. G. Tomović, 43020, 43021 BEO, sub V. macedonica); Ostrozub, UTM FN04, 17-Jul-1950, 41137 BEOU, rev. G. Tomović, Apr-2007); Pčinja - klisura: manastir Prohor Pčinjski, na putu prema, UTM EM78 (leg. Jovanović, S., Vukojičić, S., Pavić, S. 04-Jun-1995, det. G. Tomović, 1599-95 BEOU-KEGB, sub V. tricolor subsp. macedonica); Trgovište-Radovnica, UTM EM99, FM09, kamenjari (leg. Nikolić, V., Diklić, N. 11-May-1973, det. G. Tomović, 43029, 43030 BEO, sub V. macedonica); Vardenik, UTM FN01, FN02 (leg. Černjavski, P., Pavlović, Z., Mišić, V. 20-May-1947, det. G. Tomović, 41151 BEOU, sub V. macedonica); Vlasina, $1250 \mathrm{~m}$, oranice, 09-Jul-1950, 41145 BEOU, rev. G. Tomović, Apr-2007); livade (leg. Milovanović, D. 30-Jun-1947, det. G. Tomović, 41152 BEOU, sub V. macedonica); (leg. Milovanović, D. 30-Jun-1947, det. G. Tomović, 41143 BEOU, sub V. macedonica); (leg. Ničić, Đ. 19-Jul- 
1886, 41159 BEOU, sub V. macedonica, rev. G. Tomović, Apr-2007); (Jun-1907, 41157 BEOU, sub V. tricolor saxatilis, rev. G. Tomović, Apr-2007); (Jul-1910, det. W. Becker, 41158 BEOU, rev. G. Tomović, Apr-2007); (leg. Černjavski, P. 08-Aug-1930, det. P. Černjavski, 8500 BEO, sub V. saxatilis subsp. macedonica); (leg. Ilić, Đ. Jun-1910, det. Đ. Ilić, 8523 BEO, sub V. macedonica); (leg. Milovanović, D. 15-Jul-1948, det. G. Tomović, 41153 BEOU, sub V. macedonica ?); (leg. Milovanović, D. 22-Jul-1948, det. G. Tomović, 41154 BEOU, sub V. macedonica ?); (leg. Mišić, V., Janković, M. 09-Oct-1949, det. G. Tomović, 41148 BEOU, sub V. macedonica); (leg. Nikolić, V., Diklić, N., Bogdanović, M. 25-Jul-1972, det. G. Tomović, 43022 BEO, sub V. macedonica); (leg. Pančić, J. 1880, det. J. Pančić, 3314 BEOU (Herb. Panc.), rev. G. Tomović, 07-Sep-2013); (leg. Pančić, J. 1881, det. G. Tomović, 3208 BEOU (Herb. Panc.), sub Viola sp.); (leg. Petrov, N. 23-May-1948, det. G. Tomović, 41144 BEOU, sub V. macedonica); (leg. Petrov, N. 23-May-1948, det. G. Tomović, 41156 BEOU, sub V. macedonica ?); (leg. Petrov, N., Mišić, V. 23-May-1948, det. G. Tomović, 41164 BEOU, sub V. macedonica); (leg. Soška, Th. Jun-1910, det. Th. Soška, 8511, 8512 BEO, sub V. saxatilis subsp. macedonica); (leg. Soška, Th. Jun-1910, det. Th. Soška, 8525, 8526 BEO, sub V. saxatilis subsp. macedonica); (leg. Soška, Th. Jul-1910, 41160 BEOU, sub V. alpestris, rev. G. Tomović, Apr-2007); (leg. Soška, Th. Jul-1910, det. Th. Soška, 41138 BEOU, rev. G. Tomović, Apr-2007); Crkvena mala, ispod, UTM FN03 (leg. Černjavski, P., Pavlović, J., Mišić, V. 21-May-1947, det. G. Tomović, 42009 BEOU, sub V. macedonica); kod, UTM FN03, bukova šuma (leg. Černjavski, P. 13-Aug-1930, 8571 BEO, rev. G. Tomović, 23-Sep-2013); usek, UTM FN03 (leg. Černjavski, P. 13-Aug-1930, det. P. Černjavski, 8522 BEO, sub V. saxatilis subsp. macedonica); usek na putu, UTM FN03 (leg. Černjavski, P. 08-Aug-1930, det. P. Černjavski, 8519 BEO, sub V. saxatilis subsp. macedonica); Lisinska reka - klisura, UTM FN11 (leg. Jovanović, S., Niketić, M. 30-Aug-1991, det. G. Tomović, 1864-91 BEOU-KEGB, sub V. macedonica); Mali vrh, UTM FN13, Exp. S, SW, 1600 m, stene (leg. Jovanović, S., Niketić, M. 29-Aug-1991, 1780-91 BEOU-KEGB, sub V. dacica, rev. G. Tomović, 07-Sep-2013); Okruglica, iznad, UTM FN02, bukova šuma (leg. Černjavski, P., Pavlović, Z., Mišić, V. 25-May-1947, det. G. Tomović, 41140 BEOU, sub V. macedonica); Okruglica-Crkvena mala, kraj puta, UTM FN02, FN03, utrine (leg. Černjavski, P., Pavlović, Z., Mišić, V. 21-May-1947, det. G. Tomović, 41155 BEOU, sub V. macedonica); Pandžin grob, UTM FN02, bukov šibljak (leg. Černjavski, P., Pavlović, Z., Mišić, V. 25-May-1947, det. G. Tomović, 41149 BEOU, sub V. macedonica); Plana, Vlasina reka, UTM FN04, FN14, tresave (leg. Černjavski, P., Pavlović, Z., Mišić, V. 28-May-1947, det. G. Tomović, 41147 BEOU, sub V. macedonica); vrh, UTM FN04, FN14, kamenjari (leg. Černjavski, P. 16-Aug-1930, det. P. Černjavski, 8493 BEO, sub V. saxatilis subsp. macedonica); Vrtop, UTM FN13, Festuco-Agrostietum nardetosum (leg. Jovanović, S., Niketić, M. 29-Aug-1991, 1717-91 BEOU-KEGB, sub V. dacica, rev. G. Tomović, 07-Sep-2013); Stratorija, UTM FN12, Jul-1910, 41161 BEOU, rev. G. Tomović, Apr-2007); (leg. Košanin, N. 07-Jun-1907, det. N. Košanin, 41139 BEOU, sub V. alpestris, rev. G. Tomović, Apr-2007); (leg. Košanin, N. Jul-1910, det. N. Košanin, 41163 BEOU, sub V. lutea, rev. G. Tomović, Apr-2007); (leg. Soška, Th. Jul-1910, det. G. Tomović, 41162 BEOU, sub V. macedonica); Veliki most, UTM FN12 (leg. Mišić, V., Janković, M. 08-Oct-1947, det. G. Tomović, 41146 BEOU, sub V. macedonica); Vlasina rid, UTM FN03, 1000 m, silikat, livade (leg. Lakušić, D., Tomović, G., Vukojičić, S., Kabaš, E., Batansjki, V., Duraki, Š. 19-Jun-2010, det. G. Tomović, 30398 BEOU-KEGB, sub V. macedonica); Vlasinsko jezero, 1200 m, livade (leg. Diklić, N. 23-Jul-1966, det. G. Tomović, 43049 BEO, sub V. macedonica); livade (leg. Nikolić, V., Diklić, N. 13-17-Jun-1967, det. G. Tomović, 43050 BEO, sub V. macedonica); livade (leg. Nikolić, V., Diklić, N. 13-17-Jun-1967, det. G. Tomović, 43032 BEO, sub V. macedonica); (leg. Nikolić, V., Diklić, N. 13-17-Jun-1967, det. N. Diklić, 43088 BEO, sub V. saxatilis subsp. macedonica); Dugi Del poluostrvo, UTM FN12, zakorovljena poljoprivredna površina i obale jezera (leg. Jovanović, S., Niketić, M. 31-Aug-1991, 1988-91 BEOU-KEGB, sub V. dacica, rev. G. Tomović, 07-Sep-2013); Stankovci-Dugi Del poluostrvo, UTM FN12, Exp. SE, vlažna depresija (leg. Jovanović, S., Niketić, M. 31-Aug-1991, 2003-91 BEOU-KEGB, sub V. dacica, rev. G. Tomović, 07-Sep2013); Vodojaža, UTM FN03, utrine u žbunju (leg. Černjavski, P., Pavlović, Z., Mišić, V. 24-May-1947, det. G. Tomović, 41142 BEOU, sub V. macedonica); iznad, UTM FN03, pored potoka (leg. Černjavski, 
P., Pavlović, J., Mišić, V. 24-May-1947, det. G. Tomović, 42014 BEOU, sub V. macedonica); (leg. Černjavski, P., Pavlović, Z., Mišić, V. 24-May-1947, det. G. Tomović, 41165 BEOU, sub V. macedonica); utrine (leg. Černjavski, P., Pavlović, Z., Mišić, V. 22-May-1947, det. G. Tomović, 41150 BEOU, sub V. macedonica); kraj puta, UTM FN03 (leg. Černjavski, P., Pavlović, Z., Mišić, V. 24-May-1947, det. G. Tomović, 41141 BEOU, sub V. macedonica); Vlasotince: Jastrebac-Donja Lopušnja, UTM EN95, pored puta (leg. Niketić, M., Tomović, G. 30-Apr-2008, det. G. Tomović, 26851 BEOU-KEGB, sub V. macedonica); Vranje: Vranjska banja, UTM EN81 (leg. Pančić, J. 1878, det. G. Tomović, 3197 BEOU (Herb. Panc.), sub Viola sp.); Kosovo Ibarska dolina: Kosovska Mitrovica, UTM DN94, nasute deponije, nitrifikovane obale reka, železnički i putni nasipi (leg. Milinčić, D., 01-Jun-1996, det. D. Milinčić, 41213 BEOU, sub V. arvensis var. arvensis, rev. G. Tomović, Apr-2007); Kopaonik: Oštro koplje, Kačandol-Bele stene, UTM EN06 (leg. Soška, Th. 30-Jun-1914, det. Th. Soška, 41241 BEOU, sub V. alpestris, rev. G. Tomović, Apr-2007); Rogozna: Stolovi, Čapljenac, UTM DN76, serpentinit (leg. Niketić, M., Tomović, G. 08-Jul-2010, det. G. Tomović, BEO, sub V. macedonica); $1318 \mathrm{~m}$, serpentinit, pašnjaci i kamenjari (leg. Niketić, M., Tomović, G. 08-Jul-2010, 30716 BEOU-KEGB, sub $V$. macedonica); Šar-planina: Kodža Balkan, Tumba, UTM DM87, Exp. SE, 1400-1500 m, livade (leg. Nikolić, V., Diklić, N. 18-Jul-1976, det. G. Tomović, 42995 BEO, sub V. macedonica); (leg. Nikolić, V., Diklić, N. 18-Jul-1976, det. G. Tomović, 42994 BEO, sub V. macedonica, rev. G. Tomović, 23-Sep-2013); Ošljak, Gornje selo, UTM DM97 (leg. Košanin, N. 29-May-1922, det. N. Košanin, 41134, 41135 BEOU, sub V. alpestris, rev. G. Tomović, Apr-2007); Popovo prase, UTM DM97, Exp. SE, 1700 m, livade (leg. Nikolić, V., Diklić, N. 23-Jul-1975, det. G. Tomović, 42992 BEO, sub V. macedonica); livade (leg. Nikolić, V., Diklić, N., Mladenović, S. 23-Jul-1976, det. G. Tomović, 42991 BEO, sub V. macedonica); Sevce, UTM DM97 (leg. Nikolić, V., Diklić, N., Mladenović, S. 23-Jul-1976, det. G. Tomović, 42996 BEO, sub V. macedonica); Prevalac, Svinjarski potok, oko karaule, UTM DM96 (leg. Rudski, I. 16-Jul1930, det. I. Rudski, 41132 BEOU, sub V. saxatilis, rev. G. Tomović, Apr-2007); Uroševac: Nerodimka, oko razvođa, UTM EM08 (leg. Jurišić, Ž. J. 03-May-1914, det. Ž. J. Jurišić, 8541 BEO, sub V. lutea, rev. G. Tomović, 23-Sep-2013); Metohija Mokra gora (leg. Rudski, I., 16-Jul-1932, det. G. Tomović, VM-025 BEO, sub V. macedonica); Radavac, UTM DN44, bukova šuma (leg. Janković, M. 02-May-1960, det. G. Tomović, 41131 BEOU, sub V. macedonica); Prokletije: Dečanska planina, UTM DN31, bukovo-molikova šuma (leg. Černjavski, P., Rudski, I., Lindtner, V. 15-Jul-1933, det. P. Černjavski, 8484 BEO, sub V. saxatilis subsp. macedonica); (leg. Lindtner, V. 15-Jul-1933, det. G. Tomović, VM-020 BEO, sub V. macedonica); Kožnjar, Baba Loć-Maja Rops, UTM DN21 (leg. Janković, M. 13-16. Jul-1964, det. M. Janković, 41296 BEOU, sub V. elegantula, rev. G. Tomović, 21Dec-2012); Krstac-Hajla, UTM DN23 (leg. Gošović, S. Aug-1922, det. S. Gošović, 41124 BEOU, sub V. alpestris, rev. G. Tomović, Apr-2007); Kurvala, Ločanska planina, UTM DN31, molikova šuma (leg. Černjavski, P., Rudski, I., Lindtner, V. 15-Jul-1933, det. P. Černjavski, 8469 BEO, sub V. elegantula var. latisepala, rev. G. Tomović, Feb-2007); Paklen, UTM DN32, krečnjak, in saxosis (leg. Soška, Th. 18-Jun-1923, det. Th. Soška, 41128 BEOU, sub V. alpestris, rev. G. Tomović, Apr-2007); Prilepska planina, UTM DN21 (leg. Janković, M. 1960, det. M. Janković, 41127 BEOU, sub V. arvensis, rev. G. Tomović, Apr-2007); (leg. Janković, M. 20-Jun-1957, det. G. Tomović, 41129 BEOU, sub V. macedonica); Raški do-Savine vode-Amšor-Žljeb-Kovraga, UTM DN21, DN22 (leg. Janković, M. 28-May 07-Jun-1960, det. G. Tomović, 41130 BEOU, sub V. macedonica); Žljeb (Maja Rusolija), Stubica, UTM DN33, 1500 m (leg. Rudski, I. 24-Jun-1932, det. G. Tomović, VM-016 BEO, sub V. macedonica); Šar-planina: Bistrica, Ploča, UTM DM96 (leg. Soška, Th. 20-Jun-1924, det. Th. Soška, 41136 BEOU, sub V. alpestris, rev. G. Tomović, Apr-2007); Brod-Dragaš, UTM DM74, DM75, Exp. NW (leg. Nikolić, V., Diklić, N. 11-Jul-1968, det. N. Diklić, 43089 BEO, sub V. saxatilis subsp. macedonica); Gradski kamen, S. brdo, UTM DM74 (leg. Stevanović, v. 10-Jun-1986, det. G. Tomović, 162-86 BEOU-KEGB, sub $V$. tricolor subsp. macedonica); Lokvice-planinarski dom, UTM DM86, 6001100 m (leg. Niketić, M., Tomović, G., Duraki, Š. 07-Jul-2007, det. G. Tomović, 24467 BEOU-KEGB, sub V. macedonica); Nebregošte, UTM DM86 (leg. Rudski, I. 02-Jul-1930, det. I. Rudski, 41133 BEOU, sub V. saxatilis, rev. G. Tomović, Apr-2007). 


\section{Analysis of specimens and taxa}

The examination of Herbaria BEO and BEOU has shown presence of 820 herbarium sheets including specimens of taxa from section $V$. sect. Viola. Of these, 427 sheets are deposited in collection BEO and 393 sheets

Table 1. - Number of specimens of Viola species/subspecies deposited in BEO and BEOU.

\begin{tabular}{|c|c|c|c|c|c|}
\hline Taxon & BEO & $\begin{array}{c}\text { BEOU } \\
\text { (General) }\end{array}$ & $\begin{array}{c}\text { BEOU } \\
\text { (Herb Panc) }\end{array}$ & $\begin{array}{c}\text { BEOU } \\
\text { (KEGB) }\end{array}$ & Total \\
\hline \multicolumn{6}{|l|}{$V$. sect. Viola } \\
\hline$V$. alba & 85 & 16 & 3 & 10 & 114 \\
\hline V. ambigua & 7 & 27 & 0 & 4 & 38 \\
\hline$V$. canina & 11 & 14 & 6 & 10 & 41 \\
\hline V. elatior & 3 & 0 & 0 & 0 & 3 \\
\hline$V$. hirta & 38 & 13 & 8 & 12 & 71 \\
\hline$V$. jordanii & 29 & 9 & 3 & 9 & 50 \\
\hline V. mirabilis & 6 & 3 & 3 & 4 & 16 \\
\hline V. obliqua & 1 & 1 & 0 & 4 & 6 \\
\hline$V$. odorata & 54 & 12 & 2 & 12 & 80 \\
\hline$V \cdot$ pumila & 0 & 0 & 1 & 0 & 1 \\
\hline V. reichenbachiana & 69 & 34 & 2 & 12 & 117 \\
\hline$V$. riviniana & 80 & 70 & 16 & 27 & 193 \\
\hline$V$. rupestris & 1 & 4 & 1 & 5 & 11 \\
\hline$V$. stagnina & 5 & 1 & 2 & 0 & 8 \\
\hline \multirow[t]{2}{*}{$V$. suavis } & 38 & 22 & 1 & 10 & 71 \\
\hline & 427 & 226 & 48 & 119 & 820 \\
\hline \multicolumn{6}{|l|}{ V. sect. Dischidium. } \\
\hline V. biflora & 9 & 14 & 3 & 3 & 29 \\
\hline \multicolumn{6}{|l|}{ V. sect. Melanium } \\
\hline$V$. aetolica & 12 & 8 & 0 & 3 & 23 \\
\hline$V$. arvensis & 49 & 28 & 8 & 17 & 102 \\
\hline$V$. calcarata subsp. zoysii & 16 & 3 & 0 & 1 & 20 \\
\hline$V$. dacica & 46 & 33 & 23 & 22 & 124 \\
\hline V. dukadjinica & 0 & 0 & 0 & 3 & 3 \\
\hline V. elegantula & 53 & 40 & 1 & 31 & 125 \\
\hline V. grisebachiana & 17 & 9 & 4 & 3 & 33 \\
\hline V. hymettia & 3 & 2 & 1 & 4 & 10 \\
\hline V. kitaibeliana & 42 & 12 & 11 & 34 & 99 \\
\hline$V$. orphanidis & 24 & 11 & 0 & 7 & 42 \\
\hline \multirow[t]{2}{*}{ V. tricolor } & 204 & 133 & 51 & 118 & 506 \\
\hline & 466 & 279 & 99 & 243 & 1087 \\
\hline Total & 902 & 519 & 150 & 365 & 1936 \\
\hline
\end{tabular}


in collection BEOU. The survey and analysis of herbarium material collected within the territory of Serbia have shown presence of 15 species from this section. Two species listed in edition "Flora of SR Serbia" (Diklić 1972) was not recorded in Serbia: V. uliginosa Besser and V. pyrenaica Ramond. The least number of herbarium data was recorded for species $V$. pumila (a single record) and $V$. elatior (three records). On the basis of the review of herbarium material, species $V$. stagnina, $V$. ambigua, $V$. rupestris and $V$. mirabilis may be treated as rare species in Serbia, as they were recorded in a relatively low number of UTM squares (8-16).

The examination has also shown presence of 29 herbarium sheets including specimens of species $V$. biflora $\mathrm{L}$. from section $V$. sect. Dischidium. This includes 9 sheets deposited in collection BEO and 20 sheets in collection of BEOU.

Herbaria BEO and BEOU include 1087 herbarium specimens of taxa belonging to section $V$. sect. Melanium. This includes 466 sheets deposited in the collection BEO and 621 sheets in collection BEOU. The survey and analysis of herbarium material from section $V$. sect. Melanium collected in territory of Serbia have shown presence of 11 taxa. Species $V$. speciosa Pant. listed in the edition "Flora of SR Serbia" (Diklić 1972) is, according to more recent monographs (Erben 1985), considered a synonym for species $V$. elegantula Schott. The smallest number of herbarium exsiccates (only three) was collected for species $V$. dukadjinica, while the greatest number (506) was collected for $V$. tricolor $\mathrm{s} .1$.

Detailed presentation of number of exsiccates in collections BEO and BEOU, sorted by sections and species/subspecies is presented in Table 1.

\section{DISCUSSION AND CONCLUSIONS}

According to Mereda et al. (2008), the genus Viola is taxonomically demanding to resolve because (a) high phenotypic plasticity, (b) frequent interspecific hybridizations, (c) scarcity of reliable diagnostic morphological characters, (d) assumed past or even recent reticulate evolution.

In species of the $V$. sect. Viola the intrapopulation and interpopulation variability of morphological characters is high, representing a big challenge for identification of certain species, particularly in case of older herbarium specimens. After the revision of herbarium material it was concluded that more than $1 / 3$ of exsiccates $(37.44 \%)$ were incorrectly identified, which was not the case in some other taxonomically highly complicated groups, for example Cerastium subsect. Cerastium (Niketić 2007). More recent morphological research on certain species from $V$. sect. Viola has pointed out to complexity of the entire group and defines new characters adequate 
for more precise identification of taxa (Danihelka et al. 2009). The studies have shown that some characters, treated as diagnostic characters in earlier taxonomic, floristic and monographic studies, actually have much lower statistical significance. Additionally, the widely accepted idea that $V$. odorata and $V$. suavis, unlike the species $V$. hirta, have strongly developed stolons was not supported, as it was revealed that a significant percentage of individuals in populations of $V$. odorata and $V$. suavis in fact lacks these organs. However, even the characters proven to be statistically significant were not 100-percent applicable as there is always smaller or greater overlap between related species pairs.

The challenge of high variability and difficulty in identification and revision is illustrated very well by the species $V$. pumila. The only proven literature record of this species is collected by Josif Pančić from vicinity of Brestovačka Banja (BEOU - Herb Panc). For the other literature sources it may be assumed that they are actually relate to the species $V$. stagnina. The same situation applies to literature sources on $V$. elatior (sub $V$. persicifolia Schreb.). On the other hand most literature records for $V$. elatior actually pertain to the species $V$. jordanii.

In the edition "Flora of SR Serbia" (Diklić 1972) presence of 16 species from $V$. sect. Viola was cited for territory of Serbia. The results have shown that present flora of Serbia includes 14 out of these 16 species. As mentioned, the analysis included survey of two herbarium collections (BEOU and BEO), without consideration of literature data. Therefore presence of two species listed in the edition "Flora of SR Serbia" ( $V$. uliginosa and $V$. pyrenaica) could not be proven by the revision of the herbarium material in collections BEO and BEOU. It should also be noted that the edition "Flora of SR Serbia" (Diklić 1972) does not include species $V$. obliqua Hill., which may appear subspontaneously as a cultivated species in towns in Serbia. First records of this species were published by Lakušić et al. (2006).

The results of survey and revision of specimens from the herbarium collections BEOU and BEO have shown that $V$. sect. Melanium is represented in Serbia by 11 species. Species $V$. speciosa Pant., listed in the edition "Flora of SR Serbia" (Diklić 1972), is according to more recent conception of Erben (1985) considered a synonym for species $V$. elegantula Schott.

The greatest variability of morphological characters was recorded in species from group $V$. tricolor (species $V$. arvensis, $V$. kitaibeliana, $V$. tricolor s. 1.). Particularly troublesome was species $V$. tricolor $\mathrm{s}$. 1 . as three subspecies have been cited for flora of Serbia: $V$. $t$. subsp. tricolor, $V . t$. subsp. macedonica (Boiss. \& Heldr.) Schmidt and V. t. subsp. subalpina 
Gaud. (Diklić 1972). The type subspecies is most frequently an annual plant, mostly appearing in cultivated habitats, while other two subspecies are generally perennial plants connected with subalpine and alpine belts in Serbia (Diklić 1972, 1986). The incomplete herbarium specimens lacking the plant parts immediately above ground complicated determination whether the specimens came from annual or perennial plants, which is the key character for separation of these subspecies. Possibility of hybridization and high level of variability in morphological characters made the precise identification even more difficult. Additionally, some of the herbarium specimens collected in northeastern Serbia had morphological characters not matching any of the three listed subspecies. In many cases these specimens were first determined as the taxon $V$. banatica Kit., which is considered an infraspecies taxon of species $V$. arvensis under the contemporary classification systems, while according to results of our review it also shows some characteristics of $V$. tricolor. The high number of herbarium exsiccates of $V$. tricolor in the studied collections (506 sheets) actually supports the observation that this broadly defined species was an object of interest for numerous botanists and that it definitively includes several infraspecies taxa. This taxon certainly deserves more attention and additional field research, which would help solving the taxonomic issues within this interesting group.

There are also some challenges in identification and separation of the taxa $V$. elegantula and $V$. latisepala Wettst. According to Flora Europaea (Valentine et al. 1968), taxon $V$. latisepala Wettst. was considered as synonym for $V$. elegantula, Diklić (1972) treated it as var. latisepala (Wettst.) Beck of the same species, while Erben (1985) considered it as separate species. In this paper we followed the concept of Valentine et al. (1968) and included $V$. latisepala into $V$. elegantula. However, the problem with these two taxa is presently the subject of a detailed research, and the results will be published in near future.

Merxmüller (1974) emphasized that the proper identification of taxa of the $V$. sect. Melanium is coped with enormous difficulties and that it is as yet unachievable to conclude on the taxonomic relationship of all violets within the $V$. sect. Melanium simply by using only morphological characters. According to Schmidt (1963) acceptable identification demands experience due to the minor morphological differences of the Viola taxa on the Balkans. Erben (1985) stressed that one single character, allowing one to differentiate species quickly and with safety, does not exist for the related taxa within the $V$. sect. Melanium. For that reason, the analysis of a greater number of morphological characters is crucial. Still, when one is accustomed with the combination of the characters of different species, then their value is apparent even in the case of critical taxa (Erben 1985). 


\section{Acknowledgements}

The authors would like to thank colleague Vladan Đorđević who loaned us herbarium specimens of several Viola species. The Ministry of Education, Science and Technological Development of the Republic of Serbia supported this research through Grant 173030 "Plant biodiversity of Serbia and the Balkans - assessment, sustainable use and protection".

\section{REFERENCES}

Ballard, H. E,. Sytsma, K. J., Kowal, R. R. (1999): Shrinking the Violets: Phylogenetic Relationships of Infrageneric Groups in Viola (Violaceae) Based on Internal Transcribed Spacer DNA Sequences. - Systematic Botany 23(4): 439-458.

Clausen, J. (1964): Cytotaxonomy and distributional ecology of western North American violets. - Madroño 17: 173-204.

Danihelka, J., Niklfeld, H., Šípošová, H. (2009): Viola elatior, V. pumila and V. stagnina in Austria, Czechia and Slovakia: a story of decline. -Preslia 81: 151-171.

Delipavlov, D. (1979): Violaceae Batsch. In: Jordanov, D. (ed.): Flora na Narodna Republika B'lgaria 7: 338-395. - B'lgarskata Akademija na Naukite, Sofija.

Diklić, N. (1972): Violaceae. In: Josifović, M. (ed.): Flora SR Srbije 3: 128-164. Srpska Akademija nauka i umetnosti, Odeljenje prirodno-matematičkih nauka, Beograd.

Diklić, N. (1977): Violaceae. In: Josifović, M. (ed.): Flora SR Srbije 9: 72. Srpska Akademija nauka i umetnosti, Odeljenje prirodno-matematičkih nauka, Beograd.

Diklić, N. (1986): Violaceae. In: Josifović, M. (ed.): Flora SR Srbije 10: 59. Srpska Akademija nauka i umetnosti, Odeljenje prirodno-matematičkih nauka, Beograd.

Erben, M. (1985): Cytotaxonomische Unterschungen an Sudosteuropaischen Viola-arten der sektion Melanium. - Mitteilungen der botanischen Staatssammlung München 21: 339-740.

Grințescu, G., Guşuleac, M., Nyárády, E. I. (1955): Violaceae DC. In: Săvulescu, T. (ed.): Flora Republicii Populare Române 3: 553-625. - Academia Republicii Populare Române, Bucuresti.

Lakušić, D., Stevanović, V., Jovanović, S., Tomović, G. (2006): Reports 54-58. In: Vladimirov, V., Dane, F., Nikolić, T., Stevanović, V., Tan, K. (eds): New floristic records in the Balkans 2. - Phytologia Balcanica 12(2): 288-289. 
Lampinen R. (2001): Universal Transverse Mercator (UTM) and Military Grid Reference System (MGRS). [Available at: http://www.luomus.fi/english/botany/afe/map/utm.htm]

Mereda, P. Jr., Hodálová, I., Mártonfi, P., Kučera, J., Lihová, J. (2008): Intraspecific variation in Viola suavis in Europe: parallel evolution of white-flowered morphotypes. - Annals of Botany 102: 443-462.

Mereda, P. Jr., Hodálová, I., Kučera, J., Zozomova-Lihová, J., Letz, D. R., Slovák, M. (2011): Genetic and morphological variation in Viola suavis s.l. (Violaceae) in the western Balkan Peninsula: two endemic subspecies revealed. Systematics and Biodiversity 9(3): 211-231.

Merxmüller, H. (1974): Veilchenstudien I-IV. - Phyton (Austria) 16: 137-158.

Micevski, K. (1995): The Flora of the Republic of Macedonia I(3). - Macedonian Academy of Sciences and Arts, Skopje.

Niketić, M. (2007): Endemični predstavnici roda Cerastium L. u jugoistočnoj Evropi. Taksonomija, horologija i ekologija. Doktorska disertacija. - Biološki fakultet, Univerzitet u Beogradu.

Raus, Th. (1986): Violaceae. In Strid, A. (ed.): Mountain Flora of Greece 1: 608-640. - Cambridge University Press, Cambridge.

Schmidt, A. (1963): Zytotaxonomische Untersuchungen an griechischen Viola-Arten der Sektion Melanium. - Österreichische botanische Zeitschrift 110: 285-293 .

Słomka, A., Godzik, B., Szarek-Łukaszewska, G., Shuka, L., Hoef-Emdend, K., Bothe, H. (2015): Albanian violets of the section Melanium, their morphological variability, genetic similarity and their adaptations to serpentine or chalk soils. - Journal of Plant Physiology 174: 110-123.

Stevanović, V., Niketić, M. (1990): Viola dukadjinica W. Becker \& Košanin - a new species of the Yugoslav flora. - Slovenska Akademija Znanosti in Umetnosti. Razred za Naravoslovne Vede. Razprave 31: 317-326.

The Plant List 2013. Version 1.1. [http://www.theplantlist.org/] (accessed January $1^{\text {st }}$ )

Thiers, B. (2014): [continuously updated]. Index Herbariorum: A global directory of public herbaria and associated staff. New York Botanical Garden's Virtual Herbarium. [http://sweetgum.nybg.org/ih]

Valentine, D. H., Merxmüller, H., Schmidt, A. (1968): Viola L. In: Tutin, T. G., Heywood, V. H., Burges, N. A., Moore, D. M., Valentine, D. H., Walters, S. M., Webb, D. A. (eds): Flora Europaea 2: 270-282. - University Press, Cambridge. 


\title{
РОД Viola L. (ViOlaCEAE) У СРБИЈИ \\ - У ЗБИРКАМА ПРИРОДЊАЧКОГ МУЗЕЈАУ БЕОГРАДУ И \\ ИНСТИТУТА ЗА БОТАНИКУ И БОТАНИЧКЕ БАШТЕ „ЈЕВРЕМОВАЦ“, БИОЛОШКОГ ФАКУЛТЕТА, УНИВЕРЗИТЕТА У БЕОГРАДУ -

\author{
РАДОВИЋ, ГОРДАНА ТОМОВИЋ
} \\ МАРЈАН НИКЕТИЋ, СНЕЖАНА ВУКОЈИЧИЋ, КСЕНИЈА МИРЈАЧИЋ, АНА
}

\begin{abstract}
Р Е 3 И М Е
У раду је приказан каталог хербарских примерака за 27 врста/подврста из рода Viola L. са подручја Србије депонованих у две колекције: Хербаријуму Природњачког музеја у Београду (БЕО) и Хербаријуму Института за ботанику и Ботаничке баште "Јевремовац", Универзитета у Београду (БЕОУ). Прегледано је укупно 1936 хербаријумских табака од којих је 902 примерка депоновано у БЕО, а 1034 ексиката у БЕОУ. Поред тога, дате су карте распрострањења за сваку испитивану врсту/подврсту у Србији.
\end{abstract}

